\title{
Ultrasound Based Skeletal Motion Capture
}

the Development and Validation of a Non-Invasive Knee Joint Motion Tracking Method

\section{Kenan Niu}

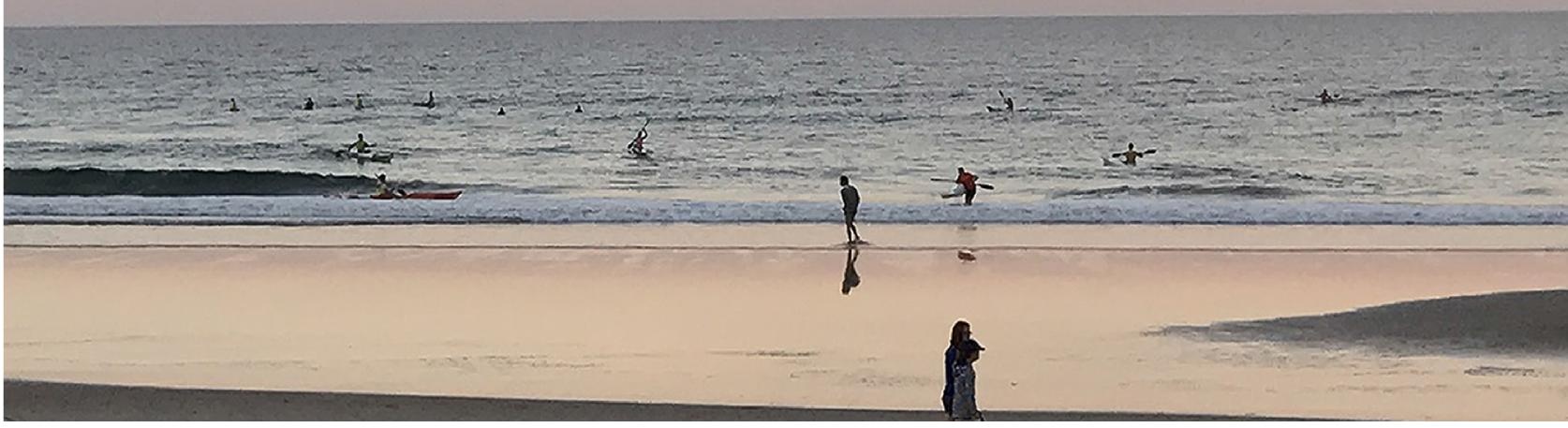





\section{Ultrasound Based Skeletal Motion Capture}

the Development and Validation of a Non-Invasive Knee

Joint Motion Tracking Method

Kenan Niu 
Composition of the graduation committee:

Chairman and secretary

prof. dr. G.P.M.R. Dewulf

University of Twente

Supervisor

prof. dr. ir. N.J.J. Verdonschot

University of Twente

Co-supervisors

dr. ir. J.J. Homminga

University of Twente

dr. A.M.J. Sprengers

Radboud University Medical Center

\section{Members}

prof. dr. ir. P.H. Veltink

University of Twente

prof. dr. S. Manohar

prof. dr. ir. G. J. Strijkers

University of Twente

prof. dr. B.M. ter Haar Romeny

Academic Medical Center Amsterdam

dr. M.M. van de Krogt

University of Technology Eindhoven

dr. Ing. S. van de Groes

VU University Medical Center

Radboud University Medical Center

Paranymphs

Victor Sluiter

Hao Chen

The work presented in this dissertation was conducted at the Laboratory of Biomechanical Engineering of the University of Twente, and carried out within the BioMechTools project. The research leading to these results has received funding from the European Research Council under the European Union's Seventh Framework Programme (FP/2007-2013)/ERC Grant Agreement n.323091.
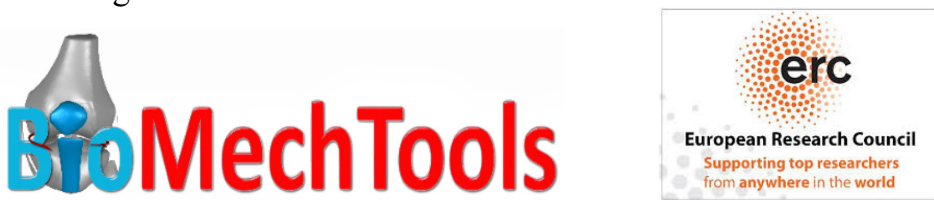

Financial support for the publication of this dissertation by the Laboratory of Biomechanical Engineering of the University of Twente is gratefully acknowledged.

Printed by Gildeprint

Cover design: Kenan Niu

ISBN: 978-90-365-4482-5

DOI: $10.3990 / 1.9789036544825$

URL: https://doi.org/10.3990/1.9789036544825

(C) Kenan Niu, Enschede, The Netherlands, 2018

All right reserved. No part of this publication may be reproduced, stored in an information storage or retrieval system, or transmitted in any form or by any means, electronic, mechanical, photocopying, recording, or otherwise, without the prior written permission of the holder of the copyright. 


\title{
ULTRASOUND BASED SKELETAL MOTION CAPTURE THE DEVELOPMENT AND VALIDATION OF A NON- INVASIVE KNEE JOINT MOTION TRACKING METHOD
}

\author{
DISSERTATION \\ to obtain \\ the degree of doctor at the University of Twente, \\ on the authority of the rector magnificus, \\ Prof. dr. T.T.M. Palstra, \\ on account of the decision of the graduation committee, \\ to be publicly defended \\ on Thursday $15^{\text {th }}$ February 2018 at 12.45
}

by

Kenan Niu

born on $18^{\text {th }}$ December 1988

in Laiwu, China 
This dissertation has been approved by the supervisor

prof. dr. ir. N.J.J. Verdonschot

and by the co-supervisors

dr. ir. J.J. Homminga

dr. A.M.J. Sprengers 


\section{Contents}

Contents 7

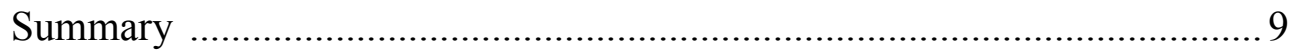

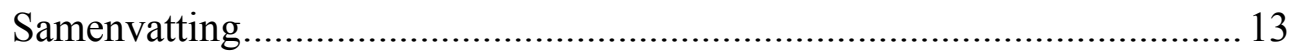

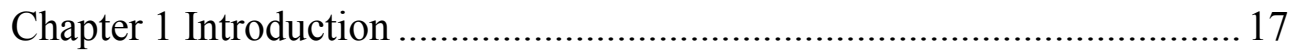

Chapter 2 Feasibility of A-mode ultrasound based intraoperative registration in computer-aided orthopedic surgery: a simulation and experimental study

Chapter 3 Measuring Tibiofemoral Kinematics Using One-channel 3D-

Tracked A-Mode Ultrasound Tracking System: A Proof of

Principle Study. .53

Chapter 4 A Novel Multi-channel 3D-Tracked A-Mode Ultrasound System

to Measure Tibiofemoral Kinematics 69

Chapter 5 In situ comparison of A-mode ultrasound tracking system and skin-mounted markers for measuring kinematics of the lower extremity

Chapter 6 A novel ultrasound tracking system to track in-vivo knee joint motions during walking and stair descent: a feasibility study .. 103

Chapter 7 General discussion and conclusions 119

Chapter 8 Supplementary technical details 129

List of Publications 155

Acknowledgement 157

About the author. 161 



\section{Summary}

Musculoskeletal disorders, particularly in the lower limb, are the most common cause of severe long-term pain and physical disability, and affect hundreds of millions of people around the world. Accurate measurement tools are required to diagnose these pathologies and to evaluate the efficacy of various treatment options. In this respect, detailed measurement and analysis of human movement have shown to be of great value.

Joint kinematic data can be used in motion analyses combined with biomechanical modelling, e.g. musculoskeletal models for inverse dynamics approaches. Subsequently, researchers can utilize these musculoskeletal models to better understand the dysfunctions of the musculoskeletal system, and try to improve the diagnosis and treatment for patients. Hence, as a critical input to the musculoskeletal models, a valid representation of actual skeletal motion and kinematics are of high relevance. However, the fact is that human skeletal structures are not exposed to the outside environment but are surrounded by soft tissues (muscles, fat, skin etc.). Therefore, an effective measuring technique that could directly or indirectly detect the motion of the bony segments is necessary to quantify the movements of these segments inside the body.

To measure skeletal kinematics of the lower extremity researchers have previously used intra-cortical bone pins with mounted markers. The positions of the inserted bone pins were measured by a stereo photographic system (e.g. optical tracking system). It has been demonstrated that this method provides a very accurate representation of the motion of bone segments in the knee joint. However, the invasiveness of this method impedes its application into clinical practice. As an alternative, utilization of skin-mounted markers is currently the most widely used method for measuring kinematics of the lower extremity for gait analysis, in which the trajectories of skin-mounted markers represent the movements of the bony segments beneath the skin. However, this method is limited by its accuracy of estimated kinematics, which is subject to Soft Tissue artifacts (STA). Alternatively, fluoroscopic systems utilize radiographic images and adequate model based methods to be able to achieve high accuracy, but induced irradiation to the subject and a limited field of view hamper routine usage in the clinical setting.

Ultrasound (US) provides the possibility of detecting the tissue-bone boundary and estimating its depth through the soft tissue during movement with the advantages of noninvasiveness and non-radiation. The goal of this research was to develop and validate a new non-invasive method based on ultrasound that is able to track skeletal motion around the knee joint and to quantify tibiofemoral kinematics under dynamic conditions. To achieve this goal 


\section{Summary}

we separated the work in various parts which are outlined in this thesis and which are summarized below:

Firstly, to investigate the feasibility of point cloud registration algorithm implementation for this specific application, a numerical simulation was conducted to explore the possibility of registering the detected point cloud with a known bone shape model in Chapter 2 . Furthermore, the sensitivity to the number of acquired points and the sensitivity to induced noise were also investigated. A cadaveric experiment was also conducted to evaluate the registration method that is capable of determining the $3 \mathrm{D}$ position of a bone segment at a fixed position. The results showed that our registration method (ICP with Perturbation Search) had a significant improvement on the registration accuracy relative to the standard ICP registration method. The results of Chapter 2 furthermore demonstrated the possibility of registering a known bone shape to a sparse point cloud and provided a guideline to decide the necessary number of A-mode ultrasound transducers that should be used in the first prototype system. We found that 15 points could get an acceptable accuracy (Root Mean Square Errors, RMSE $<1.5 \mathrm{~mm}$ ) when localization error is low $(<1 \mathrm{~mm})$. Hence, we decided to employ 15 A-mode ultrasound transducers for each bone segment. Thus, based on this study, a total number of 30 A-mode ultrasound transducers was selected to be used for our final system.

Secondly, the principle of digitizing a bony point from a tracked A-mode ultrasound probe was investigated in a cadaveric setting in Chapter 3. The skeletal positions of shank and thigh were estimated while fixing the bones in different stationary positions. We assumed that measuring multiple bony points covering the shank and thigh at one stationary position would provide a data set that would be comparable to the simultaneous measurement of bony points by multiple tracked A-mode ultrasound probes. Under these circumstances, the obtained bony points at one stationary knee position were processed to quantify tibiofemoral relative positions and orientations between the femur and tibia in a static manner. The derived rotational and translational outcomes were compared to the reference outcomes that were derived from inserted intra-cortical bone pins that we considered as the ground truth. The results derived from the tracked A-mode ultrasound probe achieved a mean of $1.06 \pm 2.05^{\circ}$ and $-2.16 \pm 3.02 \mathrm{~mm}$ error for rotational components and translational components, respectively, which showed a relative high accuracy in estimated rotations and translations. The results of this method showed the potential to estimate tibiofemoral kinematics under a dynamic situation when simultaneously tracking an array of A-mode transducers covering the lower extremity at multiple locations. Hence, after this feasibility study it was decided to acquire the hardware to build a multi-channel A-mode system. 
Thirdly, we developed a multi-channel A-mode ultrasound tracking system as described in Chapter 4. The unique feature of our proposed ultrasound tracking system lies on the extension of the detection range of skin-mounted markers from the superficial measurement to the real bony surface. The combination of multiple A-mode ultrasound transducers with a conventional motion capture system improves the validity of the estimated 3D positions of the underlying bony segments which are required to accurately quantify tibiofemoral kinematics in a dynamic fashion. The results of Chapter 4 show the accuracy of the estimation in flexion-extension (RMSE $1.51^{\circ}$ ), adduction-abduction (RMSE $0.88^{\circ}$ ). Average rotational errors of $1.51 \pm 1.13^{\circ}$ (mean $\pm \mathrm{SD}$ ) and average translational errors of $3.14 \pm 1.72 \mathrm{~mm}$ (mean $\pm \mathrm{SD}$ ) were obtained. Although the reconstructed tibiofemoral kinematics were less accurate than those reported for fluoroscopic systems, it has the potential to overcome the effects of soft tissue artifacts of skin-mounted markers systems to produce accurate kinematics.

Subsequently, an in-situ comparison was conducted in a cadaveric experiment in order to investigate the performance of our method against the more traditional method using skinmounted markers with the constrained hinge and spherical knee models (in Chapter 5). The ultrasound tracking system resulted in lower kinematic errors than the skin-mounted markers (the ultrasound tracking system: maximum RMSE $3.44^{\circ}$ for rotations and $4.88 \mathrm{~mm}$ for translations, skin-mounted markers with the spherical joint model: $6.32^{\circ}$ and $6.26 \mathrm{~mm}$, the hinge model: $6.38^{\circ}$ and $6.52 \mathrm{~mm}$ ). The ultrasound tracking method resulted in lower kinematic errors, in the experimental conditions investigated, and could represent a viable alternative to traditional system, which could improve the measurement accuracy of bone and joint kinematics.

Eventually, we aimed to demonstrate the feasibility of capturing skeletal motions and quantifying tibiofemoral kinematics for healthy subjects performing different daily activities (in Chapter 6). After changing several gait parameters, e.g. imposed speed of treadmill and height of staircase, kinematic alterations could be quantified which were in accordance with previous findings. Although it should be realized that there was no ground truth measurement accompanied in this experiment, the experiment was valuable in demonstrating some features of the system that are important for in-vivo assessment of human movement, such as walking and stair descent. Furthermore, we were successful in obtaining on-line (real time) kinematic data, which is important to directly assess the quality of the measurement. Finally, we demonstrated that the system prototype worked appropriately under in-vivo conditions and therefore has the potential to measure skeletal motion in research and clinical applications.

In conclusion, ultrasound based skeletal motion capture is feasible and has the potential to achieve high accuracy in the estimation of skeletal motion and quantification of 6-DOF joint kinematics. The currently developed system showed the ability to directly measure skeletal 


\section{Summary}

kinematics despite soft tissue deformations between the transducer and the bony surface. Therefore, this method has great potential to be considered as a suitable alternative for measuring human skeletal motion.

In this thesis a considerable number of improvement steps are described which will enable to achieve higher accuracies and sampling rates than those described in this thesis. After implementation of these improvements a unique measurement system can be obtained that can be applied to a variety of applications such as quantification of dynamic motion and deformation of soft tissue structures, general gait analysis, prosthetic design optimization, orthopaedic reconstructive surgery and surgical navigation. This thesis provides the foundation for these future applications. 


\section{Samenvatting}

Aandoeningen aan het spierskeletsysteem, met name in de onderste extremiteit, zijn de meest voorkomende oorzaken van ernstige chronische pijn en fysieke beperkingen en komt wereldwijd voor bij honderden miljoenen mensen. Een nauwkeurige bepaling van de beweging van de onderste extremiteit is een vereiste voor een correcte diagnose en een optimale behandeling. Gedetailleerde meting en analyse van het menselijk bewegingsapparaat zijn in deze context van grote waarde gebleken.

Gewrichtskinematica kan worden gebruikt in combinatie met biomechanische modellen zoals spierskeletmodellen met inverse dynamica. Onderzoekers kunnen deze modellen gebruiken om afwijkende functionaliteit van het spierskeletsysteem te bestuderen en bij te dragen aan een verbeterde diagnose en behandeling van patiënten. De meerwaarde van een dergelijk model is in grote mate afhankelijk van een nauwkeurige meting van de daadwerkelijke kinematica van het skelet. Correcte meting van de beweging van de botsegmenten wordt echter bemoeilijkt door de omliggende zachte weefsels (spier, vet, huid, etc.). Een effectieve techniek zal derhalve op directe of indirecte wijze de beweging van de botsegmenten moeten kunnen volgen binnen het lichaam.

Eerder onderzoek heeft gebruik gemaakt van botpennen waarop markers bevestigd waren. De positie van de botpennen kon dan worden gevolgd met behulp van een stereo fotografisch systeem (b.v. een 3D optisch meetsysteeem). Deze benadering verschaft weliswaar een zeer nauwkeurige weergave van de beweging van de botten, maar de invasiviteit van de methode belemmert toepassing in de kliniek. Markers op de huid vormen een niet-invasief alternatief en worden op dit moment veelvuldig gebruikt in academische en klinische onderzoeken om skeletkinematica te meten. Markers worden op meerdere plekken op de huid aangebracht en representeren dan de positie van het bot vlak onder de huid. Op basis van beweging van de markers ten opzichte van elkaar en ten opzichte van de ruimte, kan de botkinematica worden gereconstrueerd. Deze methode is aanzienlijk minder invasief dan de methode met botpennen, maar boet dientengevolge in aan nauwkeurigheid. Deze verlaagde nauwkeurigheid wordt veroorzaakt door de beweging van de huid ten opzichte van het bot (zogenaamde Soft Tissue Artifacts; STA). Een derde benadering voor het meten van botkinematica is fluoroscopie. Fluoroscopische systemen maken gebruik van röntgenstraling om de botten in $3 \mathrm{D}$ te visualiseren tijdens beweging. Deze methode heeft een nauwkeurigheid die vergelijkbaar is met die van botpennen. Echter wordt deze methode veelal niet verkozen boven markers op de huid vanwege het gebruik van röntgenstraling en het beperkte zichtveld waarin botsegmenten kunnen worden gevisualiseerd. 


\section{Samenvatting}

Echografie ofwel 'ultrasound' is in staat het bot onder de huid te detecteren zonder gebruik van straling of een invasieve ingreep. Het doel van dit project was het ontwikkelen en valideren van een nieuwe niet-invasieve methode gebaseerd op ultrasound voor het meten van skeletbeweging rondom het kniegewricht en de kwantificatie van tibiofemorale kinematica met een nauwkeurigheid vergelijkbaar met die van botpennen of fluoroscopie met behoud van de klinische toepasbaarheid van markers op de huid. Voor het bereiken van dit doel zijn meerdere studies verricht die als volgt zijn beschreven in dit proefschrift:

Allereerst is een studie gedaan om de haalbaarheid te demonstreren van het bepalen van $3 \mathrm{D}$ oriëntatie en positie van botmodellen op basis van puntenwolken (hoofdstuk 2). In deze studie is de gevoeligheid van de registratiemethode voor het aantal gemeten punten en de ruis (fouten) op deze punten onderzocht. De numerieke methode is onderbouwd met een kadaverexperiment door in het experiment te toetsen in hoeverre het mogelijk is om de $3 \mathrm{D}$ positie van een bot te reconstrueren is op basis van slechts een beperkt aantal gemeten punten op het bot. Uit de resultaten bleek dat de gebruikte registratie methode (Iterative Closest Points (ICP) met perturbatie) een significante verbetering oplevert in nauwkeurigheid van registratie versus de standaard ICP methode. Daarnaast toonden de resultaten aan dat het fundamenteel mogelijk is een botmodel te registreren op basis van een klein aantal gemeten punten op het bot en ze gaven een indicatie voor het benodigde aantal A-mode ultrasound transducers voor het behalen van een bepaalde vereiste nauwkeurigheid. Op basis van deze indicatie is er gekozen voor het gebruik van 15 transducers per bot segment (30 voor de combinatie van femur en tibia) voor het uiteindelijke systeem voor het behalen van een acceptabele foutmarge (RMS fout $<1.5 \mathrm{~mm}$ gegeven een lokalisatiefout $<1 \mathrm{~mm}$ ).

In hoofdstuk 3 wordt, in een kadaver experiment, het principe van het verkrijgen van een de locatie op een bot met behulp van een in 3D gevolgde A-mode ultrasound sensor onderzocht. De posities van het onder- en bovenbeen zijn gemeten terwijl de botten in meerdere stationaire houdingen gefixeerd waren. We namen hierbij aan dat het sequentieel meten van meerdere botpunten verdeeld over onder- en bovenbeen in een gefixeerde houding een dataset zou opleveren die vergelijkbaar is met een meting die gebruik maakt van meerdere A-mode sensoren die simultaan bemeten worden. De gemeten botlokaties per stationaire houding zijn vervolgens verwerkt om tibiofemorale relatieve posities en orientaties te kwantificeren. De berekende rotaties en translaties zijn vervolgens vergeleken met data van intra-corticale botpennen die we als basisreferentie beschouwden. Deze vergelijking toonde aan dat de A-mode ultrasound sensor een gemiddelde fout van $1.06 \pm$ $2.05^{\circ}$ voor rotaties en $-2.16 \pm 3.02 \mathrm{~mm}$ voor translaties heeft. Dit geeft een indicatie voor de relatief hoge nauwkeurigheid van gemeten rotaties en translaties bij gebruik van deze methode. De resultaten gaven daarmee vertrouwen in de potentie van een systeem van 
meerdere A-mode transducers, geplaatst op de huid op meerdere locaties verdeeld over de onderste extremiteit, voor het nauwkeurig schatten van tibiofemorale kinematica onder statische omstandigheden. Op basis van deze haalbaarheidsstudie is besloten hardware te kopen voor het bouwen van een meerkanaals A-mode systeem.

In hoofdstuk 4 wordt de ontwikkeling van het meerkanaals A-mode ultrasound 3Dmeetsysteem beschreven. Het unieke kenmerk van dit systeem ligt in de uitbreiding van het meten van huidlocaties met een marker systeem naar het meten van botpunten vlak onder de huid middels ultrasound. De combinatie van meerdere A-mode ultrasound sensoren met een conventioneel 3D-meetsysteem biedt een hogere 3D positie nauwkeurigheid van botsegmenten. Met behulp van deze positie data kan tibiofemorale kinematica worden gereconstrueerd onder dynamische situaties. Resultaten uit hoofdstuk 4 lieten de nauwkeurigheid in flexie-extensie (RMS fout $1.51^{\circ}$ ), en adductie-abductie (RMS fout $0.88^{\circ}$ ) zien. Het gemiddelde van de fout in rotaties is $1.51 \pm 1.13^{\circ}$ (gemiddelde \pm SD) en de gemiddelde fout in translaties is $3.14 \pm 1.72 \mathrm{~mm}$ (gemiddelde $\pm \mathrm{SD}$ ). De gemeten tibiofemorale kinematica had een lagere nauwkeurigheid dan de nauwkeurigheid die gerapporteerd is bij fluoroscopie. Het systeem toont echter de potentie om kinematica te meten met minder verstoring door effecten van zacht weefsel dan bij een conventioneel systeem met markers op de huid.

Hoofdstuk 5 beschrijft een kadaverexperiment waarbij een in-situ vergelijking werd onderzocht van de methode ontwikkeld in dit proefschrift met de conventionele skin marker methode (toegepast met zowel een scharnier als een sferisch kniemodel). Met behulp van het ultrasound tracking systeem zijn we er in geslaagd een hogere nauwkeurigheid te behalen dan het skin marker systeem: een maximum RMS fout van $3.44^{\circ}$ voor rotaties en $4.88 \mathrm{~mm}$ voor translaties met het ultrasound systeem; $6.32^{\circ}$ en $6.26 \mathrm{~mm}$ voor het skin marker systeem gereconstrueerd met een sferisch model en $6.38^{\circ}$ en $6.52 \mathrm{~mm}$ voor het skin marker systeem gereconstrueerd met het scharnier model. Onder de condities van dit experiment levert de ultrasound methode een hogere nauwkeurigheid en vormt daarmee een mogelijk alternatief voor het meten van kinematica in het knie gewricht, met een hogere nauwkeurigheid en gelijkwaardige patiëntvriendelijkheid.

Hoofdstuk 6 beschrijft een haalbaarheidsstudie voor het toepassen van het ontwikkelde ultrasound systeem voor het meten van kinematica in gezonde vrijwilligers tijdens het uitvoeren van dagelijkse bewegingen. Veranderingen in de kinematica aan de hand van veranderingen in meerdere parameters zoals snelheid van een loopband, en hoogte van een traptrede zijn gekwantificeerd en vergeleken met resultaten uit de literatuur. Alhoewel er in dit experiment niet vergeleken kon worden met een zogenaamde 'ground truth', is met deze resultaten wel gedemonstreerd dat het systeem bruikbaar is voor metingen van belangrijke 


\section{Samenvatting}

parameters van gangbare bewegingen, zoals wandelen en traplopen. Daarnaast hebben we gedemonstreerd dat het met dit systeem mogelijk is om real-time kinematische data te meten, zodat de data direct kunnen worden bestudeerd. Tot slot hebben we kunnen demonstreren dat het met dit prototype systeem mogelijk is om kinematische metingen te doen bij gezonde vrijwilligers onder omstandigheden die klinisch toepasbaar zijn.

Concluderend beschrijven we in dit proefschrift een systeem voor het meten van skeletbeweging in 6 graden van vrijheid in de onderste extremiteit gebaseerd op ultrasound, met de potentie van een aanzienlijk hogere nauwkeurigheid, vergeleken met conventionele skin marker methodes. Het systeem is in staat om beweging tussen bot en huid te ondervangen en de beweging van het bot direct te volgen. Deze methode is derhalve een goed alternatief voor het meten van skeletbeweging met skin markers.

In dit proefschrift zijn meerdere mogelijkheden beschreven om het huidige prototype te verbeteren en tot een hogere nauwkeurigheid en bemonsteringsfrequenties te komen dan wat nu beschreven is in de experimenten in dit proefschrift. Implementaties van deze verbeteringen zullen leiden tot een uniek systeem geschikt voor het meten van hoogdynamische beweging, complexe vervorming van de zachte weefsels, standaard looppatroonanalyse, optimalisering van prothese ontwerp en planning en navigatie van orthopedische chirurgie. Dit proefschrift geeft een basis voor de ontwikkeling van deze toepassingen. 
Introduction

Chapter 1 Introduction 


\subsection{Background}

Most animals are capable of moving, with few exceptions that do not move their whole bodies, or relocate to new positions (like coral and sea anemones). Human beings, as Vertebrates, are not like Arthropods (crab, spider and centipede) having an exoskeleton (external skeleton) or Molluscs (snail, octopus and slug) having no skeletal structure. For humans, the skeletal structures are surrounded by soft tissues, such as muscle, fat, tendons, skin, etc. The neural system controls the combination of muscles to generate forces in order to move the skeleton for different tasks. Thus activities such as walking, sitting down, standing up, climbing, squatting, and dancing, can be performed easily and naturally in our daily life. However, musculoskeletal disorders influence many different parts of the body, including neck, shoulders, back, upper extremities (hand, wrist, arm, elbow), and lower extremities (hip, knee, ankle, feet) and subsequently may limit the execution of daily activities and reduce the quality of life. It is important to determine how these activities are affected by musculoskeletal disorders and to diagnose the pathological changes occurring at the joint and within the limb as a whole [1]. Furthermore, during orthopedic surgery, accurate three-dimensional (3D) positions and orientations of the bone segments are required to correct for limb malalignment or to accurately position prosthetic components e.g. during total knee arthroplasty (TKA) [2, 3] and total hip arthroplasty (THA) [4].

However, accurate quantification of bone positions and human movement parameters is quite challenging. This is because observing and describing the subtle skeletal motions of humans is a non-trivial task, particularly under dynamic conditions, since the direct observation of skeletal motion of humans is impossible in practice without an auxiliary tool which helps to 'view' the skeleton beneath the outer surface of the body. The research as described within this thesis is concentrated on the knee joint, especially the tibiofemoral joint.

\subsection{Requirements}

To know the positions of the bony segments around the knee, an accurate method that is capable of measuring skeletal motion of the knee joint and quantifying six-degree-of-freedom (6-DOF) tibiofemoral kinematics in a non-invasive and non-radiative manner would be a desirable. The required accuracy of kinematic measurement for tibiofemoral joint differs depending on the clinical application:

- Gait analysis: $2^{\circ}$ and $2 \mathrm{~mm}[5]$.

- Prosthetic kinematics: $1^{\circ}$ and $1 \mathrm{~mm}[6-8]$.

- Surgical navigation: $1^{\circ}$ and $1 \mathrm{~mm}$ or below $2 \mathrm{~mm}$ registration accuracy $[3,9]$. 


\subsection{Current techniques}

In order to capture the skeletal motion in the knee joint and to quantify the tibiofemoral kinematics under dynamic conditions, several techniques have been developed and applied for various clinical scenarios. These techniques are summarized in Figure 1.1.

\subsubsection{Intra-cortical bone pins}

The most direct approach to measure relative motion of two bony segments is to fixate tracked intra-cortical bone pins to the bone segments $[10,16]$. These intra-cortical bone pins remain in a fixed position relative to the corresponding bone segments. Hence, the generally made assumption that the relative positions between intra-cortical bone pins and bone segments are rigid can be considered as acceptable. The intra-cortical bone pins are typically tracked by motion capture systems or surgical navigation systems. This method provides an accurate measurement of the skeletal motion and is considered as the gold standard in tracking skeletal motion and quantifying skeletal kinematics. However, the invasiveness of the method impedes routine usage in most clinical scenarios, except during surgery.

\subsubsection{Skin markers}

Currently, skin-mounted markers are widely used in capturing human motion. The active or reflective markers attached on the skin are tracked by stereo photographic techniques, in which the trajectories of skin-mounted markers are recorded and represent the movement of the subject. When we then assume that the movement of the skin is rigid relative to the bone movement, this measurement represents the underlying skeletal motion. The deformation between the underlying skeletal structure and the superficial skin surface, however, results in inaccuracies in estimated skeletal motion which generally propagates to the kinematic estimation and contaminates their validity in representing underlying bone motion. It has been reported that Soft Tissue Artefacts (STA, the term generally used for these types of errors) can cause measurement errors of skin markers up to $30 \mathrm{~mm}$ in the thigh [17]. The propagation of STA to knee joint kinematics has been reported to lead to average rotational errors of up to $4.4^{\circ}$ and $13.1^{\circ}$ and average translational errors of up to $13.0 \mathrm{~mm}$ and $16.1 \mathrm{~mm}$ for walking and cutting motions, respectively [11].

Extensive research has been conducted on quantification, assessment, and compensation of STA for different motor tasks [18-23]. Multi-body Kinematics Optimization (MKO) has been used with the intent to compensate the STA and to limit the propagation of STA to joint kinematics estimation [19,23]. Unfortunately, MKO generally reduces the errors slightly but does not completely resolve the problems caused by STA $[5,24]$. The researchers stated that the motion analysis research community should make more efforts in searching of more 


\section{Introduction}

advanced subject-specific joint models or error models, or a new measurement modality in order to improve the accuracy of estimated joint kinematics [5, 24, 25].

\subsubsection{Fluoroscopic systems}

With the development of medical imaging technologies, fluoroscopic systems have been utilized to capture highly accurate skeletal motion and joint kinematics in the knee joint [6, $8,26]$. Fluoroscopic systems utilize radiographic images and adequate model based methods [6, 27-29] to be able to achieve an accuracy in the order of $1 \mathrm{~mm}$ for translations and 1 degree for rotations with the bi-planar fluoroscopic imaging system [30-32]. However, radiation exposure of patients, high cost, cumbersome setup, and limited field of view (FOV) impede routine usage in a clinical setting. Recently, several groups have been working on the development of mobile fluoroscopy systems [7, 31, 33]. Although using a robotic trolley or gantry that carries the fluoroscopic system while following the movement of a subject extends the FOV, the radiation exposure of the patient remains a problem. In addition, the workload, low availability and high cost still hinder its transfer from a laboratory setting to clinical routine usage.

\subsubsection{Roentgen stereophotogrammetric analyses}

Roentgen stereophotogrammetric analysis (RSA) is a radiographic technique used in the orthopedic field for measuring micro-motion at bone-prosthesis interface or for joint kinematics evaluation [34-36]. The RSA uses two X-ray sources synchronized with two digital flat-panels, which allows a quantitative evaluation of the joint kinematics during the recovery time [37]. However, similar to fluoroscopic systems, the radiation exposed to the subject cannot be avoided and the systems are normally cumbersome and it is difficult to capture dynamic motions.

\subsubsection{D dynamic MRI and CT}

Recently, advanced four-dimensional (termed 4D, including 3D spatial domain with time domain) MRI [15, 38, 39] and CT [12, 40] techniques have been used to track the bone motion and to quantify joint kinematics inside the scanners. The obvious advantage of these methods is that the actual 3-D kinematics of the joint can directly be extracted from the images. The disadvantages of these methods are the limited FOV, limited sample rate (4D $\mathrm{MRI}<1 \mathrm{~Hz}, 4 \mathrm{D} \mathrm{CT}: 10-20 \mathrm{~Hz}$ ) and the inability to measure kinematics during daily activities.

Similar to the fluoroscopic systems, 4D dynamic MRI and CT systems also have the drawbacks of high cost, high workload and limited availability. 


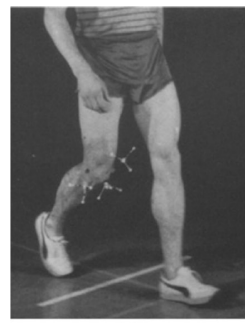

(a)

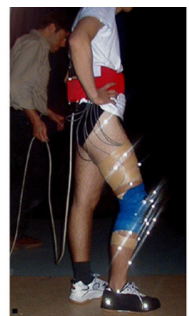

(b)

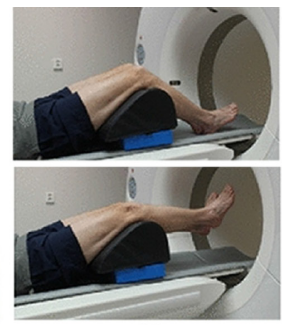

(c)

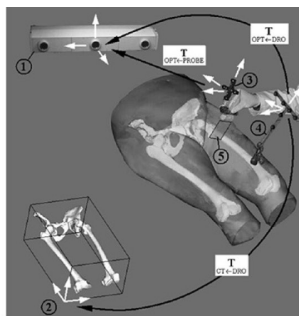

(d)

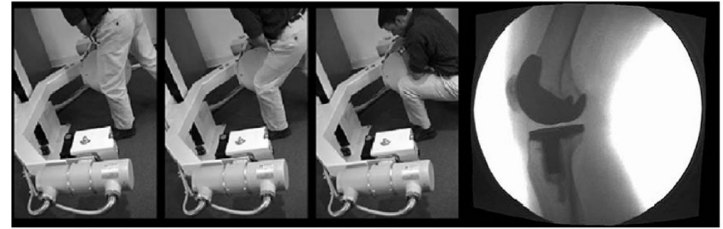

(e)

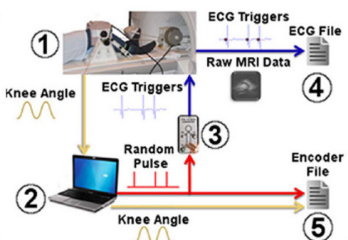

(f)

Figure 1.1 A schematic of several current techniques to capture skeletal motions and to quantify related kinematics. (a) intra-cortical bone pins were used to record the motions of the femur, tibia and patella. Reprinted from [10], Copyright (1992), with permission from Elsevier; (b) skin markers attached on the lower extremity were used in gait analysis. Reprinted from [11], Copyright (1992), with permission from Elsevier. (c) four-dimensional CT scan for knee joint. Reprinted from [12], Copyright (2016), with permission of Springer. (d) ultrasound combined with surgical navigation system for computer assisted orthopedic surgery. (C) [2006] IEEE: [13]. (e) fluoroscopic system and a radiographic image of in-vivo total knee replacement. (C) [2003] IEEE: [14]. (f) four-dimensional MRI scan for tracking knee motion. Reprinted from [15], Copyright (2013), with permission of WILEY.

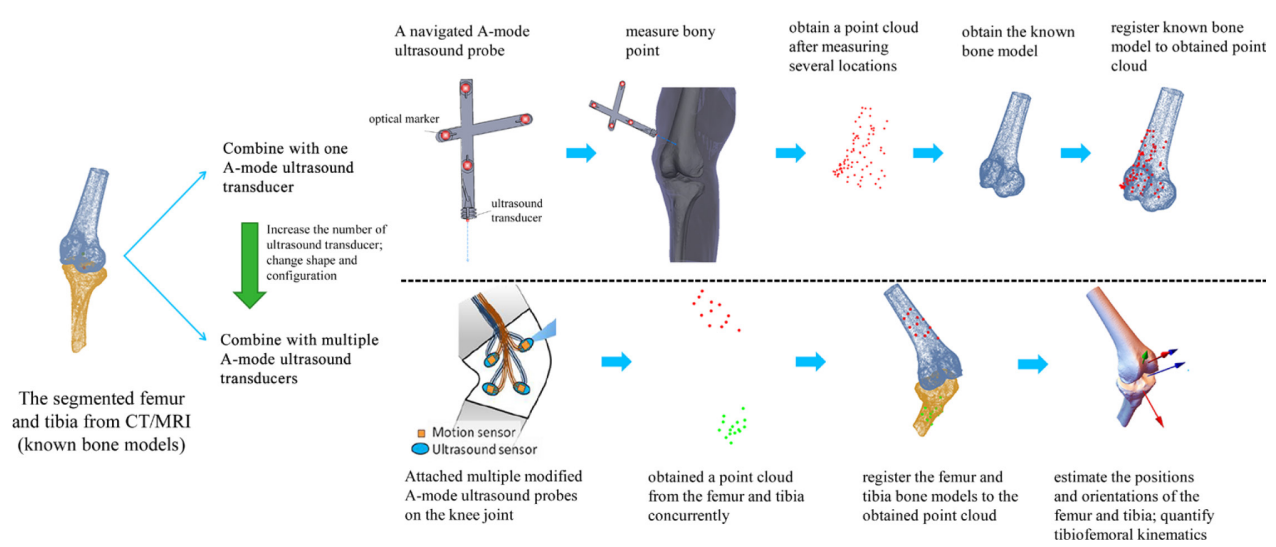

Figure 1.2 A schematic representation of proposed ultrasound based motion tracking system that extends the number of bony points acquired concurrently from a navigated A-mode ultrasound probe to multiple modified A-mode ultrasound probes. (Top): The schematic of a navigated A-mode ultrasound probe for bone registration; (bottom): our proposed ultrasound based motion tracking system by increasing the number of A-mode ultrasound transducers and change their shape and configuration in order to obtain a point cloud of the femur and tibia concurrently over a period of time. 


\subsubsection{Ultrasound}

Ultrasound techniques have been developed and applied in many clinical applications, particularly for assessments in soft tissues and internal organs [41-44]. Ultrasound imaging techniques offer a truly non-invasive and non-radiative method of evaluating internal anatomical structures. Since ultrasound is capable of visualizing the bone surface through the soft tissue, ultrasound-based intraoperative registration have been utilized to eliminate the need for physical contact with the bone surface $[45,46]$. It has been shown to be possible to register US images to segmented bone models in Computer-Aided Orthopedic Surgery (CAOS) within the, for example, knee, spine and hip regions [13, 47, 48].

Ultrasound transducers have been developed and utilized to measure bone surfaces. Amode ultrasound transducers (single element ultrasound transducers) have successfully been used for intraoperative registration in CAOS [44, 49-51]. The combination of a CAOS system and a single A-mode ultrasound transducer has been used in skull [52, 53], pelvic [54], and knee [51] surgery. An A-mode ultrasound transducer could be seen as a virtually extended measurement line from the skin to the bone surface. A navigated A-mode ultrasound transducer provides the possibility of measuring a 3D bony point through layers of soft tissue [51] (see Figure 1.2).

A navigated B-mode ultrasound transducer could then provide multiple digitalized 3D bony points after processing the acquired ultrasound image [13, 47]. The feasibility of estimating knee joint kinematics based on conventional B-mode ultrasound transducers has also been shown [55]. Consequently, researchers have proposed a similar concept by combining a B-mode ultrasound transducer with a motion capture system, which could obtain one or multiple curves representing bone surfaces $[55,56]$. The potential of compensating soft tissue artefacts with a B-mode transducer has been validated in an in-vivo experiment by measuring the depth of bone surfaces on the greater trochanter with up to $16 \mathrm{~mm}$ Euclidean distance difference between ultrasound measurement to skin marker measurement [56]. However, to our knowledge, dynamic kinematic measurements with ultrasound transducers with the goal of removing the soft tissue artifacts have not been performed or validated in previous studies.

\subsection{Ultrasound based motion tracking system}

All current techniques for skeletal motion capture and skeletal positioning have drawbacks involving one or more technical restrictions (limited FOV, low accuracy, and low frame rate), monetary restrictions (high cost and workload) and ethical restrictions (extra incision or radiation). A new technique without these drawbacks would be considered as a suitable 
alternative method that could be applied in a variety of clinical applications from surgical navigation (skeletal positioning) to human motion analysis (skeletal motion tracking) and post-operative evaluation (prosthetic kinematics).

In this project we set out to develop a new, non-invasive and non-radiative method to capture skeletal motions of the knee joint and to quantify tibiofemoral kinematics under dynamic conditions. Since ultrasound imaging is a suitable imaging modality to offer a noninvasive and non-radiative measurement, it can be considered as an appropriate candidate to detect the bone surface in $3 \mathrm{D}$. When considering the feasibilities in technical and practical aspects between A-mode and B-mode ultrasound transducers, the A-mode transducers have the advantage over B-mode transducers in terms of cost, size, availability and effectiveness in simultaneously using multiple-transducers to cover different anatomical areas. Compared to the conventional B-mode ultrasound transducers, A-mode ultrasound transducers are cheaper and appear to be more suitable for determining the bone surface in real-time [57, 58]. Its localization accuracy was reported to be approximately $0.4 \mathrm{~mm}$ after calibration $[49,51$, 59]. A combination of multiple A-mode ultrasound transducers and conventional skinmounted markers provides a new approach to measuring the trajectories of underlying bone segments in the thigh and shank. When a larger number of A-mode transducers is used and the configuration and placement of these A-mode transducers are tuned to fit the geometrical structure of anatomical areas on the lower extremity, multiple bony points (a point cloud) could be detected and obtained concurrently over a period of time (see Figure 1.2). If sufficient bony points are acquired from the surface of a known bone shape model, there would be a unique solution to match all bony points to the known bone shape model, which means that these discrete bony points could represent a unique 3D position and orientation of the known bone segment. In this context, for the next step, those bony points could be used to register known patient specific bone models (normally obtained from CT or MRI scans or bone morphing) and thus obtain the $3 \mathrm{D}$ positions and orientations of the bone segments of the subject during movement. In the knee joint, when the relative position of the femur with respect to the tibia is known, tibiofemoral kinematics can subsequently be quantified (see Figure 1.2).

To realize this concept towards a prototype or a product, several primary functional modules should be developed and evaluated (see Figure 1.3)

- Obtain a cloud of discrete points obtained from the bone surface (hardware acquisition)

- $\quad$ Register the known bone shapes to the cloud of discrete points (software processing)

- Derive joint kinematics from the registered bone shapes (outcomes determination) 


\section{Introduction}

Calculating joint kinematics from a known spatial relation between two bone segments is well defined [60], thus the core functional modules that need to be developed in this project are twofold: 1) the acquisition of sufficient bony points; 2) registering known bone shapes to the obtained bony points.

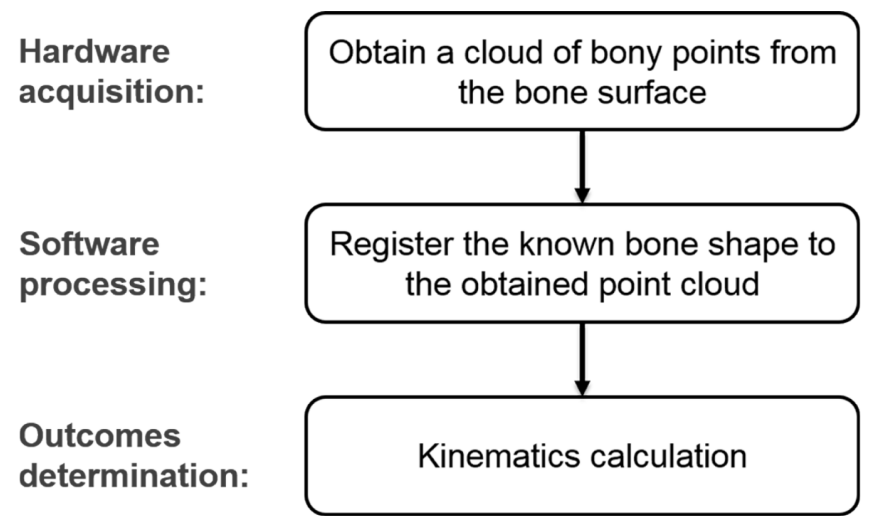

Figure 1.3 the conceptual workflow of ultrasound motion tracking system

To achieve the goal of this study and to assess the feasibility of this new method, several research questions need to be addressed step by step in the following chapters and will be critically assessed in the Discussion chapter.

Q1. How many points are needed to determine the 3D position of a bone segment using this method and with what accuracy can this be obtained? How do the localization errors of the points affect the accuracy of segment position?

Q2. Is this method capable of determining the 3D position of a bone segment at a fixed position and with what accuracy?

Q3. Is it possible to quantify tibiofemoral kinematics at different static positions using this method and with what accuracy?

Q4. Is this method capable of quantifying tibiofemoral kinematics dynamically in a cadaveric setting and with what accuracy?

Q5. How does this method compare to a skin marker system?

Q6. Is it feasible to quantify tibiofemoral kinematics in living subjects performing daily activities? 


\subsection{Aim and outline of thesis}

The main goal of this thesis is to describe the development and scientific assessment of the multi-channel A-mode ultrasound based motion capture method to effectively capture skeletal motion and to accurately quantify corresponding joint kinematics for the knee joint under dynamic conditions. Furthermore, we aimed at assessing the technical and clinical feasibility of this new ultrasound-based skeletal motion tracking system in both in-vitro and in-vivo experiments, and to demonstrate its potential for as an alternative method to measure human skeletal motion. The thesis consists of 6 chapters describing the following research topics:

Chapter 2 presents a numeric simulation followed by a cadaveric experiment to investigate the feasibility of utilizing a point cloud to register a known bone model. Furthermore, we assessed the effects of the number of used bony points and the magnitude of localization error of a bony point on the overall registration accuracy.

Chapter 3 demonstrates the technical feasibility of measuring tibiofemoral kinematics at different static positions in a human cadaveric experiment using a one-channel 3D-tracked A-mode ultrasound system. The work of this chapter guided the following development step towards a multi-channel A-mode ultrasound tracking system.

Chapter 4 presents a novel method to measure tibiofemoral kinematics dynamically using a multi-channel 3D-tracked A-mode ultrasound system. In this study we demonstrated the feasibility of this method and quantified its achievable accuracy in a cadaveric setting.

Chapter 5 presents in situ comparison between the multi-channel 3D-tracked A-mode ultrasound system and a conventional skin-mounted marker measurement on the achieved accuracies of estimated tibiofemoral kinematics in a cadaveric experiment. The purpose was to assess whether the newly develop system could overcome the effects of STA and mitigating its propagation to bone kinematics.

Chapter 6 presents an in-vivo demonstration study on the feasibility of measuring 6-DOF tibiofemoral kinematics in a group of healthy subjects. Various activities were considered while quantifying knee kinematics as well as the changes in soft tissue thickness. Hence, this chapter was meant to demonstrate a prototyping system that has great potential to measure human kinematics in an ambulant, non-radiative and non-invasive manner.

Chapter 7 presents a general discussion, where the key findings of this thesis are summarized, and the impact of the ultrasound-based skeletal motion tracking system on clinical applications is also discussed. In addition, future work is suggested for further improvements. 


\section{Introduction}

Finally, Chapter $\mathbf{8}$ was added as supplementary technical details for the development of the whole system which contained many technical challenges and which could not reasonably be described in the scientific publications (Chapters 2-6). Hence, this supplemental chapter provides valuable (technical and experimental) information regarding the different design stages of the system and also includes detailed descriptions on hardware characteristics and software design.

\section{References}

1. Ramsey, D.K. and P.F. Wretenberg, Biomechanics of the knee: methodological considerations in the in vivo kinematic analysis of the tibiofemoral and patellofemoral joint. Clinical Biomechanics, 1999. 14(9): p. 595-611.

2. Anderson, K.C., K.C. Buehler, and D.C. Markel, Computer assisted navigation in total knee arthroplasty: comparison with conventional methods. J Arthroplasty, 2005. 20(7 Suppl 3): p. 132-8.

3. Mavrogenis, A.F., et al., Computer-assisted navigation in orthopedic surgery. Orthopedics, 2013. 36(8): p. 631-42.

4. Sugano, N., et al., Accuracy evaluation of surface-based registration methods in a computer navigation system for hip surgery performed through a posterolateral approach. Comput Aided Surg, 2001. 6(4): p. 195-203.

5. Richard, V., A. Cappozzo, and R. Dumas, Comparative assessment of knee joint models used in multi-body kinematics optimisation for soft tissue artefact compensation. $\mathrm{J}$ Biomech, 2017.

6. Bingham, J. and G. Li, An Optimized Image Matching Method for Determining InVivo TKA Kinematics with a Dual-Orthogonal Fluoroscopic Imaging System. Journal of Biomechanical Engineering, 2006. 128(4): p. 588-595.

7. Dennis, D.A., et al., In vivo determination of normal and anterior cruciate ligamentdeficient knee kinematics. Journal of Biomechanics, 2005. 38(2): p. 241-253.

8. Gray, H.A., S. Guan, and M.G. Pandy, Accuracy of mobile biplane X-ray imaging in measuring 6-degree-of-freedom patellofemoral kinematics during overground gait. Journal of Biomechanics, 2017. 57: p. 152-156.

9. Bae, D.K. and S.J. Song, Computer Assisted Navigation in Knee Arthroplasty. Clinics in Orthopedic Surgery, 2011. 3(4): p. 259-267. 
10. Lafortune, M.A., et al., Three-dimensional kinematics of the human knee during walking. J Biomech, 1992. 25(4): p. 347-57.

11. Benoit, D.L., et al., Effect of skin movement artifact on knee kinematics during gait and cutting motions measured in vivo. Gait \& Posture, 2006. 24(2): p. 152-164.

12. Forsberg, D., et al., Quantitative analysis of the patellofemoral motion pattern using semi-automatic processing of 4D CT data. Int J Comput Assist Radiol Surg, 2016. 11(9): p. 1731-41.

13. Barratt, D.C., et al., Self-calibrating 3D-ultrasound-based bone registration for minimally invasive orthopedic surgery. IEEE Trans Med Imaging, 2006. 25(3): p. 312-23.

14. Mahfouz, M.R., et al., A robust method for registration of three-dimensional knee implant models to two-dimensional fluoroscopy images. IEEE Transactions on Medical Imaging, 2003. 22(12): p. 1561-1574.

15. Kaiser, J., et al., Measurement of 3D Tibiofemoral Kinematics using Volumetric SPGR-VIPR Imaging. Magnetic resonance in medicine : official journal of the Society of Magnetic Resonance in Medicine / Society of Magnetic Resonance in Medicine, 2013. 69(5): p. 1310-1316.

16. Reinschmidt, C., et al., Tibiofemoral and tibiocalcaneal motion during walking: external vs. skeletal markers. Gait \& Posture, 1997. 6(2): p. 98-109.

17. Akbarshahi, M., et al., Non-invasive assessment of soft-tissue artifact and its effect on knee joint kinematics during functional activity. Journal of Biomechanics, 2010. 43(7): p. 1292-1301.

18. Cappozzo, A., et al., Surface-marker cluster design criteria for 3-D bone movement reconstruction. IEEE Transactions on Biomedical Engineering, 1997. 44(12): p. 1165-1174.

19. Andersen, M.S., M. Damsgaard, and J. Rasmussen, Kinematic analysis of overdeterminate biomechanical systems. Comput Methods Biomech Biomed Engin, 2009. 12(4): p. 371-84.

20. Bonnechère, B., et al., Physiologically corrected coupled motion during gait analysis using a model-based approach. Gait \& Posture, 2015. 41(1): p. 319-322.

21. Charlton, I.W., et al., Repeatability of an optimised lower body model. Gait \& Posture, 2004. 20(2): p. 213-221.

22. Duprey, S., L. Cheze, and R. Dumas, Influence of joint constraints on lower limb kinematics estimation from skin markers using global optimization. Journal of Biomechanics, 2010. 43(14): p. 2858-2862.

23. Lu, T.W. and J.J. O'Connor, Bone position estimation from skin marker coordinates using global optimisation with joint constraints. Journal of Biomechanics, 1999. 32(2): p. 129-134. 
24. Andersen, M.S., et al., Do kinematic models reduce the effects of soft tissue artefacts in skin marker-based motion analysis? An in vivo study of knee kinematics. Journal of Biomechanics, 2010. 43(2): p. 268-273.

25. Cereatti, A., U. Della Croce, and A. Cappozzo, Reconstruction of skeletal movement using skin markers: comparative assessment of bone pose estimators. Journal of NeuroEngineering and Rehabilitation, 2006. 3: p. 7-7.

26. Baka, N., et al., Evaluation of automated statistical shape model based knee kinematics from biplane fluoroscopy. Journal of biomechanics, 2014. 47(1): p. 122-129.

27. Anderst, W., et al., Validation of Three-Dimensional Model-Based Tibio-Femoral Tracking During Running. Medical engineering \& physics, 2009. 31(1): p. 10-16.

28. Giphart, J.E., et al., Accuracy of a contour-based biplane fluoroscopy technique for tracking knee joint kinematics of different speeds. Journal of Biomechanics, 2012. 45(16): p. 2935-2938.

29. Banks, S. and P. Flood, JOINTTRACK AUTO: AN OPEN-SOURCE PROGRAMME FOR AUTOMATIC MEASUREMENT OF 3D IMPLANT KINEMATICS FROM SINGLE- OR BI-PLANE RADIOGRAPHIC IMAGES. Bone \&amp; Joint Journal Orthopaedic Proceedings Supplement, 2016. 98-B(SUPP 1): p. 38-38.

30. Li, G., S.K. Van de Velde, and J.T. Bingham, Validation of a non-invasive fluoroscopic imaging technique for the measurement of dynamic knee joint motion. $\mathrm{J}$ Biomech, 2008. 41(7): p. 1616-22.

31. Guan, S., et al., Mobile Biplane X-Ray Imaging System for Measuring 3D Dynamic Joint Motion During Overground Gait. IEEE Trans Med Imaging, 2016. 35(1): p. 326-36.

32. Kozanek, M., et al., Tibiofemoral kinematics and condylar motion during the stance phase of gait. Journal of Biomechanics, 2009. 42(12): p. 1877-1884.

33. List, R., et al., A moving fluoroscope to capture tibiofemoral kinematics during complete cycles of free level and downhill walking as well as stair descent. PLoS One, 2017. 12(10): p. e0185952.

34. Soavi, R., et al., A roentgen stereophotogrammetric analysis of unicompartmental knee arthroplasty. The Journal of Arthroplasty, 2002. 17(5): p. 556-561.

35. Garling, E.H., et al., Marker Configuration Model-Based Roentgen Fluoroscopic Analysis. Journal of Biomechanics, 2005. 38(4): p. 893-901.

36. Li, G., S.K. Van de Velde, and J.T. Bingham, Validation of a non-invasive fluoroscopic imaging technique for the measurement of dynamic knee joint motion. Journal of Biomechanics, 2008. 41(7): p. 1616-1622.

37. Bontempi, M., et al., Total knee replacement: intraoperative and postoperative kinematic assessment. Acta Biomed, 2017. 88(2 -s): p. 32-37. 
38. Mazzoli, V., et al., Accelerated 4D self-gated MRI of tibiofemoral kinematics. NMR Biomed, 2017.

39. Clarke, E.C., et al., A non-invasive, 3D, dynamic MRI method for measuring muscle moment arms in vivo: Demonstration in the human ankle joint and Achilles tendon. Medical Engineering \& Physics, 2015. 37(1): p. 93-99.

40. Zhao, K., et al., A Technique for Quantifying Wrist Motion Using FourDimensional Computed Tomography: Approach and Validation. Journal of Biomechanical Engineering, 2015. 137(7): p. 0745011-0745015.

41. Chan, V. and A. Perlas, Basics of Ultrasound Imaging. 2011: p. 13-19.

42. Dehghan, E., et al., Ultrasound-fluoroscopy registration for prostate brachytherapy dosimetry. Med Image Anal, 2012. 16(7): p. 1347-58.

43. Banerjee, J., et al., Fast and robust 3D ultrasound registration - Block and game theoretic matching. Medical Image Analysis, 2015. 20(1): p. 173-183.

44. Marotti, J., et al., Recent advances of ultrasound imaging in dentistry--a review of the literature. Oral Surg Oral Med Oral Pathol Oral Radiol, 2013. 115(6): p. 819-32.

45. Amin, D.V., et al., Ultrasound registration of the bone surface for surgical navigation. Comput Aided Surg, 2003. 8(1): p. 1-16.

46. Chen, T., et al., A system for ultrasound-guided computer-assisted orthopaedic surgery. Computer Aided Surgery, 2005. 10(5-6): p. 281-292.

47. Talib, H., et al., Information filtering for ultrasound-based real-time registration. IEEE Trans Biomed Eng, 2011. 58(3): p. 531-40.

48. Murtha, P.E., et al., Accuracy of ultrasound to MR registration of the knee. The International Journal of Medical Robotics and Computer Assisted Surgery, 2008. 4(1): p. 5157.

49. Chang, T.C., et al., A-Mode Ultrasound Bone Registration for Computer-Assisted Knee Surgery: Calibration and Robustness Test, in 25th Southern Biomedical Engineering Conference 2009, 15 - 17 May 2009, Miami, Florida, USA, A. McGoron, C.-Z. Li, and W.C. Lin, Editors. 2009, Springer Berlin Heidelberg. p. 97-100.

50. Fieten, L., et al., Fast and accurate registration of cranial CT images with A-mode ultrasound. Int J Comput Assist Radiol Surg, 2009. 4(3): p. 225-37.

51. Mozes, A., et al., Three-dimensional A-mode ultrasound calibration and registration for robotic orthopaedic knee surgery. Int J Med Robot, 2010. 6(1): p. 91-101.

52. Popovic, A., et al., Efficient non-invasive registration with A-mode ultrasound in skull surgery. CARS 2005: Computer Assisted Radiology and Surgery, 2005. 1281: p. 821826.

53. Amstutz, C., et al., A-mode ultrasound-based registration in computer-aided surgery of the skull. Arch Otolaryngol Head Neck Surg, 2003. 129(12): p. 1310-6. 


\section{Introduction}

54. Oszwald, M., et al., Accuracy of navigated surgery of the pelvis after surface matching with an a-mode ultrasound probe. J Orthop Res, 2008. 26(6): p. 860-4.

55. Masum, M.A., et al., Accuracy assessment of Tri-plane B-mode ultrasound for noninvasive 3D kinematic analysis of knee joints. Biomed Eng Online, 2014. 13: p. 122.

56. Jia, R., et al., CAT \& MAUS: A novel system for true dynamic motion measurement of underlying bony structures with compensation for soft tissue movement. Journal of Biomechanics, 2017.

57. De Lorenzo, D., et al. Experimental validation of A-mode ultrasound acquisition system for computer assisted orthopaedic surgery. in Medical Imaging 2009: Ultrasonic Imaging and Signal Processing. 2009. Lake Buena Vista, FL.

58. Hamidzada, W.A. and E.P. Osuobeni, Agreement between A-mode and B-mode ultrasonography in the measurement of ocular distances. Vet Radiol Ultrasound, 1999. 40(5): p. $502-7$.

59. Maurer, C., Jr., et al., AcouStick: A Tracked A-Mode Ultrasonography System for Registration in Image-Guided Surgery, in Medical Image Computing and Computer-Assisted Intervention - MICCAI'99, C. Taylor and A. Colchester, Editors. 1999, Springer Berlin Heidelberg. p. 953-962.

60. Grood, E.S. and W.J. Suntay, A joint coordinate system for the clinical description of three-dimensional motions: application to the knee. J Biomech Eng, 1983. 105(2): p. 13644. 
Feasibility of A-mode ultrasound based intraoperative registration in computer-aided orthopedic surgery: a simulation and experimental study

\section{Chapter 2 Feasibility of A-mode ultrasound based intraoperative registration in computer- aided orthopedic surgery: a simulation and experimental study}

Purpose: Computer-Aided Orthopedic Surgery (CAOS) requires a quick and accurate registration of patient's anatomy during operation to the pre-operatively acquired image data. In orthopedic surgery, single element or A-mode ultrasound (US) may provide fast and accurate registration without the requirement of a surgical incision. To utilize A-mode US for intraoperative registration in CAOS, a suitable registration algorithm is necessary with a small number of registration points and taking into account measurement errors (such as noise) of the ultrasound sensor. Therefore we investigated the effects of (1) the number of registration points and (2) the Ultrasound Point Localization Error (UPLE) on the overall registration accuracy. The final simulation outcomes were validated in a cadaver experiment and the feasibility of A-mode US based intraoperative registration in CAOS was assessed.

Methods: We developed a new registration method, including the Iterative Closest Points (ICP) algorithm and a Perturbation Search algorithm (ICP-PS). This method enables to avoid getting stuck in the local minimum of ICP iterations and to find the adjacent global minimum. This registration method was subsequently tested in a numerical simulation and a cadaveric experiment using a 3D-tracked A-mode US system.

Results: The results showed that ICP-PS outperformed the standard ICP algorithm. The accuracy of the proposed registration method improved with the addition of ultrasound registration points, but the rate of improvement gradually declined. In simulation, using 25 ultrasound registration points, an average registration accuracy of $0.25 \mathrm{~mm}$ was reached with zero UPLE. The accuracy error increased to $1.10 \mathrm{~mm}$ for $1 \mathrm{~mm}$ UPLE, and to $1.97 \mathrm{~mm}$ for $2 \mathrm{~mm}$ UPLE. In the cadaver experiment, using 25 registration points, an accuracy of $2.81 \mathrm{~mm}$ was reached.

Conclusions: The simulation approach provided a well-defined framework for estimating the necessary number of ultrasound registration points and acceptable level of UPLE for a given required level of accuracy for intraoperative registration in clinical practice. Our proposed registration method outperformed standard ICP in both numerical simulation and cadaveric experiment, which proved that ICP-PS is suitable for A-mode US based intraoperative registration. This study would facilitate the application of A-mode US probe in registering the point cloud to a known shape model (i.e. intraoperative registration for CAOS), which 
Feasibility of A-mode ultrasound based intraoperative registration in computer-aided orthopedic surgery: a simulation and experimental study

also has the potential of being used for accurately estimating bone position and orientation for skeletal motion tracking and surgical navigation. 
Feasibility of A-mode ultrasound based intraoperative registration in computer-aided orthopedic surgery: a simulation and experimental study

\subsection{Introduction}

Computer-Aided Orthopedic Surgery (CAOS) systems have been developed, validated and used for surgery in the lower extremity, such as total knee arthroplasty (TKA) [1,2] and total hip arthroplasty (THA) [3]. CAOS systems offer several advantages over traditional surgery: improving guidance of the surgical instruments, reducing complication rates, minimizing trauma from instrument access and allowing preview and measurement of anatomical regions in a virtual environment [4-6]. In some of CAOS scenarios, medical images of a patient are acquired preoperatively, for example from Computed Tomography (CT) or Magnetic Resonance Imaging (MRI), and used to plan the surgical steps. During surgery, the image data then needs to be registered to the actual patient. To find the transformation that matches the preoperative image coordinate system to the intraoperative patient coordinate system in order to express one data set in the coordinate system of other data set and vice versa, the first step is to acquire intraoperative data (e.g. digitized points, lines, curves or surfaces) from the anatomy of the actual patient in the operating room. The second step is to use an appropriate registration algorithm to determine the transformation.

The acquisition of intraoperative data can be done by using various types of markers such as adhesive skin markers [7,8], anatomical landmarks and implantable bone markers, all of which have advantages and disadvantages. Skin markers are non-invasive, but the skin movements relative to the bone may dramatically decrease the accuracy of registration [8]. Anatomical landmarks on specific locations of patient's anatomy can be detected and digitized utilizing pointer probes (tracked by an optical or electromagnetic navigation system) [9], but using anatomical landmarks often requires surgical exposure of additional bony surfaces, causing additional trauma and extension of the operating procedure. Implanted bone markers provide the highest registration accuracy, and are commonly considered as the gold standard for evaluating a registration algorithm for clinical application [10]. The implanted markers can be tracked using intraoperative fluoroscopy or CT data [11]. In cadaver experiments, the error has been reported to be $0.99 \pm 0.41 \mathrm{~mm}$ [12]. However, implanted markers have the drawback of exposing the patient to additional radiation and trauma. Because it is necessary to affix the implant markers to the patient before pre-operative images are acquired, which is an invasive and costly process that may require extra trauma and additional blood loss instead of primary surgical site. To avoid most of aforementioned drawbacks, ultrasound (US) offers a truly noninvasive and non-radiative way for intraoperative registration. As ultrasound is capable of visualizing the bone boundaries through the soft tissue, ultrasound-based intraoperative registration eliminates the need for physical contact with the bone surface [13]. 
Feasibility of A-mode ultrasound based intraoperative registration in computer-aided orthopedic surgery: a simulation and experimental study

A-mode (Amplitude modulation, a display of amplitude of received echo as a function of depth through a single transducer scanning) ultrasound has already been used for intraoperative registration [14-17]. Compared to the conventional B-mode (Brightness-mode, a display of 2D image that image intensity depends on the amplitude of received B-mode echoes through an array of transducers) ultrasound transducers, A-mode ultrasound transducers are cheaper and more suited for determining the bone surface in real-time [18, 19], when taking cost and workload of employing multiple ultrasound transducers into account. Besides, the size of A-mode ultrasound is beneficial to be installed in various configurations to fit different anatomical areas. Although A-mode ultrasound could not provide the $2 \mathrm{D}$ image like $\mathrm{B}$-mode ultrasound, the received ultrasound signal is enough for depth detection. Its localization accuracy was reported to be approximately $0.4 \mathrm{~mm}$ after calibration $[14,16,20]$. However, since the calibration procedure is indispensable, it would need an additional procedure before intraoperative registration. The combination of a CAOSsystem and single A-mode ultrasound transducer has been used in skull surgery [21, 22], pelvis surgery [23], and knee surgery [16].

Hence, to utilize A-mode ultrasound transducers for intraoperative registration with its inherent localization error, a clearly defined registration procedure should be established. Different from the image registration by using image intensity or other image related information, A-mode based registration typically uses a $3 \mathrm{D}$ point cloud obtained by the transducer to register on a known shape model of the bony segment. For the registration, the Iterative Closest Points (ICP) algorithm is commonly used to compute the transformation between the point cloud and shape model by minimizing the objective function iteratively that usually is a distance function from the point cloud to the corresponding points on the shape model, such as the sum of squared differences of Euclidean distances between the matched point pairs [24]. Generally, a larger number of points will probably lead to a more accurate registration, but herein a tradeoff lies between registration accuracy and the time spent on point acquisition. In this study, the combination of ICP with a Perturbation Search algorithm was described as a suitable method for A-mode ultrasound based intraoperative registration. This method attempts to avoid getting stuck in the local minimum of ICP iterations and to complete accurate registration with less registration points than conventional ICP would require $[16,25]$. The registration method was subsequently tested by means of a numerical simulation and a cadaveric experiment.

Furthermore, it is likely that the registration accuracy will not only depend on the number of points acquired, but also on the errors to accurately locate the true points (termed Ultrasound Point Localization Error, UPLE). To assess the sensitivity of these aspects in a systematic manner, in-vivo or in-vitro studies would not be ideal as the first step as it is very 
Feasibility of A-mode ultrasound based intraoperative registration in computer-aided orthopedic surgery: a simulation and experimental study

difficult to change one factor while keeping the others constant. We therefore first performed a numerical simulation (i.e. Monte-Carlo simulation) to determine the effects of two aspects on the accuracy of both our proposed registration method and the standard ICP: (1) the number of ultrasound registration points and (2) the point localization errors (UPLE) introduced by A-mode ultrasound point detection. Secondly, a cadaveric experiment was conducted to verify the outcomes of the simulation and to test the proposed registration method in practice.

\subsection{Materials and Methods}

\subsubsection{Data generation for simulation}

In this study, we focus on the femur bone as object of study. A shape model (a STL file format, contains 37745 vertices) of a healthy femoral bone was generated from CT data in a previous study[26]. This model was considered as a known shape model that represents the preoperative data of the patient (termed the Pre-Operative Model, POM), which will also be used for generating the ultrasound registration points.

In the clinical registration procedure, the points measured by A-mode ultrasound on the bony surface of the actual patient (termed the Surface Sample Points, SSP) can thus be registered to the POM that was segmented on the preoperative CT/MRI images, as shown in Figure 2.1.

The simulated registration procedure starts with the acquisition of a set of points from the POM. A set of points is selected from all areas that would be accessible to ultrasound without incision[16] as shown in Figure 2.2. These selected points were considered as the ground truth locations of the SSP after registration (termed GT-SSP). After selecting GT-SSP, to generate the corresponding SSP in the patient coordinate system, the GT-SSP was transformed to an arbitrary new location, resulting in a set of ultrasound sample points (i.e. SSP) on a virtual patient coordinate system. The selection of GT-SSP was performed by the following two-step protocol:

(1) The first six points of the GT-SSP were picked from three pre-defined restricted areas, termed pre-registration areas. These areas are clinically easy to locate and detect. To simulate different conditions that would happen in the practical situation, two points were randomly selected from each pre-registration area (Figure 2.2): two points from the greater trochanter area, two points from the medial epicondyle area and two points from the lateral epicondyle area. These six points were intended for pre-registration of the SSP to the POM, which will be discussed later. 
Feasibility of A-mode ultrasound based intraoperative registration in computer-aided orthopedic surgery: a simulation and experimental study

(2) The rest of the GT-SSP were considered as the additional points for registration and they were randomly picked from all accessible areas (as shown in Figure 2.2).

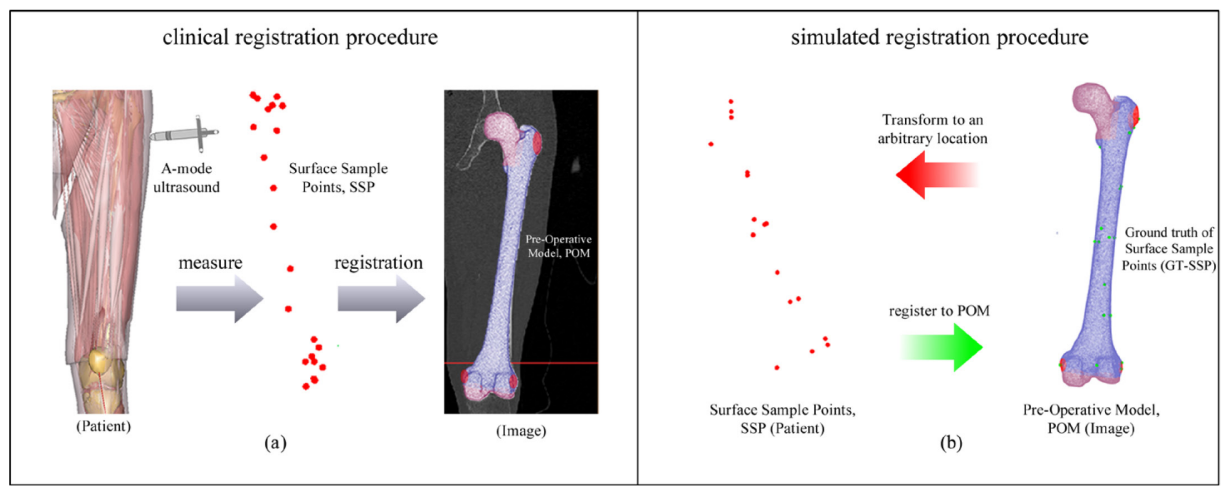

Figure 2.1 The comparison between clinical registration procedure and simulation registration. (a) the procedure of clinical registrattion procedure by measuring ultrasound points from patient and registering to the pre-operative model. (b) the procedure of simulated registration through generating the ultrasound point from pre-operative model and transforming to an new position rigidly, eventually registering those points back to the pre-operative model.

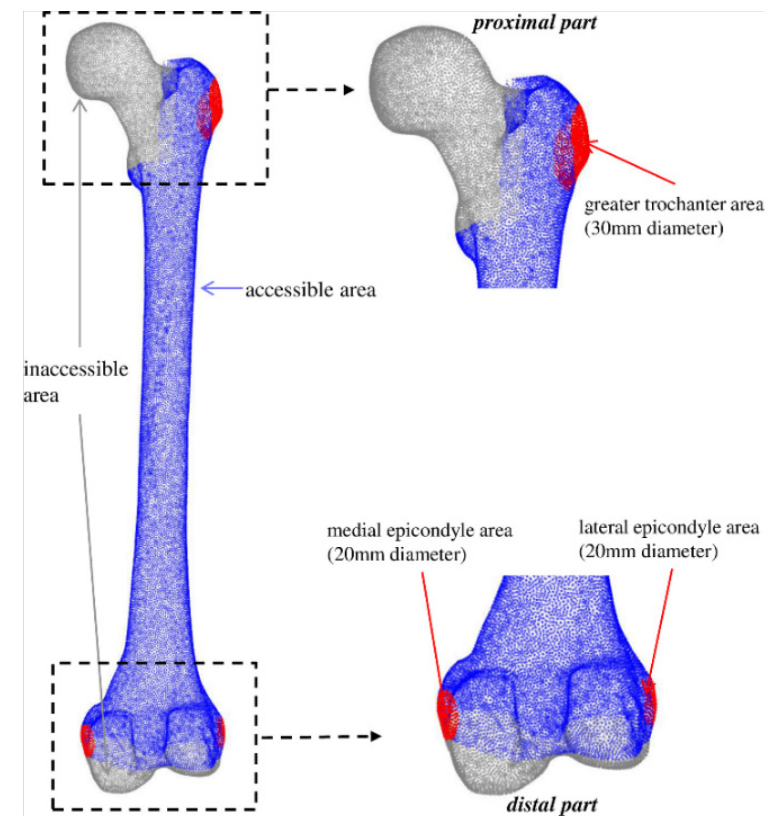

Figure 2.2 The inaccessible areas (gray), accessible areas (blue) and pre-registration areas (red) on Preoperative Model. The pre-registration areas include: the greater trochanter area $(30 \mathrm{~mm}$ diameter), medial epicondyle area $(20 \mathrm{~mm}$ diameter) and lateral epicondyle area $(20 \mathrm{~mm}$ diameter); shaft areas around the middle shaft of the femur: lateral, medial, anterior, posterior (20 diameter for each side). 
Feasibility of A-mode ultrasound based intraoperative registration in computer-aided orthopedic surgery: a simulation and experimental study

\subsection{Registration method}

The goal is now to register the SSP to the POM and to calculate the transformation between the patient coordinate system and the image coordinate system (i.e. to link the patient in the operating room to the preoperatively scanned image data). The registration is performed in a four-step procedure: First, a point-to-point preregistration is performed using the 6 points from the pre-registration areas. Secondly an Iterative Closest Point (ICP) algorithm is applied using all of the points from the SSP to register to the POM. Thirdly, to avoid local minima in the ICP, a systematic perturbation is applied exploring if there are better registration results available compared to the first ICP result, based on the Point-to-Surface Euclidean Distance (PSD) between the registered SSP and the POM. Fourth, the feedback loop that includes perturbation and followed by the ICP is then repeated until the convergence of the PSD is reached. Each of these steps is described in more detail in the following sections.

\subsubsection{Pre-registration: $1^{\text {st }}$ step}

The first step is a pre-registration (coarse registration) using only the six points from the greater trochanter and both lateral and medial femoral epicondyles. This is achieved using point-to-point registration that fits the first six points of the SSP to a set of corresponding points that represent the centroids of the pre-registration areas. Point-to-Point rigid registration is a process that finds the transformation for the minimal distance between target points and measured points[27]. The objective function is defined as:

$$
f(\boldsymbol{R}, \boldsymbol{T})=\frac{1}{n} \sum_{i=1}^{n}\left\|x_{i}-\left(\boldsymbol{R} u_{i}+\boldsymbol{T}\right)\right\|^{2}
$$

where $U=\left\{u_{i}\right\}$ is a set of first $n$ points of SSP ( $n=6$ in our simulation) and $X=\left\{x_{i}\right\}$ is the set of all the corresponding points on POM, which are centroids of pre-registration areas, $\boldsymbol{T}$ represents the translation vector and $\boldsymbol{R}$ represents the rotation matrix between the SSP and the POM.

\subsubsection{Iterative Closest Point (ICP): $2^{\text {nd }}$ step}

The maximum number of iterations of the ICP algorithm was set to 30 in our simulation and experiment. The details of ICP algorithm can be found in [24]. For each iteration, firstly, the closest points on the POM with respect to the SSP were calculated. Secondly, a point-topoint registration was applied on the corresponding point pairs between SSP and closest points to get a updated SSP. Then the iterative procedure repeats first and second steps until meeting ending conditions (beyond the iteration times or convergence). To speed up the ICP 
Feasibility of A-mode ultrasound based intraoperative registration in computer-aided orthopedic surgery: a simulation and experimental study

algorithm, k-d tree [28] was used for searching the closest points of the SSP from the POM, which is the most time consuming part in the ICP algorithm.

\subsubsection{Perturbation Search: $3^{\text {rd }}$ step}

As ICP and its variants are local optimization methods, which can get stuck in local minima of the objective functions, it is difficult to find the global minimum from an arbitrary starting position without the pre-registration process [29]. To avoid local minima of the ICP registration, our method was inspired by the method of Ma and Ellis[29]. We perturbed the transformed SSP rigidly from its ICP registered position and verified whether the perturbed solution represented a smaller local minimum or even a global one. Perturbation can obviously be done in all directions. Instead of perturbing randomly, we, based on a pilot study, assumed that most mismatching occurred along the distal-proximal axis of the femur rather than the anterior-posterior axis or the lateral-medial axis (Figure 2.3). The reason for this also lies in the fact that the femur has a somewhat cylinder-like shape. Especially when the number of ultrasound registration points is too small for providing strong geometrical constraints in all directions (missing points from the proximal and distal parts of the femur), it is more likely for translational and rotational misalignments to occur around the distalproximal axis of the femur. The perturbation of the first ICP result was therefore implemented as a rotation around the femoral-distal-proximal axis from -5 to 5 degrees with intervals of 1 degree and a translation along the same axis from -3 to $3 \mathrm{~mm}$ with intervals of $0.5 \mathrm{~mm}$. The perturbation can thus be seen as a curved grid around the bone of 143 (11 by 13 grid) combinations of rotations and translations (Figure 2.3). For each perturbation along this curved grid, the Point-to-Surface Euclidean Distance (PSD) was calculated and compared to the PSD of the original ICP result:

$$
\mathrm{PSD}=\frac{1}{n} \sum_{i=1}^{n}\left\|s_{i}-u_{i}\right\|^{2}
$$

where $S=\left\{s_{i}\right\}$ is a set of $n$ closest points on the Preoperative Model's surface. Each $s_{i}$ is calculated by each $u_{i}$, and $U=\left\{u_{i}\right\}$ is perturbed sample points after ICP. 
Feasibility of A-mode ultrasound based intraoperative registration in computer-aided orthopedic surgery: a simulation and experimental study

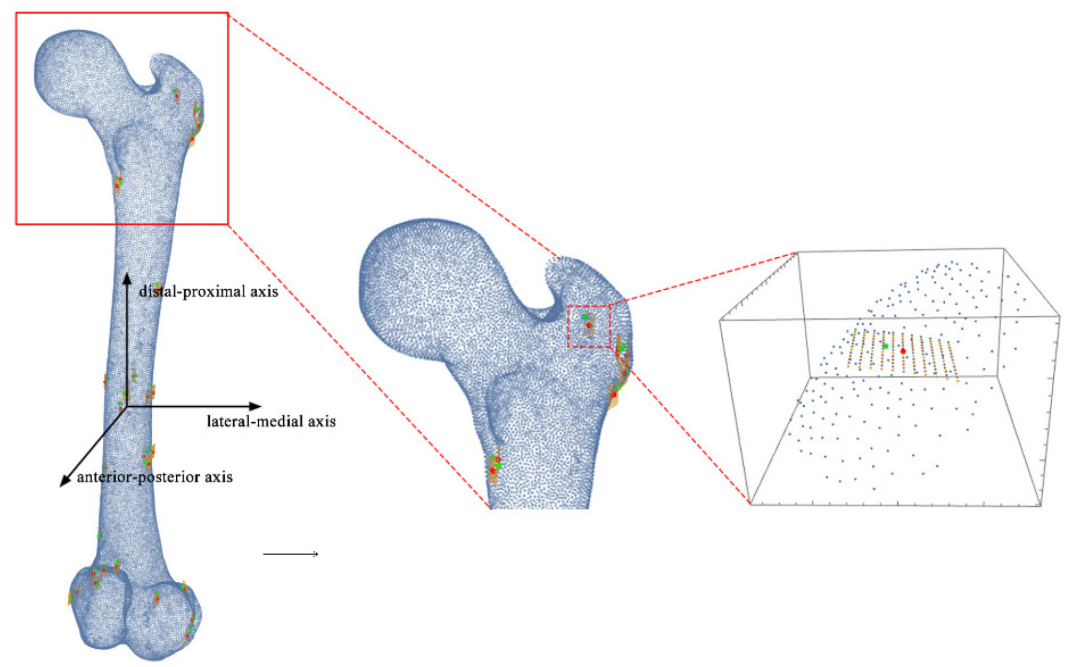

Figure 2.3 The illustration of Perturbation Search. The curved grid of perturbation searches with 143 combinations of rotations and translations for each point in simulation environment. Green point represents the perturbed sample points after ICP. Red point represent the registered points of ICP.

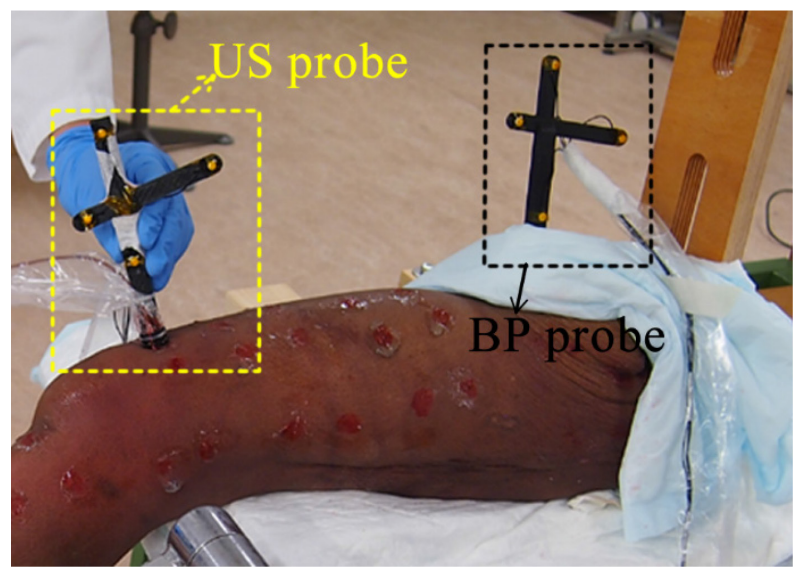

Figure 2.4 The setup of cadaver experiment and ultrasound probe and bone pin probe. The ultrasound probe and bone pin probe have four optical markers. The bone pin probe provides the ground truth position of the underlying bone.

\subsubsection{Feedback from perturbation to ICP: $4^{\text {th }}$ step}

If the perturbation procedure produces a more optimal result than the ICP result from the second step (section 2.2.2), the ICP algorithm is repeated from the position provided by the perturbation. The feedback loop that includes perturbation and followed by the ICP is then 
Feasibility of A-mode ultrasound based intraoperative registration in computer-aided orthopedic surgery: a simulation and experimental study

repeated until the convergence of the PSD is reached, i.e. $\mathrm{PSD}_{\mathrm{n}+1}-\mathrm{PSD}_{\mathrm{n}}<0.00001$, with a maximum of 5 repetitions.

\subsubsection{Definition of registration accuracy}

The accuracy of the registration was estimated by calculating the root mean square error (RMSE) of Euclidean distances between the registered POM (i.e. the POM corresponding to the registered SSP), $U=\left\{u_{i}\right\}$ and the known ground truth of the POM (i.e. corresponding to the GT-SSP), $G=\left\{g_{i}\right\}$, where $n$ represents the number of points in the POM. Therefore, the RMSE calculation represents a bone -to- bone (point-to-point for all corresponding point pairs, $i=37745$ ) error metric:

$$
\text { RMSE }=\sqrt{\frac{1}{n} \sum_{i=1}^{n}\left(\left\|u_{i}-g_{i}\right\|^{2}\right)^{2}}
$$

\subsection{Simulation procedure}

In the simulation, the aim of this study was to assess the accuracy of our proposed registration method as a function of the number of registration points and UPLE. To compare our proposed method to the standard ICP, the accuracy of different steps of our registration method was calculated after the second, third and fourth step of the registration procedure as described in the previous section $(2.2 .1-2.2 .4)$. The number of registration points ranged from 6 to 25 (i.e. 20 different sizes for registration points). The UPLE (i.e. the noise of SSP) has several sources, e.g. calibration error (caused by calibration procedure approximate 0.4 $\mathrm{mm})$ [16], speed of sound that would affect the depth calculation $(v t / 2$; where $v$ is the velocity of sound in the material $(1590 \mathrm{~m} / \mathrm{s}$ in muscle across the fibers [30] and $t$ represents the time that ultrasound waveform takes from the origin of ultrasound beam to the bone surface and reflect back to the origin.) [31], navigation system (intrinsic error of navigation system, depending on deployed system). An investigation of the noise model of SSP is beyond the scope of this study, hence, we assumed the noise had isotropic distribution in all direction and was added to SSP randomly. The UPLE was simulated as a noise vector in random direction with the magnitude uniformly distributed over a preset interval[32]. The interval was varied from [0] (i.e. zero UPLE), [0-1] and [1-2] $\mathrm{mm}$. We chose this range based on previous studies in ultrasound detection that reported errors varied between 0.5 and $2 \mathrm{~mm}$ after calibration $[16,20,22]$. Then, to mitigate the effect of the randomness in the distribution of the SSP (i.e. the points selected in step two of the protocol as described in section 2.1), at the same range of UPLE and for each size of the SSP, the procedure from selecting GT-SSP 
Feasibility of A-mode ultrasound based intraoperative registration in computer-aided orthopedic surgery: a simulation and experimental study

to applying registration were repeated a hundred times. In total, we thus ran $6000(20 \mathrm{x} 3 \mathrm{x}$ 100) simulations.

The simulation program was implemented in Mathematica (Wolfram Mathematica 10.3.0). The computer hardware configuration used was Intel Core i7-4800MQ $(2.70 \mathrm{GHz})$ and $8 \mathrm{G}$ RAM. The time efficiency of a single case of simulation (including from Select GT-SSP to whole registration procedure) was 11.08 seconds on average. The total time cost of all simulations combined was about 18 hours.

\subsection{Cadaver experiment}

In this study we used one human cadaveric specimen. Testing on cadaveric knees is necessary to ensure the safety and functioning of the developed technology before we apply this to patients. At the Radboud University Medical Center (Radboud UMC) we have a long history of performing cadaveric experiments and we established working principles to ensure all ethical issues and legal aspects are covered. Radboud UMC has an Anatomical department which has the authority under Dutch law to use human tissue for educational and research purposes. The Dean of the Medical faculty is responsible for the ethical issues with regard to the use of human cadaver material. The cadaveric experiment was conducted according to the protocol issued by the department of anatomy of the Radboud UMC and were approved by both our local ethics advisor and the European Research Council (UMCRPS02PRD_PRTC0119). None of the transplant donors were from a vulnerable population and all donors or next of kin provided written informed consent that was freely given.

To verify the outcomes of the simulation and assess the performance of our method in practice, a cadaver experiment was conducted on a left leg. Prior to CT scan, one Bone Pin probe (BP probe) was screwed onto the proximal part of femur, with a frame containing four optical markers (Figure 2.4). Then a CT scan was made at the department of Radiology of the Radboud UMC using TOSHIBA Aquilion ONE (TOSHIBA, Tustin, USA) with voxel size of $0.755 \mathrm{~mm} \times 0.755 \mathrm{~mm} \times 0.500 \mathrm{~mm}$. The image was segmented manually in Mimics ${ }^{\circledR}$ 17.0 (Materialise N.V., Leuven, Belgium) and the surface model of the femur was generated for registration. The 3D locations of four optical markers of the BP probe were also digitized manually from the CT data. During the experiment, the femoral head was fixated in a custom setup and the upper leg was kept stationary during experiment, as shown in Figure 2.4. One Ultrasound probe (US probe) that contained four optical markers and one 7.5 MHz A-mode ultrasound transducer (Imasonic SAS, Voray / l'Ognon, France) was used to acquire ultrasound sample points. An optical tracking system (Visualeyez VZ4000v trackers, PTI Phoenix Technologies Inc, Vancouver, Canada) was operated at $100 \mathrm{~Hz}$ to track the 3D 
Feasibility of A-mode ultrasound based intraoperative registration in computer-aided orthopedic surgery: a simulation and experimental study

locations of BP probe and US probe. The ultrasound signal was captured and synchronized with the optical tracking system in the Diagnostic Sonar FI Toolbox (Diagnostic Sonar Ltd., Livingston, Scotland). Custom written software was developed to process all data in LabVIEW 2014 (National Instruments, Austin, USA). The origin and direction of ultrasound beam were determined from the calibration method [16]. The ultrasound echo signal was filtered and a peak detection window was manually set to find the maximum peak and thus determine the bone depth. Subsequently, the ultrasound sample point could be computed [20].

The protocol to define the locations where points were collected was similar to the protocol in the simulated registration procedure (section 2.1). Instead of randomly acquiring points, we measured points from 25 anatomical areas including pre-registration areas on the upper leg, which were distributed homogeneously and covered anterior, lateral and medial parts of femur. For each anatomical area, the US probe acquisition was repeated 10 times. After acquiring all points, the UPLE of each point was estimated by calculating the PSD between each point and the ground truth location of bone determined from the BP probe. To assess the effect of UPLE in this in vitro case, both the maximal PSD case (i.e. sample point located farthest from bone) and the minimal PSD case (points closest to bone) out of 10 points from each anatomical area were selected. After data acquisition, registration was performed for 6 points increasing to 25 points in descending order of accuracy for both the maximal and minimal case. In the rest of this study, these two datasets will be referred to as "closest" and "farthest". Both standard ICP and ICP-PS method were applied onto these two cases. The registration accuracies of these two registration methods were estimated by computing the RMSE of distance between registered bone position and the gold standard position derived from the BP probe.

\subsection{Results}

\subsubsection{Simulation: effect of the registration step for different UPLE}

Figure 2.5 shows the average RMSE of the resulting registration at several steps in the registration procedure as a function of the number of sample points. With the UPLE set to zero, after the second step (ICP), the average RMSE ranged from $4.61 \mathrm{~mm}$ for 6 sample points to $1.71 \mathrm{~mm}$ for 25 sample points (Figure 2.5-a). After the third step (perturbation search), this dropped to an average RMSE of $4.51 \mathrm{~mm}$ for 6 points and to an average RMSE of $0.76 \mathrm{~mm}$ for 25 points (Figure 2.5-a). The fourth step, (i.e. repeating ICP and perturbation search until convergence is reached) yielded a further drop of the average RMSE, to $4.39 \mathrm{~mm}$ for 6 sample points and $0.25 \mathrm{~mm}$ for 25 sample points after the first iteration (Figure 2.5-a). 
Feasibility of A-mode ultrasound based intraoperative registration in computer-aided orthopedic surgery: a simulation and experimental study

The addition of UPLE to the sample points clearly reduced the accuracy of the registration. With the UPLE set to $[0,1]$, after the second step (ICP), the average RMSE was $4.66 \mathrm{~mm}$ for 6 sample points and $1.84 \mathrm{~mm}$ for 25 sample points. After the fourth step, the average RMSE was $4.62 \mathrm{~mm}$ for 6 sample points and $1.10 \mathrm{~mm}$ for 25 sample points. When the UPLE set to $[1,2]$, the average RMSE after the second step (ICP) was $4.57 \mathrm{~mm}$ for 6 sample points and $2.15 \mathrm{~mm}$ for 25 sample points. After fourth step, the average RMSE was $5.20 \mathrm{~mm}$ for 6 sample points and $1.97 \mathrm{~mm}$ for 25 sample points. The improvement of registration accuracy from second step to fourth step became less effective with increasing UPLE. The number of iterations needed to reach convergence within the fourth step never exceeded eight.

\subsubsection{Simulation: effect of the number of registration points for different UPLE}

Generally, the registration accuracies of both standard ICP and our method improved by increasing the number of sample points for all three UPLE cases. With a non-zero UPLE i.e. $[0,1]$ or $[1,2]$, the average RMSE still decreased with increasing the number of points, but the rate of descent declined with increasing UPLE (Figure 2.5).

With the UPLE set to zero, standard ICP achieved an average RMSE with $1.71 \mathrm{~mm}$ by using 25 sample points. Using ICP-PS, the average RMSE dropped to $1.27 \mathrm{~mm}$ at merely 13 points and reached a level of $0.25 \mathrm{~mm}$ at 25 points. With the UPLE set to [0,1], using 25 sample points could reach an average RMSE with $1.84 \mathrm{~mm}$. For our method, only 15 sample points were needed to reach even higher accuracy (average RMSE: $1.40 \mathrm{~mm}$ ), as shown in Figure 2.5-b. Nevertheless, this outperformance of our registration method that achieved higher accuracy with fewer sample points vanished when the UPLE set to $[1,2]$. The performance of our method was even worse than standard ICP when the number of points was lower than 16 and was only slightly better with more than 16 points (Figure 2.5 -c).

\subsubsection{Experimental results: cadaver experiment}

Figure 2.6 shows the resulting RMSE for the "farthest" and the "closest" cases with an increasing number of points and comparing standard ICP to ICP-PS (Figure 2.6). When the number of points was beyond 8 , our method provided a more accurate registration result than standard ICP for both cases. In the "farthest" case, when the number of points was 25 , the RMSE was $5.88 \mathrm{~mm}$ for ICP method and was $3.96 \mathrm{~mm}$ for ICP-PS. In the "closest" case, ICP-PS achieved an RMSE of $2.81 \mathrm{~mm}$ relative to $5.84 \mathrm{~mm}$ for the ICP for the identical 25 points. 
Feasibility of A-mode ultrasound based intraoperative registration in computer-aided orthopedic surgery: a simulation and experimental study
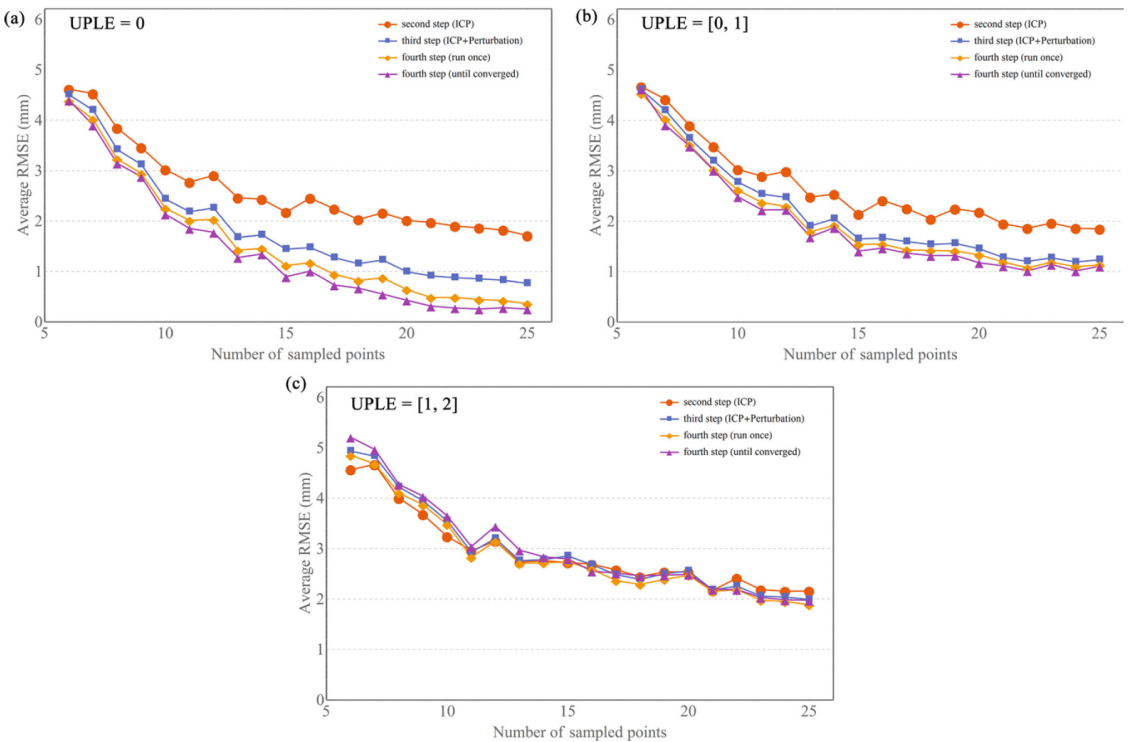

Figure 2.5 The simulation results of average RMSE after different registration steps. (a) the average RMSE after different registration steps with the UPLE set to zero. (b) the average RMSE after different registration steps with the UPLE set to $[0,1]$. (c) the average RMSE after different registration steps with the UPLE set to $[1,2]$.
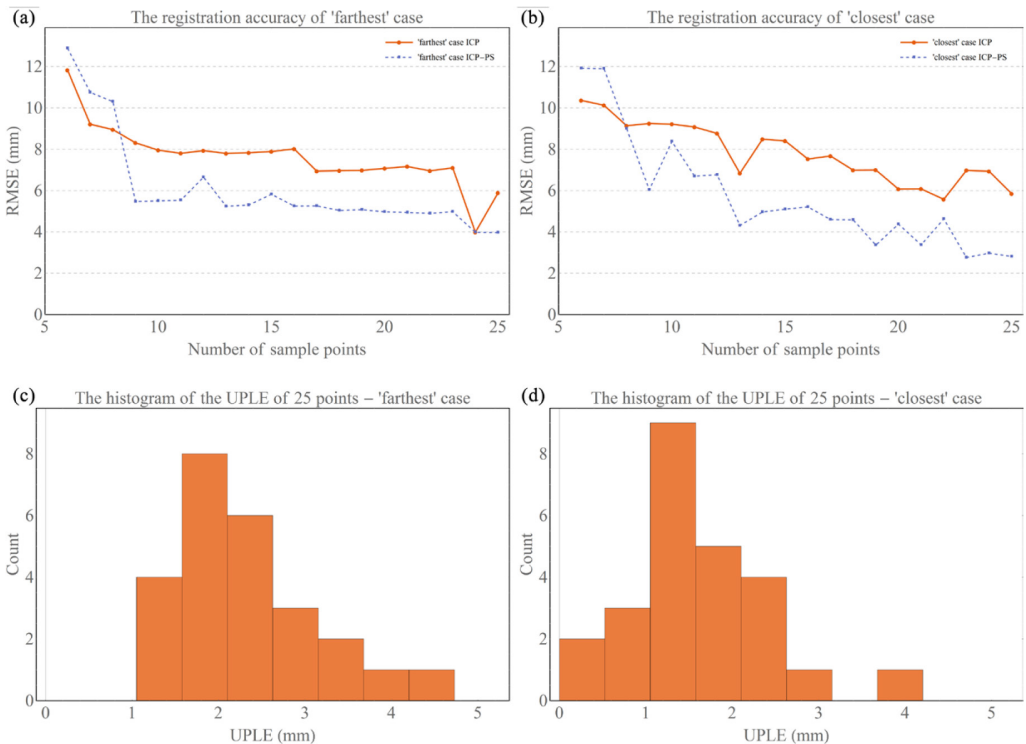

Figure 2.6. The cadaver experiment results of registration accuracy and UPLE. (a) The registration accuracy of different registration methods on the "farthest" case. (b) The registration accuracy of different registration methods on the "closest" case. (c) the histogram of the UPLE of 25 point for the "farthest" case. (d) the histogram of the UPLE of 25 points for the "closest" case. 
Feasibility of A-mode ultrasound based intraoperative registration in computer-aided orthopedic surgery: a simulation and experimental study

The histograms of the UPLE of 25 points for two cases is shown in Figure 2.6-c and Figure 2.6-d. Different from the simulation study, (where the theoretically maximal UPLE was set at $2 \mathrm{~mm}$ ), the maximum UPLE of the "farthest" case was $4.25 \mathrm{~mm}$ and the maximal UPLE of the "closest" case was $3.68 \mathrm{~mm}$ when 25 points were used. Since the registration points were added based on descending order of UPLE, the points with the maximum UPLE for both cases always existed when the number of points increased from 6 to 25. Although the UPLE of cadaver experiment were bigger than that of the numerical simulation, ICP-PS method still showed a more accurate result than standard ICP.

\subsection{Discussion}

In this study, we compared our proposed ICP-PS method with standard ICP in both a numerical simulation and a cadaveric experiment. The simulation approach was used to assess the effects of two basic factors on the registration accuracy: (1) the number of registration points; (2) the size of UPLE. Based on simulation and experimental results concerning registration of a femoral bone shape, our method outperformed standard ICP registration. We believe that our method provides an effective solution for the registering a known shape model to a small number of points, which aids to the potential for A-mode US based intraoperative registration in upper and lower extremity orthopedic surgeries, e.g. skeletal positioning in TKA, THA and orthopedic surgeries in elbow and should joint. The method is capable of providing reliable registration with high accuracy provided when the UPLE is low. Furthermore, we have provided a framework for estimating the required information i.e. the number of sample points for a required accuracy at a known level of error, which gives valuable information, for experimental assessments in orthopedic surgery where this type of registration is applied.

We found that, relative to the standard ICP method, our proposed registration method improved the accuracy of the final registration quite remarkably when the amplitude of UPLE was small, e.g. 0 to $1 \mathrm{~mm}$ (Figure 2.6). The added Perturbation Search was able to overcome local minima encountered by the ICP. The inclusion of a perturbation search is thus a quite powerful method to minimize the PSD value and reach a better solution. Where previously reported methods randomly perturb sample points [14,29], our method uses prior knowledge concerning the geometrical constraints imposed by the shape model representing the preoperatively known data of the patient (POM) with a more efficient search. The accuracy obtained in this simulation showed similar results to other studies[15, 20, 22, 31]. However, different accuracy metrics were used in various application scenarios and made the comparison difficult. Commonly, an error of least $1 \pm 0.3 \mathrm{~mm}$ Target Registration Error is reported. In our simulation, we found that 15 points would be required assuming a UPLE of 
Feasibility of A-mode ultrasound based intraoperative registration in computer-aided orthopedic surgery: a simulation and experimental study

$0 \mathrm{~mm}$ to achieve a RMSE error below $1 \mathrm{~mm}$. To get below $1.5 \mathrm{~mm}$ RMSE under a $1 \mathrm{~mm}$ UPLE condition, 15 points are needed on average $(1.40 \mathrm{~mm})$. As shown in Figure 2.5, compared to standard ICP, the improvement of registration accuracy of our proposed method became less effective when the UPLE increased to $[1,2] \mathrm{mm}$. This indicates that the additional steps to the standard ICP algorithm gives an improved registration result as long as the error remains sufficiently small.

The cadaver experiment results showed that the registration accuracy generally improved with an increasing number of surface sample points. However, this improvement highly depended on the error of each newly added point (see Figure 2.6-c,d). As the ICP-PS reached $2.81 \mathrm{~mm}$ for 25 points, the maximal error associated with the registration point was $3.68 \mathrm{~mm}$ (Figure 2.6-d). Therefore, only $2.81 \mathrm{~mm}$ RMSE could be achieved by ICP-PS. From these cadaver experiment results, the magnitudes of UPLE of all registration points influences the final registration accuracy. The UPLE must be carefully reduced when the ICP-PS would be applied in the clinical practice. In this study, technical feasibility has been investigated. Clinical feasibility regarding to specific surgeries (e.g. TKA, THA) would be investigated in future study.

In the simulation, the registration accuracy of our method improved more quickly than standard ICP when adding same number of points (Figure 2.5) at low UPLE. Heger et. al. performed a similar study, where they also found that the addition of palpation points on the distal femoral region improved the registration accuracy[31]. However, they used a mechanical probe to conduct pre-registration, which resulted in a more accurate initial guess than US based pre-registration.

In the zero UPLE case of the simulation results, perfect registration was possible using ICP-PS in 80 out of 100 cases using 25 sample points. Standard ICP reached perfect registration in only 13 out of 100 cases for the same amount of sample points. Whereas when using 25 sample points certain cases still could not reach the perfect registration result $(0 \mathrm{~mm}$ RMSE). Closer inspection of those cases revealed that the addition of random sample points can also lead to the inclusion of registration points with less contribution to the final result. For example, a newly added point may not anatomically be important or homogenously distributed (e.g. close to previously selected points) and that thus do not give adequately extra information for the registration. Thus, the spatial distribution of SSP must provide enough geometrical constraints for ICP-PS registration.

In this study we did not distinguish among sources of UPLE in the ultrasound data [20, 22] as all of these sources of error are difficult to quantify and measure in practice. In reality the various sources of UPLE (e.g. the inherent error of the navigation system and the point 
Feasibility of A-mode ultrasound based intraoperative registration in computer-aided orthopedic surgery: a simulation and experimental study

localization error of A-mode ultrasound) may each have slightly different effects on the registration accuracy and the overall registration accuracy will logically be differently affected. Thus, to simplify the noise distribution in simulation, we used an isotropic error model that random direction noise vector added to the SSP with uniformly distribution over a preset interval. The registration results at various UPLE levels consistently show that the registration accuracy does improve by adding more points, but it will never improve beyond a certain registration limit that is associated with the average UPLE. Hence, reducing the Ultrasound Point Localization Error in detecting the bone surface seems the most effective way to improve registration accuracy; large UPLE's cannot easily be compensated by adding more points or introducing smart perturbation algorithms to improve the registration techniques.

As we found in the results of the numerical simulation and the cadaver experiment, UPLE is a critical parameter that could affect the overall registration accuracy, especially when its magnitude is large (e.g. larger than $2 \mathrm{~mm}$ ). However, the magnitude of UPLE is difficult to quantify in clinical practice. In some cases, a tracked mechanical sensor with an optical tracking system may produce higher accuracy than a navigated A-mode US probe (influenced by thickness of soft tissue, accessibility of ultrasound waveform for different anatomical regions). Hence the proposed ICP-PS algorithm can be applied for intraoperative registration not only via an A-mode US probe but also other digitalizing sensors. To facilitate the usage of A-mode US probe in CAOS, the improvement of localization accuracy of detected ultrasound point is a necessary step toward a feasible clinical application. To improve the localization accuracy, a systematic investigation of A-mode US performance on special anatomical locations would provide valuable information regarding to ultrasound waveform accessibility and intensity of received echo, which would be a further study. The more robust and accurate calibration method should be developed in the future. To explore the actual speed of sound in soft tissue, an in-vivo experiment should be conducted to measure the actual speed of sound in the further improvement.

This study has some other limitations. Firstly, the numerical simulation we used differs from a real world situation in several ways. Nevertheless, we chose to use a simulation approach because that allowed us to assess the effects of individual parameters systematically, without interference of other parameters such as additional experimental errors and other uncertainties that will occur in an experimental setup. The optimal procedure as determined with such a numerical simulation should always be tested in in-vitro situations before proceeding to in-vivo situations (surgery). Secondly, we only compared our proposed registration algorithm with standard ICP, which is the currently the most widely used algorithm in this type of experiment. Still, further research could extend the applicability of 
Feasibility of A-mode ultrasound based intraoperative registration in computer-aided orthopedic surgery: a simulation and experimental study

our proposed simulation method to other registration algorithms for comparison (e.g. Gaussian Mixture model registration[33]), which could also be tested in the simulation framework as described in this study. Thirdly, we used an empirically designed perturbation approach based on the observation of registration results to avoid local minima. Due to the object of our registration study being a femur, which is a long cylinder-like shape, most of the 'looseness' in 3D geometry is along the distal-proximal axis. For other shapes, the principle component analysis (PCA) can be applied on the known shape model to determine the perturbation axis. The principle of avoiding local minimum of a certain registration algorithm will be equally applicable for all situations where a point cloud is needed to register to a known shape model. More suitable perturbation approaches should be investigated and designed carefully based on different purposes.

\subsection{Conclusions}

This study has presented a simulation approach to investigate the effects of the number of registration points and the Ultrasound Point Localization Error on the registration accuracy for intraoperative registration in CAOS. The finding of this simulation were then verified in a cadaver experiment. A registration algorithm using fewer points to achieve high accuracy was established and validated using numerical simulation and cadaveric experimental techniques. Furthermore, the simulation approach provides a well-defined framework for estimating the minimally required number of registration points for point cloud registration, once the required levels of accuracy and time efficiency have been set and the UPLE can be estimated. Based on the simulation and in-vitro results, the registration accuracy was shown to improve with an increasing number of registration points. The addition of a perturbation search and feedback to ICP resulted in significant improvement of accuracy for low UPLE (i.e. UPLE $<1 \mathrm{~mm}$ ) and also outperformed standard ICP during the cadaver experiment. With the high potential to implement further improvements associated with the localization accuracy of acquired registration points (i.e. UPLE), the proposed ICP-PS method has the potential to be applied on intraoperative registration of CAOS.

\section{Acknowledgements}

The authors also thank for the generous helps of Léon Driessen and Richard van Swam in cadaveric preparation from Orthopedic Research Lab, Radboud university medical center, Netherlands.

References 
Feasibility of A-mode ultrasound based intraoperative registration in computer-aided orthopedic surgery: a simulation and experimental study

1. Jenny, J.Y., E. Ciobanu, and C. Boeri, The rationale for navigated minimally invasive unicompartmental knee replacement. Clin Orthop Relat Res, 2007. 463: p. 58-62.

2. Anderson, K.C., K.C. Buehler, and D.C. Markel, Computer assisted navigation in total knee arthroplasty: comparison with conventional methods. J Arthroplasty, 2005. 20(7 Suppl 3): p. 132-8.

3. DiGioia, A.M., 3rd, et al., Mini-incision technique for total hip arthroplasty with navigation. J Arthroplasty, 2003. 18(2): p. 123-8.

4. Sikorski, J.M. and S. Chauhan, Computer-assisted orthopaedic surgery: Do we need CAOS? The Journal of Bone and Joint Surgery, 2003. 85(3): p. 319-323.

5. Amiot, L.P. and F. Poulin, Computed tomography-based navigation for hip, knee, and spine surgery. Clin Orthop Relat Res, 2004(421): p. 77-86.

6. Mavrogenis, A.F., et al., Computer-assisted navigation in orthopedic surgery. Orthopedics, 2013. 36(8): p. 631-42.

7. Maurer, C.R., Jr., R.J. Maciunas, and J.M. Fitzpatrick, Registration of head CT images to physical space using a weighted combination of points and surfaces. IEEE Trans Med Imaging, 1998. 17(5): p. 753-61.

8. Wolfsberger, S., et al., Anatomical landmarks for image registration in frameless stereotactic neuronavigation. Neurosurg Rev, 2002. 25(1-2): p. 68-72.

9. $\quad$ Caversaccio, M., et al., The "Bernese" frameless optical computer aided surgery system. Comput Aided Surg, 1999. 4(6): p. 328-34.

10. Maurer, C.R., Jr., et al., Registration of head volume images using implantable fiducial markers. IEEE Trans Med Imaging, 1997. 16(4): p. 447-62.

11. Livyatan, H., Z. Yaniv, and L. Joskowicz, Gradient-based 2-D/3-D rigid registration of fluoroscopic X-ray to CT. Medical Imaging, IEEE Transactions on, 2003. 22(11): p. 1395-1406.

12. Otake, Y., et al., Intraoperative Image-based Multiview 2D/3D Registration for Image-Guided Orthopaedic Surgery: Incorporation of Fiducial-Based C-Arm Tracking and GPU-Acceleration. Medical Imaging, IEEE Transactions on, 2012. 31(4): p. 948-962.

13. Amin, D.V., et al., Ultrasound registration of the bone surface for surgical navigation. Comput Aided Surg, 2003. 8(1): p. 1-16.

14. Chang, T.C., et al., A-Mode Ultrasound Bone Registration for Computer-Assisted Knee Surgery: Calibration and Robustness Test, in 25th Southern Biomedical Engineering Conference 2009, 15 - 17 May 2009, Miami, Florida, USA, A. McGoron, C.-Z. Li, and W.C. Lin, Editors. 2009, Springer Berlin Heidelberg. p. 97-100.

15. Fieten, L., et al., Fast and accurate registration of cranial CT images with A-mode ultrasound. Int J Comput Assist Radiol Surg, 2009. 4(3): p. 225-37. 
Feasibility of A-mode ultrasound based intraoperative registration in computer-aided orthopedic surgery: a simulation and experimental study

16. Mozes, A., et al., Three-dimensional A-mode ultrasound calibration and registration for robotic orthopaedic knee surgery. Int J Med Robot, 2010. 6(1): p. 91-101.

17. Marotti, J., et al., Recent advances of ultrasound imaging in dentistry--a review of the literature. Oral Surg Oral Med Oral Pathol Oral Radiol, 2013. 115(6): p. 819-32.

18. De Lorenzo, D., et al. Experimental validation of A-mode ultrasound acquisition system for computer assisted orthopaedic surgery. in Medical Imaging 2009: Ultrasonic Imaging and Signal Processing. 2009. Lake Buena Vista, FL.

19. Hamidzada, W.A. and E.P. Osuobeni, Agreement between A-mode and B-mode ultrasonography in the measurement of ocular distances. Vet Radiol Ultrasound, 1999. 40(5): p. 502-7.

20. Maurer, C., Jr., et al., AcouStick: A Tracked A-Mode Ultrasonography System for Registration in Image-Guided Surgery, in Medical Image Computing and Computer-Assisted Intervention - MICCAI'99, C. Taylor and A. Colchester, Editors. 1999, Springer Berlin Heidelberg. p. 953-962.

21. Popovic, A., et al., Efficient non-invasive registration with A-mode ultrasound in skull surgery. CARS 2005: Computer Assisted Radiology and Surgery, 2005. 1281: p. 821826.

22. Amstutz, C., et al., A-mode ultrasound-based registration in computer-aided surgery of the skull. Archives of Otolaryngology-Head \& Neck Surgery, 2003. 129(12): p. 1310-1316.

23. Oszwald, M., et al., Accuracy of navigated surgery of the pelvis after surface matching with an a-mode ultrasound probe. J Orthop Res, 2008. 26(6): p. 860-4.

24. Besl, P.J. and H.D. McKay, A method for registration of 3-D shapes. IEEE Transactions on Pattern Analysis and Machine Intelligence, 1992. 14(2): p. 239-256.

25. Simon, D. and T. Kanade, Geometric constraint analysis and synthesis: Methods for improving shape-based registration accuracy, in CVRMed-MRCAS'97, J. Troccaz, E. Grimson, and R. Mösges, Editors. 1997, Springer Berlin Heidelberg. p. 181-190.

26. Pellikaan, P., et al., Evaluation of a morphing based method to estimate muscle attachment sites of the lower extremity. J Biomech, 2014. 47(5): p. 1144-50.

27. Horn, B.K.P., Closed-form solution of absolute orientation using unit quaternions. Journal of the Optical Society of America A, 1987. 4(4): p. 629-642.

28. Friedman, J.H., F. Baskett, and L.J. Shustek, An Algorithm for Finding Nearest Neighbors. IEEE Transactions on Computers, 1975. C-24(10): p. 1000-1006.

29. Ma, B. and R.E. Ellis, Robust registration for computer-integrated orthopedic surgery: laboratory validation and clinical experience. Med Image Anal, 2003. 7(3): p. 23750. 
Feasibility of A-mode ultrasound based intraoperative registration in computer-aided orthopedic surgery: a simulation and experimental study

30. Azhari, H., Appendix A: Typical Acoustic Properties of Tissues, in Basics of Biomedical Ultrasound for Engineers. 2010, John Wiley \& Sons, Inc. p. 313-314.

31. Heger, S., et al., User-interactive registration of bone with A-mode ultrasound. IEEE Eng Med Biol Mag, 2005. 24(2): p. 85-95.

32. Bachler, R., H. Bunke, and L.P. Nolte, Restricted surface matching--numerical optimization and technical evaluation. Comput Aided Surg, 2001. 6(3): p. 143-52.

33. Jian, B. and B.C. Vemuri, Robust Point Set Registration Using Gaussian Mixture Models. IEEE Transactions on Pattern Analysis and Machine Intelligence, 2011. 33(8): p. 1633-1645. 

Measuring Tibiofemoral Kinematics Using One-channel 3D-Tracked AMode Ultrasound Tracking System: A Proof of Principle Study

\section{Chapter 3 Measuring Tibiofemoral Kinematics Using One-channel 3D-Tracked A-Mode Ultrasound Tracking System: A Proof of Principle Study}

The purpose of this study is to investigate the technical feasibility of measuring tibiofemoral kinematics in an in-vitro experiment by using a 3D-tracked A-mode ultrasound system and to determine its accuracy of measured kinematics. As A-mode ultrasound is capable of detecting bone surface through soft tissue in a non-invasive manner, the combination of a single A-mode ultrasound transducer with an optical motion tracking system provides the possibility for digitizing the $3 \mathrm{D}$ locations of bony points at different anatomical regions on the thigh and the shank. After measuring bony points over a large area of both the femur and tibia, the bone models of the femur and tibia that were segmented from CT or MRI images were registered to the corresponding bony points. Then the relative position of the tibia with respect to the femur could be obtained and the tibiofemoral kinematics could also be measured. A cadaveric experiment was conducted to assess the accuracy of measured tibiofemoral kinematics compared to the reference kinematics obtained by optical markers fixed to intra-cortical traction pins placed in the femur and tibia. The results showed that the ultrasound system could estimate all six degrees of freedom of tibiofemoral kinematics with $1.06 \pm 2.05^{\circ}$ (mean \pm standard deviation) error for the three rotational components and -2.16 $\pm 3.02 \mathrm{~mm}$ error for the three translational components. It was concluded that this technique is feasible and facilitates the integration of arrays of A-mode ultrasound transducers with an optical motion tracking system for non-invasive dynamic tibiofemoral kinematics measurement. 


\section{Measuring Tibiofemoral Kinematics Using One-channel 3D-Tracked A- Mode Ultrasound Tracking System: A Proof of Principle Study}

\subsection{Introduction}

Detailed knowledge of the skeletal knee kinematics is very important to assess pathologies of the lower limb [1-3]. Accurately measured tibiofemoral kinematics is also useful for evaluation of surgical techniques such as implantation of artificial knee implants $[4,5]$ and for the development and validation of computer models (e.g. musculoskeletal models) capable of simulating normal and pathological human movement $[6,7]$.

Reconstruction of three-dimensional (3D) human movement based on skin-mounted markers has become the standard procedure in clinical human motion analysis [8], where the skin-mounted markers are typically taken to represent movement of the bony segment beneath the skin. However, the spatial reconstruction of the musculoskeletal system and calculation of its kinematics via a skin marker based multi-link model are subject to Soft Tissue Artifacts (STA) [9]. The markers follow skin movement, but generate errors when used to represent motion of the underlying bony segments.

A wide variety of studies have investigated the quantification and influences of STA in the lower limb during different motor tasks [5, 10-21]. These studies found that STA were greater for the thigh than for the shank, with STA errors as high as $50 \mathrm{~mm}$ [7]. In terms of kinematics, an average error of $4.4^{\circ}$ and $13.1^{\circ}$ was found for the three rotation angles and 13.0 and $16.1 \mathrm{~mm}$ for the three translations for walking and cutting, respectively [11]. In addition, the flexion-extension rotation of the knee joint was found to be determined reliably by skin-mounted markers. However, the remaining motions in the knee joint were more severely affected by STA, which resulted in inaccuracies of relative kinematic outcomes [12]. To reduce the STA introduced by skin-mounted markers, researchers apply optimization techniques $[22,23]$ and improve the knee model based on advanced joint motion constraints [9]. Although these techniques indeed lead to globally reduced measurement errors of skin markers, the inherent mismatch between skin and bone movement is difficult to remove under all circumstances. Andersen et al. showed that the inclusion of optimized idealized knee joint constraints did not eliminate or reduce the effects of STA and did not improve the validity of the tibiofemoral kinematics derived from skin markers on the thigh and shank [16].

A method to effectively reduce STA is to utilize intra-cortical traction pins rigidly fixed to the bone and equipped with optical markers. This approach has been shown to provide a very accurate estimation of the movement of femur and tibia in the knee joint $[10,11]$. However, the invasiveness of this method severely limits its in-vivo applicability. Alternatively, fluoroscopic systems have been used to quantify joint motion in-vivo [2, 14, $15,24,25]$. Reported accuracies are in the order of $1 \mathrm{~mm}$ and 2 degrees, depending whether a dual or a single fluoroscopic system was used and whether intact knees or implants were 


\section{Measuring Tibiofemoral Kinematics Using One-channel 3D-Tracked A- Mode Ultrasound Tracking System: A Proof of Principle Study}

involved. In addition to the radiation, a drawback of the fluoroscopic systems is the limited field of view that restricts the patient's natural movement. Recently, fluoroscopic systems that are mobilized by robots which can follow the patient during gait have been proposed, allowing for more natural kinematics $[2,26]$. These types of robotized fluoroscopic systems are, however, still radiative, high in cost and workload. As such it is difficult to implement them in clinical practice on large patient cohorts.

Ultrasound (US) technology is a rapidly developing field with the advantages of noninvasiveness and non-radiation. It has become possible to register US images to the segmented bone in computer-aided orthopedic surgery [27]. The feasibility of estimating knee joint kinematics based on conventional B-mode (Brightness-mode) ultrasound transducers has also been shown [28]. As ultrasound is capable of detecting the bone boundaries through the soft tissue under dynamic motion, the combination of ultrasound technique with a motion tracking system (e.g. optical tracking system) provides a possibility to digitize the detected bone boundaries into 3D bony points. Compared to a conventional Bmode transducer, an A-mode transducer (i.e. single element ultrasound transducer) is cheaper and smaller in size and more accurate for biometric measurement, e.g. depth [29].

In this study, we developed a one-channel 3D-tracked A-mode ultrasound tracking system by combining one A-mode ultrasound transducer with optical tracking markers. The 3Dtracked A-mode ultrasound probe was used to measure bony points over a large area of both the femur and the tibia. After this measurement, the known bone models of the femur and the tibia (segmented from CT images) were registered to the corresponding bony points. Then the relative position of the tibia with respect to the femur was quantified from the position of the registered femur and the registered tibia. In the end, the tibiofemoral kinematics were measured form the relative position of the registered tibia and femur. The working principle of our proposed system is shown in Figure 3.1. Due to the fact that the position of the registered bone is reconstructed from the 3D bony points instead of skin markers, our system provides the potential to overcome the effect of STA to achieve accurate kinematic outcomes when dynamically applied. In this study, a cadaveric experiment was conducted to assess the accuracy of measured tibiofemoral kinematics compared to reference kinematics obtained by optical markers fixed to the intra-cortical traction pins placed in the femur and tibia. Hence, in this study we aimed to demonstrate the feasibility of an ultrasound tracking system applied in a static fashion. Demonstration of this feasibility of accurately reconstructing the relative positions of the tibia and femur and then measuring tibiofemoral kinematics by one-channel 3D-tracked A-mode ultrasound tracking system in this static study would point towards a level of feasibility of reconstructing tibiofemoral kinematics by combining arrays of A-mode 


\section{Measuring Tibiofemoral Kinematics Using One-channel 3D-Tracked A- Mode Ultrasound Tracking System: A Proof of Principle Study}

ultrasound transducers with an optical tracking system (i.e. multi-channel 3D-tracked Amode ultrasound tracking system) to quantify tibiofemoral kinematics in dynamic condition.

One-channel 3D-tracked A-mode ultrasound tracking system
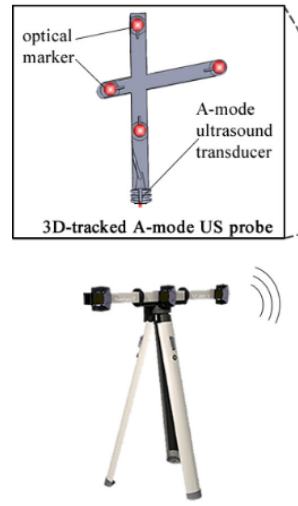

optical tracking system

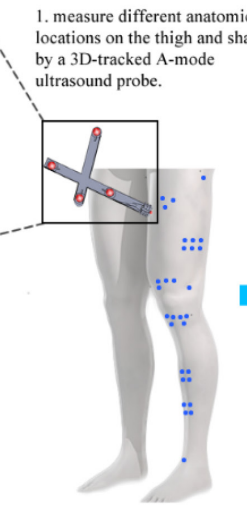

$\begin{array}{ll}\text { 1. measure different anatomical } & \text { 2. obtain bony points } \\ \text { locations on the thigh and shank } & \text { from the femur and }\end{array}$ tibia.

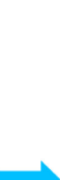

Figure 3.1 A schematic of the working principle of the one-channel A-mode ultrasound tracking system.

(I)

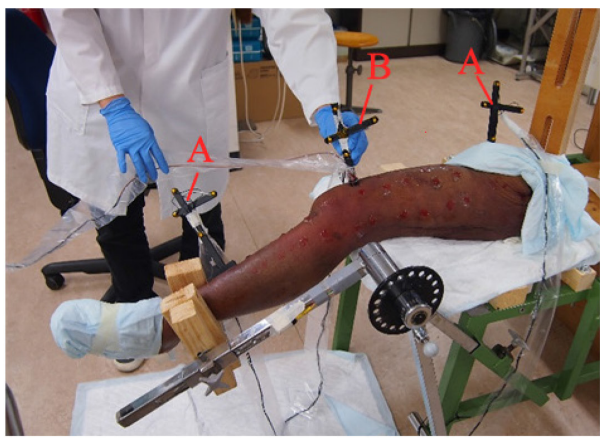

(II)

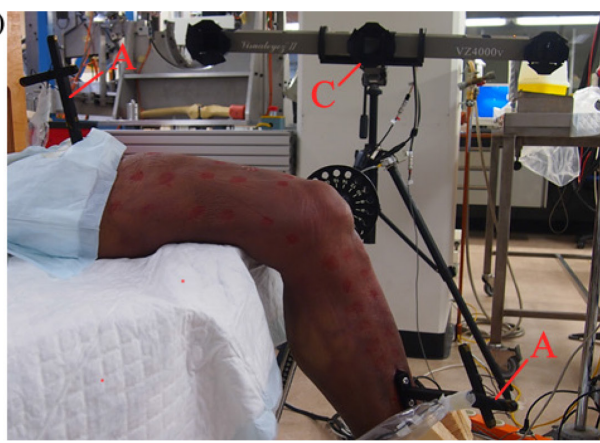

Figure 3.2 (I) Experimental setup with the cadaveric leg fixed in the rig and two intra-cortical traction pins (A) screwed into the femur and tibia separately and one 3D-tracked A-mode US probe (B) was used to measure different anatomical locations; (II) The setup maintains the stability of the leg after changing the flexion angle of the knee that is tracked by Visualeyez tracking system $(\mathrm{C})$.

\subsection{Methods}

\subsubsection{The cadaveric experimental setup}

After obtaining ethical approval, one frozen, intact left cadaveric leg (from foot to femoral head) was obtained from the anatomical department of the Radboud University Medical Center (RUNMC). After thawing, two intra-cortical traction pins were screwed into the proximal-anterior part of the femur and the middle shaft of the tibia separately, with a rigid 


\section{Measuring Tibiofemoral Kinematics Using One-channel 3D-Tracked A- Mode Ultrasound Tracking System: A Proof of Principle Study}

structure containing four optical markers that were used to record the reference motions of the bones. After mounting the intra-cortical traction pins, a CT scan was made at the Department of Radiology of the RUNMC using a TOSHIBA Aquilion ONE (TOSHIBA, Tustin, USA) with a voxel size of $0.755 \mathrm{~mm} \times 0.755 \mathrm{~mm} \times 0.500 \mathrm{~mm}$. The CT images were manually segmented and surface meshes of the femur and tibia in STL format were generated using Mimics ${ }^{\circledR} 17.0$ (Materialise N.V., Leuven, Belgium), including the 3D locations of optical markers of intra-cortical traction pins. The anatomical reference frames of the femur and tibia were defined from the generated STL Models of the femur and tibia [30]. After CT scanning, the leg was fixated in a flexion-extension rig that allowed flexion of the leg in a static manner. The femoral head was rigidly fixated with a pin thereby enabling fixation of the upper leg. The ankle was clamped tightly by wooden blocks to restrict the free movement of the shank (Figure 3.2-I). The rig could be manipulated to flex the knee. When the flexion angle of the knee was fixed at a certain angle, the setup guaranteed the stability during ultrasound measurements (Figure 3.2-II).

\subsubsection{D-tracked A-mode US probe}

A single A-mode ultrasound transducer was attached to a custom-made, crucifix-shaped probe containing four optical markers for the tracking system (Figure 3.3). The A-mode ultrasound transducer (Imasonic SAS, Voray / l'Ognon, France) had an operating frequency of $7.5 \mathrm{MHz}$ and was focused at $2.5 \mathrm{~cm}$. Two Visualeyez VZ4000v tracking systems (PTI Phoenix Technologies Inc, Vancouver, Canada) were operating at $100 \mathrm{~Hz}$ to measure the trajectories of all optical markers with less than $0.5 \mathrm{~mm}$ RMS error [31]. The ultrasound and Visualeyez systems were integrated in the Diagnostic Sonar FI Toolbox (Diagnostic Sonar Ltd., Livingston, Scotland) based on a National Instruments PXI system (National Instruments, Austin, USA) with 2.3GHZ CPU (Intel Core i7-3610QE) and 8GB RAM. The sample rate of the Diagnostic Sonar system was $40 \mathrm{MHz}$. The acquisition and post-processing software was written in LabVIEW 2014 (National Instruments, Austin, USA).

When probing the 3D-tracked A-mode US probe at different anatomical locations on the thigh and shank, both the received ultrasound signals and the $3 \mathrm{D}$ coordinates of the four optical markers were recorded to determine the $3 \mathrm{D}$ coordinates of the ultrasound reflection point (i.e. bony point) on the bone surface (Figure 3.3). To get the ultrasound reflection point, firstly the received ultrasound signal was filtered using a second-order low-pass Butterworth filter with a cut-off frequency of $2 \mathrm{MHz}$ and then a peak detection window was set manually in which a detected peak that had greater amplitude than the setting threshold was determined to be the bone surface reflection [3]. To convert the determined peak from time domain to spatial domain in distance unit, the depth $(\lambda)$ from the origin point of ultrasound beam to ultrasound reflection point was calculated using equation (1): 

Mode Ultrasound Tracking System: A Proof of Principle Study

$$
\lambda=\frac{v t}{2}
$$

where $v$ is the velocity of sound in the material $(1590 \mathrm{~m} / \mathrm{s}$ in muscle across the fibers) [32] and $t$ represents the time that ultrasound waveform takes from the origin of ultrasound beam to the bone surface and reflect back to the origin. Secondly, the origin point $\left(\vec{O}_{p}\right)$ and the unit pointing direction $\left(\vec{V}_{d i r}\right)$ of the US beam were determined from a calibration method described in [3]. Subsequently, the 3D coordinates of ultrasound reflection point $\left(\vec{R}_{p}\right)$ were calculated by:

$$
\vec{R}_{p}=\vec{O}_{p}+\lambda \vec{V}_{d i r}
$$

where $\vec{O}_{p}$ represents the original 3D coordinates of US beam and $\vec{V}_{d i r}$ represents the unit vector of the direction of US beam and $\lambda$ is the measured depth from equation (1). The abovementioned method was used to calculate ultrasound reflection points at different anatomical locations on the femur and tibia.

\subsubsection{Measurement protocol}

Since only one 3D-tracked A-mode US probe was used in this study, the cadaveric leg needed to be kept in various stationary poses so that we could obtain multiple bony points from the femur and tibia. After collecting all bony points at one stationary pose, the leg was flexed to a new position where a new set of bony points was collected. The cadaveric leg was flexed and fixed at five different positions for ultrasound measurements ranging from full extension to an approximate flexion angle of $90^{\circ}$. In addition, to ensure the bony points measured at the thigh and shank were consistently acquired at the different poses of flexion, we divided the upper and lower leg into eight regions of interest which contained Anatomical Spots (AS, see Figure 3.4) marked with red spots on the skin where we attempted to measure the bony points with the 3D-tracked A-mode US probe. A total of 28 AS were defined on the thigh and 18 AS were defined on the shank (Figure 3.4-A, B). 


\section{Measuring Tibiofemoral Kinematics Using One-channel 3D-Tracked A- Mode Ultrasound Tracking System: A Proof of Principle Study}
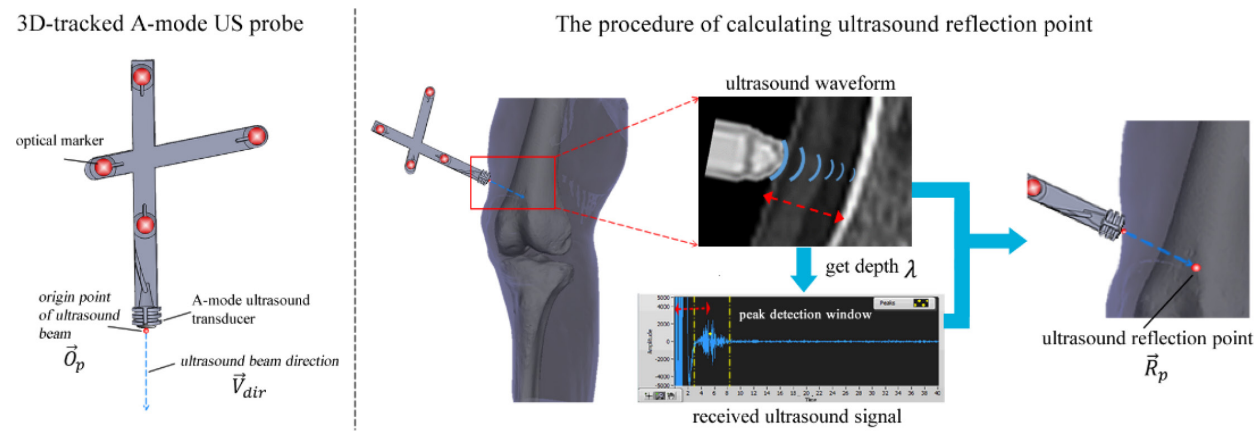

Figure 3.3 The principle of calculating the ultrasound reflection point on the bone surface via a 3D-tracked A-mode US probe. The depth $\lambda$ is determined from the received ultrasound signal by setting a peak detection window. The ultrasound reflection point $\left(\vec{R}_{p}\right)$ is determined based on the equation (2) with knowing origin point of ultrasound beam $\left(\vec{O}_{p}\right)$ and unit vector of ultrasound beam direction $\left(\vec{V}_{\text {dir }}\right)$.

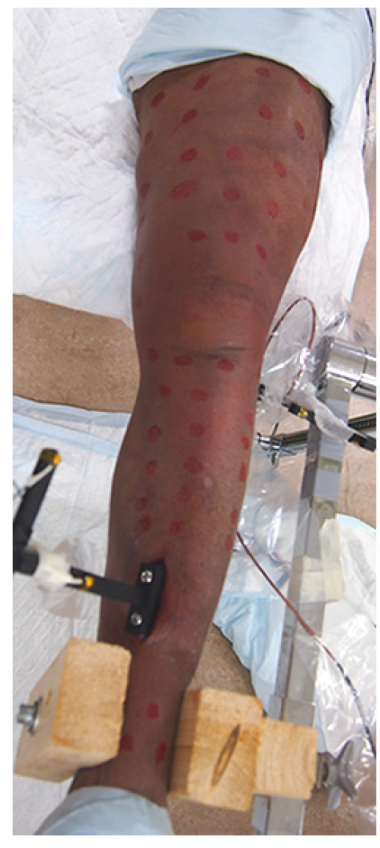

(A)

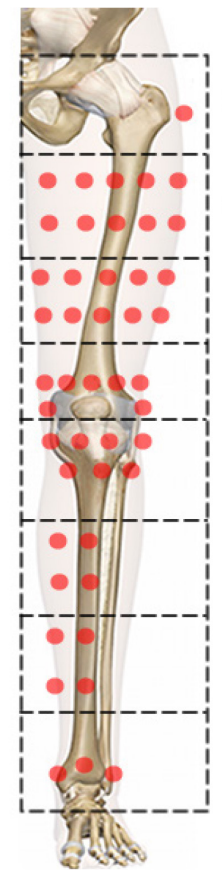

(B)

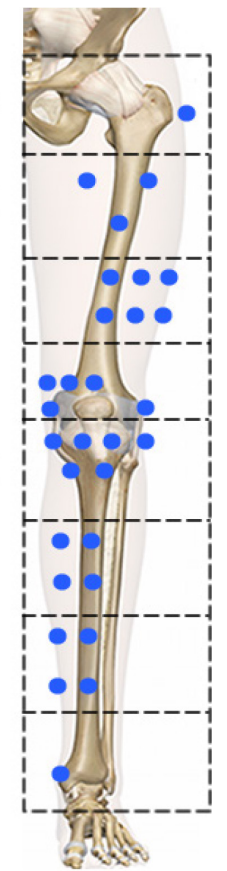

(C)

Figure 3.4 Illustration of Anatomical Spots (AS) on upper and lower leg. (A) the cadaveric leg fixed on the rig and the AS were marked in red color spots on skin; (B) the schematic of all defined AS on upper and lower leg; (C) the schematic of the finally selected AS based on anatomical importance and the ease of acquiring bone reflection points.

From a practical point of view the number of ultrasound transducers of multi-channel Amode US tracking system will be limited due to issues with limitations in electronics, cabling 


\section{Measuring Tibiofemoral Kinematics Using One-channel 3D-Tracked A- Mode Ultrasound Tracking System: A Proof of Principle Study}

and costs. Therefore, in this study, we chose to use a total of 30 ultrasound reflection points to enable calculation of the position of the tibia relative to the femur; 15 points from the femur and 15 points from the tibia. As the lateral and medial epicondyles of femur and tibia, great trochanter and ankle joint are important anatomical landmarks for lower limb intraoperative registration in orthopedic surgery $[3,33]$, we prescribed that those anatomical landmarks were to be included in selected AS. The remaining AS were selected based on the level of ease of acquiring a valid bone reflection from each AS at different flexion angles. The distribution of the finally selected AS is shown in Figure 3.4-C. When the cadaveric leg was flexed and fixed at one pose, six ultrasound measurement trials were recorded at each selected AS. Each trial was a single shot measurement. Hence, in total, $180(6 \times 30)$ bony points were measured for one fixed pose. The duration of capturing 180 bony points was about 1 hour.

\subsubsection{Estimation of the relative tibia-femoral position}

After measuring all selected AS for five flexion angles, a dataset including $900(180 \times 5)$ ultrasound reflection points was calculated. Each of the trials involved a point cloud consisting of 30 bony points from the femur and tibia. In the registration phase, the Original Bone Models (termed OBM) of the femur and tibia were registered to those 30 bony points and finally the most likely position of the tibia relative to the femur was calculated and tibiofemoral kinematics can be quantified from the relative positions of the Registered Bone Models (termed, RBM).

The Iterative Closest Points (ICP) algorithm[34] provides an efficient method to compute the transformation between the point cloud and the OBM. To avoid local minima of the ICP registration, a perturbation method was employed to search for another possible solution around the result of first ICP iteration. The perturbation was implemented as a rotation of RBM around its distal-proximal axis from -5 to 5 degrees with intervals of 1 degree and a translation along the same axis from -3 to $3 \mathrm{~mm}$ with intervals of $0.5 \mathrm{~mm}$. If the perturbation method produced a more optimal result (i.e. smaller differences between the distance of point cloud and the RBM) than the first previous ICP iteration, the same ICP algorithm was repeated from the position provided by the perturbation. An iterative loop that included perturbation, followed by the ICP was then repeated until convergence (i.e. perturbation did not find a more optimal result), with a maximum of 5 iterations. After registration, the relative position of the registered tibia respect to the femur was used to measure the tibiofemoral kinematics using the method described by Grood and Suntay [35]. The measured kinematics were compared to the reference tibiofemoral kinematics which were derived utilizing the 3D locations of intra-cortical traction pins on the femur and tibia using a point-to-point 
Measuring Tibiofemoral Kinematics Using One-channel 3D-Tracked AMode Ultrasound Tracking System: A Proof of Principle Study

registration algorithm[36] at the different poses. Using the selected AS (Figure 3.4-C), the registration algorithm was employed in 6 consecutive trials for every pose ( 5 poses in total).

\subsection{Results}

For flexion(+)/extension(-) and adduction(+)/abduction(-) rotations, the mean error were sub-degree compared with reference tibiofemoral kinematics (Figure 3.5; Table 3.1). RootMean-Square (RMS) errors ranged from $0.95^{\circ}$ to $3.30^{\circ}$ for joint rotations and ranged from $3.20 \mathrm{~mm}$ to $4.20 \mathrm{~mm}$ for joint translations. The largest rotational error was associated with external-internal rotation whereas the anterior-posterior translation produced the largest translational error. The overall mean and standard deviation (SD) was $1.06 \pm 2.05^{\circ}$ (mean \pm $\mathrm{SD})$ for three rotational components and $-2.16 \pm 3.02 \mathrm{~mm}$ (mean $\pm \mathrm{SD}$ ) for three translation components.

Table 3.1 Mean, Standard Deviation (SD) and Root-Mean-Square (RMS) errors across six US trials for relative tibiofemoral kinematics compared to reference kinematics: Flexion-Extension (Flex/Ext), Adduction-Abduction (Add/Abd), External-Internal (Ext/Int) rotations; Anterior-Posterior (Ant/Post), Proximal-Distal (Prox/Dist) and Lateral-Medial (Lat/Med) translations.

\begin{tabular}{lllllll}
\hline \hline & \multicolumn{2}{l}{ Joint rotational errors $\left(^{\circ}\right)$} & & \multicolumn{2}{c}{ Joint translational errors (mm) } \\
\cline { 2 - 7 } & Flex/Ext & Add/Abd & Ext/Int & Ant/Post & Prox/Dist & Lat/Med \\
\hline Mean & 0.49 & 0.85 & 1.85 & -2.22 & -2.80 & -1.44 \\
SD & 0.83 & 1.86 & 2.78 & 3.62 & 2.35 & 2.90 \\
RMS & 0.95 & 2.01 & 3.30 & 4.20 & 3.63 & 3.20 \\
\hline \hline
\end{tabular}




\section{Measuring Tibiofemoral Kinematics Using One-channel 3D-Tracked A- Mode Ultrasound Tracking System: A Proof of Principle Study}
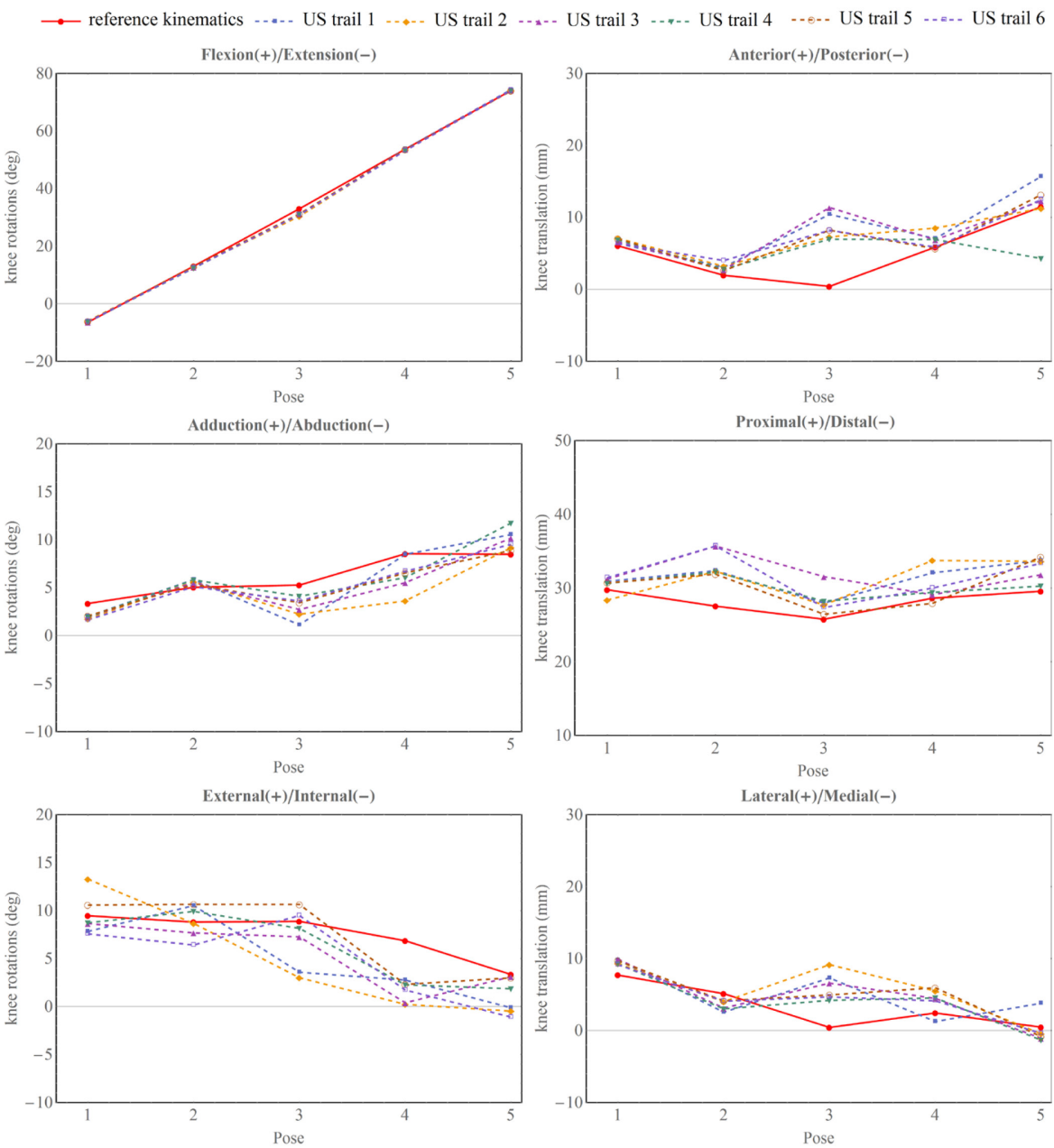

Figure 3.5 Comparisons of the six US trials of ultrasound determined tibiofemoral kinematics (dashed lines) and the reference kinematics (red, solid line) on knee joint flexion(+)/extension(-), adduction(+)/abduction(-) and external(+)/internal(-) rotations and anterior(+)/posterior(-), proximal $(+) / \operatorname{distal}(-)$ and lateral $(+) /$ medial(-) translations.

\subsection{Discussion:}

This study has shown that a one-channel 3D-tracked A-mode US tracking system can measure tibiofemoral kinematics in a static fashion with an accuracy of $1.06 \pm 2.05^{\circ}$ (mean $\pm \mathrm{SD}$ ) for rotations and $-2.16 \pm 3.02 \mathrm{~mm}$ (mean $\pm \mathrm{SD}$ ) for translations. A rather unique feature of this system is the combination of an A-mode ultrasound transducer with a conventional motion tracking system (optical tracking system in this study) to measure the trajectories of bony points instead of markers mounted on the skin. Subsequently, the registration method was applied to the acquired cloud of bony points to estimate the relative position of the tibia 


\section{Measuring Tibiofemoral Kinematics Using One-channel 3D-Tracked A- Mode Ultrasound Tracking System: A Proof of Principle Study}

with respect to the femur after which the tibiofemoral kinematics could be obtained. In this study we used a cadaver experiment to demonstrate its technical feasibility. With this study the technique has shown the potential to be extended to a multi-channel 3D-tracked A-mode ultrasound tracking system for measuring dynamic movements. Dynamic tibiofemoral kinematic measurements in $3 \mathrm{D}$ space require the determination of the instantaneous positon and orientation of the femur and tibia[37]. This study demonstrates the feasibility of such system. However, to complete such a system, a number of issues involved in dynamically measuring bony points and estimating the position and orientation of bone under dynamic conditions will be investigated in the future studies.

In contrast to the utilization of skin-mounted marker systems where STA (i.e. differences between marker and bone positions) are generally existed and are difficult to eliminate its effects [37,38], we utilized the measured ultrasound reflection points to localize bone surface in order to estimate the position and orientation of underlying bone [3], which has the potential to overcome the effect of STA. Therefore, this technique has the potential to be applied in gait analysis studies. However, in this paper, we did not perform a direct comparison with a skin marker system. To investigate this, a multi-channel 3D-tracked Amode ultrasound tracking system is necessary for simultaneous ultrasound and skin marker measurements.

Relative to our concept, the utilization of a fluoroscopy system to estimate the position and orientation of bone is different, since it relies on the radiological images and adequate model-based techniques [24, 39, 40]. Fluoroscopy systems provide highly accurate measurements of tibiofemoral kinematics, especially for knee with implants [26, 39]. Guan et al. reported that maximum root-mean-squared errors were $0.33 \mathrm{~mm}$ and $0.65^{\circ}$ for translations and rotations of the TKA knee and $0.78 \mathrm{~mm}$ and $0.77^{\circ}$ for translations and rotations of the intact knee [26]. The maximum root-mean-squared errors were $4.20 \mathrm{~mm}$ and $3.30^{\circ}$ for translations and rotations of the intact knee for our one-channel ultrasound system, which is much less accurate than a biplane fluoroscopy system. However, relative to fluoroscopic systems, the advantages of our system are the larger field of view (similarly as conventional skin marker systems) and the lack of radiation.

We defined the desired accuracies of a multi-channel 3D-tracked A-mode ultrasound tracking system to be less than $1 \mathrm{~mm}$ and $1^{\circ}$ for translational and rotational errors, respectively. Thereby we would be close the accuracy of a biplanar fluoroscopic system. For the one-channel US system as demonstrated in this study, the accuracy of measured tibiofemoral kinematics could achieve sub-degree mean errors for flexion-extension and adduction-abduction. However, external-internal rotation produced the largest mean error 


\section{Measuring Tibiofemoral Kinematics Using One-channel 3D-Tracked A- Mode Ultrasound Tracking System: A Proof of Principle Study}

$\left(1.85^{\circ}\right)$ for rotations and the translational mean errors ranged from $-1.45 \mathrm{~mm}$ to $-2.80 \mathrm{~mm}$. Hence, in its current form the system does not fulfill the requirements yet.

Considering the working principle of this technique, there are two types of errors caused inaccurate measurements of tibiofemoral kinematics: (1) the extrinsic errors in detection of a bony point; (2) intrinsic errors of registration algorithm. The extrinsic errors in the bony point detection mainly stem from the detection of the wrong peak caused by the problem of distinguishing between real and spurious peaks amongst the noisy ultrasound receiving signal. To mitigate this type of error, more advanced and robust peak detection methods can be developed. The intrinsic errors were caused by the fact that both the femur and tibia are rather tubular in shape. Hence, a small number of points on the tubular surface do not provide sufficient constraints on external-internal rotation and proximal-distal translation. As the bony points located on the two ends of bone (e.g. condyles of femur, femoral head) are inaccessible for the ultrasound beam, the registration method gives relative weak constraints for external-internal rotation and proximal-distal translation and also is susceptible to be stuck in a local minimum. More robust registration algorithm can be developed to tackle this specific registration problem in the future.

This study has several limitations. Firstly, we used only one cadaver leg in the experiment and more cadaver legs could provide more robustness information regarding the differences of geometry of bony segment and the thickness of soft tissue. Secondly, we put the cadaver leg in a rig and moved the leg in different static poses. Therefore, there was no dynamic muscle activation and the soft tissues may have moved differently relative to the bone than under in-vivo conditions. This may influence the expected accuracy of the US signal when used under in-vivo conditions. Future studies will focus on dynamically measuring ultrasound signals and investigating the way to fix the 3D-tracked A-mode US probe for different anatomical locations. In future studies special ultrasound holders that are customized for different anatomical regions will be one of the solutions to combine optical markers and arrays of A-mode ultrasound transducers for detecting reliable bony points. Thirdly, no direct comparison with a skin marker system and a fluoroscopic system was included in this study, preventing quantitative comparison between the systems.

Obviously, this study also has strengths. Firstly, it focused on investigating the feasibility of this system. The experiment was conducted in a highly controlled manner with navigated intra-cortical traction pins as ground truth. This allowed for the best way to define the errors of the US system.

Secondly, we used a single A-mode US system and applied it to static positions of the knee joint. By using this method we found a cheap and fast way to assess the potential of a 
Measuring Tibiofemoral Kinematics Using One-channel 3D-Tracked AMode Ultrasound Tracking System: A Proof of Principle Study

multi-channel system without actually acquiring all equipment and developing all software integrations.

The results from this study, demonstrating the efficacy of a single A-mode transducer, can be extended to a multiple transducers system that maybe create a new approach of noninvasive measurement of tibiofemoral kinematics in gait analysis and detailed prosthetic measurement. Further research needs to be directed to implementing the multi-channel 3Dtracked A-mode ultrasound tracking system. In-vitro and in-vivo experiments will need to be conducted to validate the multi-channel A-mode US tracking system under dynamic circumstances.

\subsection{Conclusion}

This study has presented a one-channel 3D-tracked A-mode ultrasound tracking system and proven its feasibility for measuring tibiofemoral kinematics in a cadaveric experiment. Our proposed 3D-tracked A-mode ultrasound system could achieve a mean of $1.06 \pm 2.05^{\circ}$ and $-2.16 \pm 3.02 \mathrm{~mm}$ error for rotational components and translational components, respectively. Although there are opportunities to further reduce the errors, this study already demonstrates the feasibility of extending it to a multi-channel A-mode ultrasound tracking system for measuring tibiofemoral kinematics under dynamic conditions.

\section{Acknowledgements}

The authors also thank for the generous helps of Léon Driessen and Richard van Swam in cadaveric preparation from Orthopedic Research Lab, Radboud university medical center, Netherlands.

\section{References}

1. Reinschmidt, C., et al., Tibiofemoral and tibiocalcaneal motion during walking: external vs. skeletal markers. Gait \& Posture, 1997. 6(2): p. 98-109.

2. Dennis, D.A., et al., In vivo determination of normal and anterior cruciate ligamentdeficient knee kinematics. Journal of Biomechanics, 2005. 38(2): p. 241-253.

3. Mozes, A., et al., Three-dimensional A-mode ultrasound calibration and registration for robotic orthopaedic knee surgery. Int J Med Robot, 2010. 6(1): p. 91-101.

4. Banks, S.A. and W.A. Hodge, Accurate measurement of three-dimensional knee replacement kinematics using single-plane fluoroscopy. Biomedical Engineering, IEEE Transactions on, 1996. 43(6): p. 638-649. 


\section{Measuring Tibiofemoral Kinematics Using One-channel 3D-Tracked A- Mode Ultrasound Tracking System: A Proof of Principle Study}

5. Lin, C.-C., et al., Effects of soft tissue artifacts on differentiating kinematic differences between natural and replaced knee joints during functional activity. Gait \& Posture, 2016. 46: p. 154-160.

6. Pandy, M.G., Computer modeling and simulation of human movement. Annu Rev Biomed Eng, 2001. 3: p. 245-73.

7. Akbarshahi, M., et al., Non-invasive assessment of soft-tissue artifact and its effect on knee joint kinematics during functional activity. Journal of Biomechanics, 2010. 43(7): p. 1292-1301.

8. Cappozzo, A., et al., Human movement analysis using stereophotogrammetry: Part 1: theoretical background. Gait \& Posture, 2005. 21(2): p. 186-196.

9. Lu, T.W. and J.J. O'Connor, Bone position estimation from skin marker coordinates using global optimisation with joint constraints. Journal of Biomechanics, 1999. 32(2): p. 129-134.

10. Ramsey, D.K. and P.F. Wretenberg, Biomechanics of the knee: methodological considerations in the in vivo kinematic analysis of the tibiofemoral and patellofemoral joint. Clinical Biomechanics, 1999. 14(9): p. 595-611.

11. Benoit, D.L., et al., Effect of skin movement artifact on knee kinematics during gait and cutting motions measured in vivo. Gait \& Posture, 2006. 24(2): p. 152-164.

12. Leardini, A., et al., Human movement analysis using stereophotogrammetry: Part 3. Soft tissue artifact assessment and compensation. Gait \& Posture, 2005. 21(2): p. 212-225.

13. Peters, A., et al., Quantification of soft tissue artifact in lower limb human motion analysis: A systematic review. Gait \& Posture, 2010. 31(1): p. 1-8.

14. Barr, A., et al., Soft Tissue Artifact Assessment During Treadmill Walking in Subjects With Total Knee Arthroplasty. IEEE Transactions on Biomedical Engineering, 2013. 60(11): p. 3131-3140.

15. Garling, E.H., et al., Soft-tissue artefact assessment during step-up using fluoroscopy and skin-mounted markers. J Biomech, 2007. 40 Suppl 1: p. S18-24.

16. Andersen, M.S., et al., Do kinematic models reduce the effects of soft tissue artefacts in skin marker-based motion analysis? An in vivo study of knee kinematics. Journal of Biomechanics, 2010. 43(2): p. 268-273.

17. Benoit, D.L., M. Damsgaard, and M.S. Andersen, Surface marker cluster translation, rotation, scaling and deformation: Their contribution to soft tissue artefact and impact on knee joint kinematics. Journal of Biomechanics, 2015. 48(10): p. 2124-2129.

18. Camomilla, V., et al., Non-invasive assessment of superficial soft tissue local displacements during movement: A feasibility study. Journal of Biomechanics, 2009. 42(7): p. 931-937. 


\section{Measuring Tibiofemoral Kinematics Using One-channel 3D-Tracked A- Mode Ultrasound Tracking System: A Proof of Principle Study}

19. Clément, J., et al., Soft tissue artifact compensation in knee kinematics by multibody optimization: Performance of subject-specific knee joint models. Journal of Biomechanics, 2015. 48(14): p. 3796-3802.

20. Freeman, M.A.R. and V. Pinskerova, The movement of the normal tibio-femoral joint. Journal of Biomechanics, 2005. 38(2): p. 197-208.

21. Miranda, D.L., et al., Kinematic differences between optical motion capture and biplanar videoradiography during a jump-cut maneuver. Journal of Biomechanics, 2013. 46(3): p. 567-573.

22. Andriacchi, T.P., et al., A point cluster method for in vivo motion analysis: applied to a study of knee kinematics. J Biomech Eng, 1998. 120(6): p. 743-9.

23. Garling, E.H., et al., Marker Configuration Model-Based Roentgen Fluoroscopic Analysis. Journal of Biomechanics, 2005. 38(4): p. 893-901.

24. Anderst, W., et al., Validation of Three-Dimensional Model-Based Tibio-Femoral Tracking During Running. Medical engineering \& physics, 2009. 31(1): p. 10-16.

25. You, B.M., et al., In vivo measurement of 3-D skeletal kinematics from sequences of biplane radiographs: Application to knee kinematics. IEEE Transactions on Medical Imaging, 2001. 20(6): p. 514-525.

26. Guan, S., et al., Mobile Biplane X-Ray Imaging System for Measuring 3D Dynamic Joint Motion During Overground Gait. IEEE Trans Med Imaging, 2016. 35(1): p. 326-36.

27. Barratt, D.C., et al., Instantiation and registration of statistical shape models of the femur and pelvis using 3D ultrasound imaging. Medical Image Analysis, 2008. 12(3): p. 358374.

28. Masum, M.A., et al., Accuracy assessment of Tri-plane B-mode ultrasound for noninvasive 3D kinematic analysis of knee joints. Biomed Eng Online, 2014. 13: p. 122.

29. Hamidzada, W.A. and E.P. Osuobeni, Agreement between A-mode and B-mode ultrasonography in the measurement of ocular distances. Vet Radiol Ultrasound, 1999. 40(5): p. 502-7.

30. Miranda, D.L., et al., Automatic determination of anatomical coordinate systems for three-dimensional bone models of the isolated human knee. Journal of Biomechanics, 2010. 43(8): p. 1623-1626.

31. Inc., P.P.T. VZ4000v technical specifications. [cited 20173 Mar]; Available from: http://www.ptiphoenix.com/products/trackers/VZ4000v.

32. Azhari, H., Appendix A: Typical Acoustic Properties of Tissues, in Basics of Biomedical Ultrasound for Engineers. 2010, John Wiley \& Sons, Inc. p. 313-314.

33. Barratt, D.C., et al., Self-calibrating 3D-ultrasound-based bone registration for minimally invasive orthopedic surgery. IEEE Trans Med Imaging, 2006. 25(3): p. 312-23. 


\section{Measuring Tibiofemoral Kinematics Using One-channel 3D-Tracked A- Mode Ultrasound Tracking System: A Proof of Principle Study}

34. Besl, P.J. and H.D. McKay, A method for registration of 3-D shapes. IEEE Transactions on Pattern Analysis and Machine Intelligence, 1992. 14(2): p. 239-256.

35. Grood, E.S. and W.J. Suntay, A joint coordinate system for the clinical description of three-dimensional motions: application to the knee. J Biomech Eng, 1983. 105(2): p. 13644 .

36. Horn, B.K.P., Closed-form solution of absolute orientation using unit quaternions. Journal of the Optical Society of America A, 1987. 4(4): p. 629-642.

37. Cappozzo, A., et al., Position and orientation in space of bones during movement: experimental artefacts. Clinical Biomechanics, 1996. 11(2): p. 90-100.

38. Cappozzo, A., et al., Position and orientation in space of bones during movement: anatomical frame definition and determination. Clin Biomech (Bristol, Avon), 1995. 10(4): p. 171-178.

39. Bingham, J. and G. Li, An Optimized Image Matching Method for Determining InVivo TKA Kinematics with a Dual-Orthogonal Fluoroscopic Imaging System. Journal of Biomechanical Engineering, 2006. 128(4): p. 588-595.

40. Giphart, J.E., et al., Accuracy of a contour-based biplane fluoroscopy technique for tracking knee joint kinematics of different speeds. Journal of Biomechanics, 2012. 45(16): p. 2935-2938. 


\section{Chapter 4 A Novel Multi-channel 3D-Tracked A- Mode Ultrasound System to Measure Tibiofemoral Kinematics}

Objective: This paper presents a novel method to measure 3-D tibiofemoral kinematics using a multi-channel A-mode ultrasound system which is integrated with a conventional motion capture system (i.e. optical tracking system). Utilizing this system, the 6 degrees of freedom (DOF) of the tibiofemoral kinematics can be quantified in a non-invasive and non-radiative manner. Methods: In this study we demonstrate its feasibility and quantify its achievable accuracy in a cadaveric setting. During the experiments, the knee joint was manually moved into flexion and extension. Six customized ultrasound holders containing a total of $30 \mathrm{~A}-$ mode ultrasound transducers and 18 optical markers were mounted on different anatomical regions of the lower extremity of the specimen to quantify ultrasound derived tibiofemoral kinematics. Tracked intra-cortical bone pins were inserted into the femur and tibia to quantify tibiofemoral kinematics as gold standard. Results: Average rotational errors of $1.51 \pm 1.13^{\circ}$ (mean \pm standard deviation) and translational errors of $3.14 \pm 1.72 \mathrm{~mm}$ were obtained for the ultrasound derived tibiofemoral kinematics, relative to gold standard kinematics. Conclusion: This multi-channel A-mode ultrasound system is capable of measuring tibiofemoral kinematics under dynamic motion. Significance: With the advantages of relative high accuracy, non-invasiveness, and non-radiation, this method has the potential to be adopted into gait analysis and prosthetic kinematic measurements to obtain high accuracy of 6 DOF tibiofemoral kinematics. 


\section{A Novel Multi-channel 3D-Tracked A-Mode Ultrasound System to Measure Tibiofemoral Kinematics}

\subsection{INTRODUCTION}

Measuring joint kinematics during daily activities is important for understanding normal and pathological joint functions and for assessing the outcome of orthopedic surgery and how this is potentially related to designs of orthopedic joint implants. In order to accurately determine the skeletal kinematics the acquisition of reliable data representing bone motion is essential [1]. At the knee joint, accurate tibiofemoral kinematics have been obtained so that the clinician can fully understand, diagnose and evaluate the behavior of the intact, diseased or artificial knee.

To measure in vivo kinematics of the lower extremity several researchers have used intracortical bone pins with mounted markers [1-3]. The positions of the inserted bone pins are measured by a stereo photographic system (e.g. optical tracking system). This method provides a very accurate representation of the motion of bone segments in the knee joint. However, the invasiveness of this method impedes its application into clinical practice. As an alternative, utilization of skin-mounted markers is currently the most widely used method for measuring kinematics of the lower extremity for gait analysis, in which the trajectories of skin-mounted markers represent the movements of the bony segments beneath the skin [4-7]. However, this method is limited by its accuracy of estimated kinematics, which is subject to Soft Tissue Artifacts (STA), with root mean square errors as high as $29.3 \mathrm{~mm}$ [8]. In term of kinematics, an average error of $4.4^{\circ}$ and $13.1^{\circ}$ was found for rotational angles and 13.0 and $16.1 \mathrm{~mm}$ for translations for walking and cutting activities, respectively [3]. Alternatively, fluoroscopic systems utilize the radiographic images and adequate model based methods [912] to be able to achieve accuracies of kinematics in the order of $1 \mathrm{~mm}$ for translations and 2 degrees for rotations [13-15], but induced irradiation to the subject and a limited field of view and its high cost hamper routine usage in the clinical setting.

Ultrasound (US) technology provides the possibility of detecting the tissue-bone boundary and estimating its depth through the soft tissue during movement with the advantages of noninvasiveness and non-radiation. It also is possible to register ultrasound images to the segmented bone in computer-aided orthopedic surgery [16-18]. A tri-plane B-mode (Brightness-mode) ultrasound based method demonstrated the feasibility of estimating knee joint kinematics through combing multiple B-mode ultrasound transducers and motion sensors and employing image registration algorithm[19]. Compared to a B-mode transducer, an A-mode transducer (i.e. single element ultrasound transducer) is cheaper and smaller in size and more accurate for biometric measurement, e.g. depth [20].

In this study we developed a novel tibiofemoral kinematics measurement system based on multiple A-mode ultrasound transducers integrated with a conventional motion capture 


\section{A Novel Multi-channel 3D-Tracked A-Mode Ultrasound System to Measure}

Tibiofemoral Kinematics

system (i.e. optical tracking system) to track knee joint movement. The ultrasound transducers ensured continuous sight of 3D spatial locations of the femur and tibia under dynamic conditions. Multiple customized ultrasound holders were designed to attach to six anatomical regions on the lower extremity, thereby digitizing different bone refection points as the subject moved. Digitized bone reflection points were used to reconstruct 3D relative position of the femur with respect to the tibia. The aim of this paper is twofold: first, to describe the methods developed for measuring tibiofemoral kinematics, including data acquisition, signal processing and tibiofemoral pose estimation; and second, to prove the feasibility of our proposed method and to determine the accuracy of measured tibiofemoral kinematics. A cadaveric experiment was performed and the kinematics as obtained by the new system were compared to those obtained by utilizing intra-cortical bone pins which were tracked with a motion capture system.

\subsection{System design and methods}

\subsubsection{Data Acquisition}

The multi-channel 3D-tracked A-mode ultrasound tracking system we developed comprises of an optical tracking system (VZ4000v tracking systems, PTI Phoenix Technologies Inc, Vancouver, Canada) and six customized 3D-tracked ultrasound holders (Figure 4.1). Each 3D-tracked ultrasound holder contains three optical markers that provide spatial localization information and different numbers of A-mode ultrasound transducers (Imasonic SAS, Voray / l'Ognon, France) to detect the depth of tissue-bone boundary. In total, 30 A-mode ultrasound transducers and 18 optical markers were used in the six ultrasound holders. The transducers had an operating frequency of $7.5 \mathrm{MHz}$ and were focused at $2.5 \mathrm{~cm}$. The 3D-tracked ultrasound holders were designed in SolidWorks 2016 (Waltham, Massachusetts, USA) based on the anatomical dimensions of a subject in order to ensure good skin contact of every A-mode ultrasound transducer. The designed ultrasound holders were manufactured by 3D printing device (EOS Formiga P110, EOS GmbH, Krailling, Germany) using Polyamide powder material. As a consequence of the high accuracy of the $3 \mathrm{D}$ printing process and the highly rigid design of the holders, the relative spatial relations between the three optical markers and the A-mode ultrasound transducers remained constant during all measurements. For each A-mode ultrasound transducer, the origin point $\left(\vec{O}_{p}\right)$ and the unit pointing direction $\left(\vec{V}_{\text {dir }}\right)$ of the ultrasound beam was calculated in the local holder coordinate system $\left(\mathrm{Local}_{C S}\right)$ during the holder design process. In addition, the 3D locations of the three optical markers ( marker $_{\text {Local }}$ ) were also digitized in the Local $_{C S}$ for each 3Dtracked ultrasound holder. During measurement, the 3D locations of the three optical markers for each ultrasound holder were recorded concurrently in the global coordinate system 


\section{A Novel Multi-channel 3D-Tracked A-Mode Ultrasound System to Measure} Tibiofemoral Kinematics

$\left(\right.$ global $\left._{C S}\right)$, termed markers global $_{\text {. The rigid-body transformation from markers }}$ Local $_{\text {to }}$ markers $_{\text {global }}$ of each ultrasound holder was found using point-to-point registration method described in [21], termed $T_{R E G}$. Hence, in this way, an ultrasound refection point $\left(\vec{R}_{p}\right)$ could be digitized when the depths $(\lambda)$ were obtained from the received ultrasound signals (Figure 4.1).

$$
\vec{R}_{p}=T_{R E G}\left(\vec{O}_{p}+\lambda \vec{V}_{d i r}\right)
$$
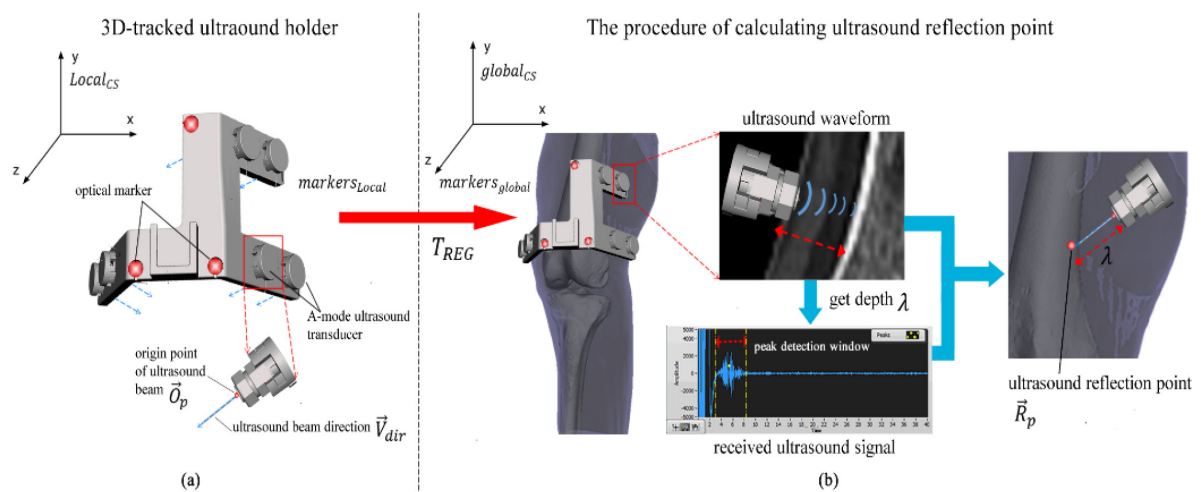

Figure 4.1 The principle of calculating the ultrasound reflection point on the bone surface via a 3D-tracked ultrasound holder. The depth $\lambda$ is determined from the received ultrasound signal by setting a peak detection window. The ultrasound reflection point $\left(\vec{R}_{p}\right)$ was determined based on obtaining the origin point of the ultrasound beam $\left(\vec{O}_{p}\right)$ and the unit vector of the ultrasound beam direction $\left(\vec{V}_{\text {dir }}\right)$ and the transformation $\left(T_{R E G}\right)$ that matches three optical markers from the local holder coordinate system to the global coordinate system.

Multi-channel 3D-tracked A-mode ultrasound tracking system

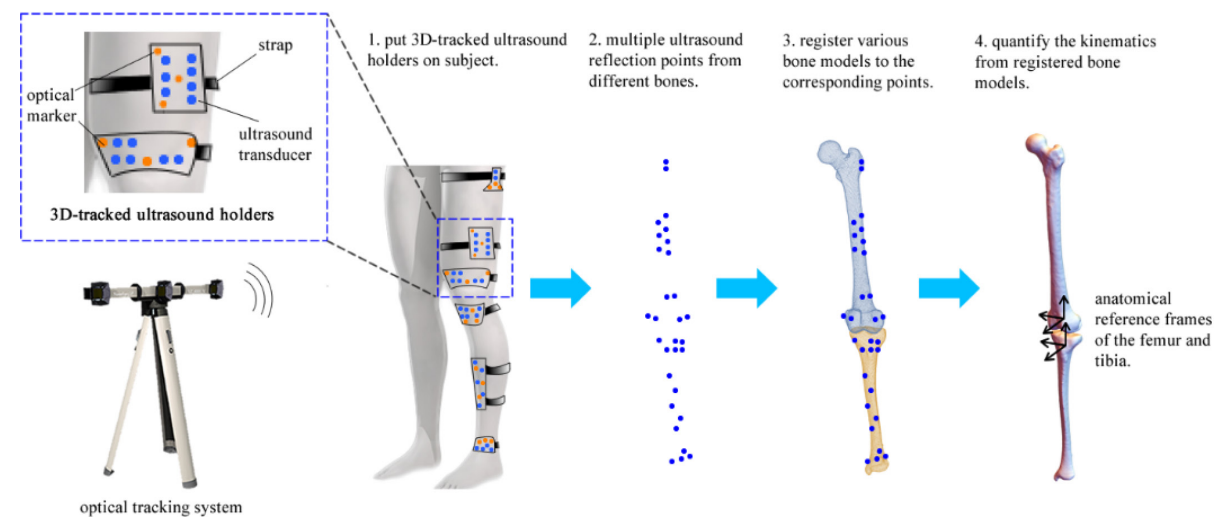

Figure 4.2 A schematic representation of the multi-channel A-mode ultrasound system to quantify tibiofemoral kinematics. 


\section{A Novel Multi-channel 3D-Tracked A-Mode Ultrasound System to Measure}

Tibiofemoral Kinematics

\subsubsection{Working Principle}

The working principle of the multi-channel 3D-tracked A-mode ultrasound tracking system is shown in Figure 4.2. In such a system, six customized 3D-tracked ultrasound holders are attached to six corresponding anatomical areas including greater trochanter, middle part of femur, distal femur, proximal tibia, middle part of tibia and ankle. These 3Dtracked ultrasound holders are tracked by an optical tracking system concurrently. The multiple ultrasound reflection points from the femur and tibia are digitized as the subject moves. Subsequently segmented bone models of the tibia and femur obtained from CT are instantaneously registered to the corresponding ultrasound reflection points using registration algorithms [22-24]. Eventually, the kinematics of the knee joint can be derived from the positions of the registered bone models. In this way the relative positions of the registered femur and the registered tibia can be monitored allowing for the quantification of rotations and translations of the two segments relative to each other during dynamic motion (Figure $4.2)$.

\subsubsection{Ultrasound Signal Processing}

The received ultrasound signal of each A-mode ultrasound transducer was filtered using a second-order low-pass Butterworth filter with a cut-off frequency of $2 \mathrm{MHz}$. Before dynamic measurements, a specific peak detection window for each transducer was manually set in the received ultrasound waveform based on the ultrasound signal obtained during flexing the knee joint of the subject in advance. The transducer-specific window allowed for a faster and more reliable peak detection in the most likely range of occurrence of the tissue-bone boundary at the different transducer locations. During the dynamic measurements, a peak detection method that finds a greater amplitude than the setting threshold and also with the biggest slope was used to determine the peak representing tissue-bone boundary.

\subsubsection{Tibiofemoral Pose Estimation}

After attaching all ultrasound holders on the lower extremity, 30 ultrasound reflection points could be digitized concurrently as the subject moved. All obtained ultrasound reflection points were fed to a registration algorithm that combined an iterative closest point algorithm [25] and perturbation method [23] to register bony segments to corresponding ultrasound reflection points per time-frame. The registration was performed by providing an initial guess of the pose of an object and following an optimization that minimizes the cost function of point-to-object matching. In our case, we used several anatomical landmarks to obtain an initial guess (pre-registration), including the lateral/medial femoral epicondyles and tibia epicondyles, greater trochanter and ankle. A subsequent optimization was performed by a modified weighted iterative closest point algorithm [26, 27]. The weighting factors were 


\section{A Novel Multi-channel 3D-Tracked A-Mode Ultrasound System to Measure Tibiofemoral Kinematics}

associated with the intensity (i.e. 'reliability') of the detected peak of the tissue-bone boundary. The registration algorithm produced the transformation matrix that matched the segmented bone model to the acquired ultrasound refection points. After applying the calculated transformation matrix to the segmented bone model, the estimated 3D position and orientation of bone model were obtained (i.e. the position of registered bone model). When the anatomical coordinate systems of the femur and tibia were determined from the segmented bone models [28], the tibiofemoral kinematics could be calculated from the estimated positions of registered femur and tibia per time-frame using the method describe by Grood and Suntay [29].

\subsubsection{Cadaver experiment}

A full-body specimen cadaver (male, $79 \mathrm{~kg}, 179 \mathrm{~cm}$ ) was obtained from and approved by the Anatomy Department of the Radboud medical center (RUMC), Nijmegen, the Netherlands. There was no history of illness, injury, or treatment affecting the knee or hip joint for this specimen. Prior to the experiment, the dimensions of several anatomical regions were measured, including the circumferences of the middle parts of thigh and shank, the distal femur, the proximal tibia and the ankle for designing subject-specific ultrasound holders. In addition, two intra-cortical bone pins with four optical markers were inserted to the femur and tibia separately (i.e. four intra-cortical bone pins and 16 optical marker in total) to track actual motion of the leg and to obtain gold standard kinematics (Figure 4.3-A). After inserting all intra-cortical bone pins, a CT scan was made at the Department of Radiology of the RUMC using a TOSHIBA Aquilion ONE (TOSHIBA, Tustin, USA) with a voxel size of $0.755 \mathrm{~mm} \times 0.755 \mathrm{~mm} \times 0.500 \mathrm{~mm}$. After the CT scan, the bone models of the femur and tibia were manually segmented from the CT images to generate a surface mesh of the bone in STL format using Mimics ${ }^{\circledR}$ 17.0. The 3D locations of the optical markers mounted on four intra-cortical bone pins were also digitized in the CT image coordinate system.

During the experiment, the upper body of the specimen was fixated by nylon straps on the surgical table and the flexion angle of right leg was manually move cyclically from flexion to extension to simulate swinging phase (Figure 4.4-a, b). The cycle started from flexion to extension and flexed back to starting point, in total seven cyclic motions. Six custom A-mode ultrasound holders were mounted on the low extremity (Figure 4.3-B). All optical markers (16 markers for bone pins, 18 markers for ultrasound holders) were concurrently tracked by the Visualeyez optical tracking system (VZ4000z) with three calibrated trackers operating at $60 \mathrm{~Hz}$ (Figure 4.3-C). The ultrasound signal was captured and synchronized with the Visualeyez tracking system in the Diagnostic Sonar FI Toolbox with 2.3GHZ CPU (Intel Core i7-3610QE) and 8GB RAM. The sample rate of acquisition was $25 \mathrm{~Hz}$. Custom written software (LabVIEW 2015) was developed to provide a graphical user interface for storing all 
raw data and for post-processing. The gold standard tibiofemoral kinematics and ultrasound derived tibiofemoral kinematics were quantified together to assess the accuracy of the multichannel 3D-tracked A-mode ultrasound tracking system. Mean, Standard deviation (SD) and Root-Mean-Square (RMS) errors of absolute differences between these two sets of kinematics were calculated. To illustrate the differences of two sets of kinematics, 6 DOF kinematics were plotted as a function of increasing sample number. Since the cadaveric leg was manipulated in a periodic fashion, the full lengths of two sets of kinematics were also cropped into seven cyclic motions which started from flexion to extension and back to flexion as a completed cyclic motion. The lengths of cropped cyclic motions were normalized in order to illustrate 6 DOF kinematics with the corresponding percentage of cyclic motion.

Table 4.1 Mean, Standard Deviation (SD) and Root-Mean-Square (RMS) errors of absolute differences between the ultrasound derived tibiofemoral kinematics and bone-pin kinematics (gold standard): Flexion-Extension (Flex/Ext), AdductionAbduction (Add/Abd), External-Internal (Ext/Int) rotations; Anterior-Posterior (Ant/Post), Proximal-Distal (Prox/Dist) and Lateral-Medial (Lat/Med) translations

\begin{tabular}{lllllll}
\hline \hline & \multicolumn{2}{c}{ Joint rotational errors $\left(^{\circ}\right)$} & & \multicolumn{3}{c}{ Joint translational errors (mm) } \\
\cline { 2 - 7 } & Flex/Ext & Add/Abd & Ext/Int & Ant/Post & Prox/Dist & Lat/Med \\
\hline Mean & 1.32 & 0.71 & 2.49 & 4.55 & 3.08 & 1.80 \\
SD & 0.73 & 0.52 & 2.14 & 2.17 & 1.57 & 1.41 \\
RMS & 1.51 & 0.88 & 3.28 & 5.04 & 3.45 & 2.29 \\
\hline \hline
\end{tabular}


A Novel Multi-channel 3D-Tracked A-Mode Ultrasound System to Measure Tibiofemoral Kinematics

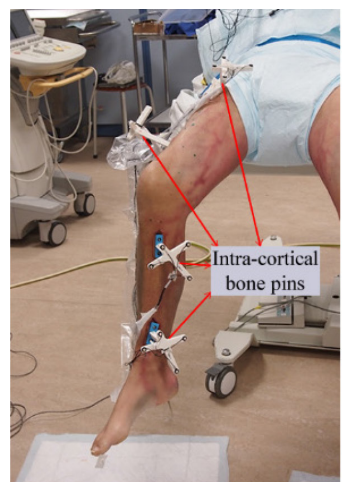

(a)

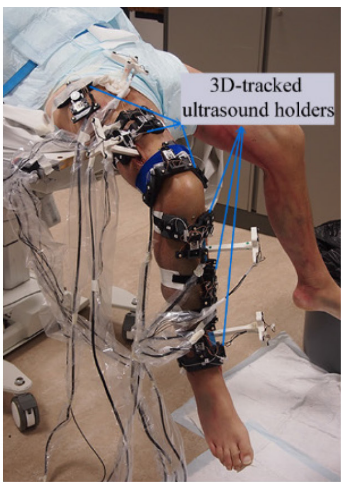

(b)

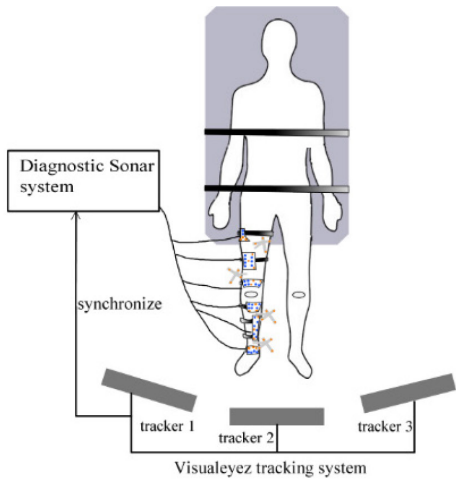

(c)

Figure 4.3 (a) Four intra-cortical bone pins was inserted into the low extremity to produce gold standard kinematics, two in the femur and two on the tibia; (b) Six 3D-tracked ultrasound holders attached on the lower extremity produced the ultrasound derived kinematics. (c) A schematic of a cadaver experiment showing the multi-channel A-mode ultrasound tracking system comprised of Visualeyez tracking system and Diagnostic Sonar system.

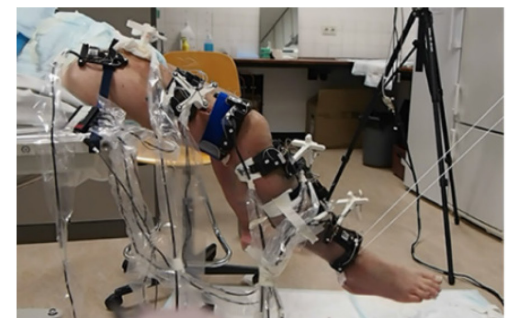

(a)

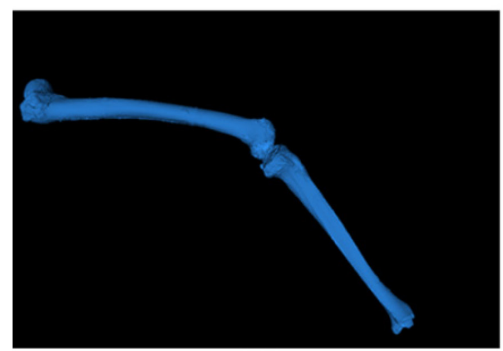

(c)

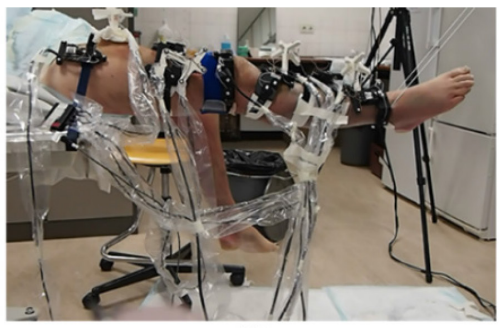

(b)

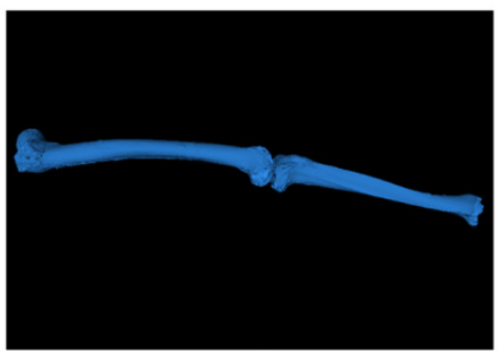

(d)

Figure 4.4 (a) the knee joint of specimen was kept at a certain flexion angle. (b) the knee joint of specimen was moved to full extension. (c) the estimated 3D positions of the registered femur and tibia at a corresponding flexion angle as (a). (d) the estimated 3D positons of the registered femur and tibia at same extension pose as (b).

\subsection{Results}

Flexion (+) / extension (-) rotation, adduction (+) / abduction (-) rotation and lateral (+) / medial (-) followed the trends of the gold standard kinematic components (Figure 4.5 and 


\section{A Novel Multi-channel 3D-Tracked A-Mode Ultrasound System to Measure}

Tibiofemoral Kinematics

4.6). For the Anterior (+) / Posterior (-) and Proximal (+) / distal (-) translations, there were systematic errors between the gold standard and ultrasound derived kinematics.

Root-Mean-Square (RMS) errors ranged from $0.88^{\circ}$ to $3.28^{\circ}$ for joint rotations and ranged from $2.29 \mathrm{~mm}$ to $5.04 \mathrm{~mm}$ for joint translations. The largest rotational error was associated with external-internal rotation whereas the anterior-posterior translation produced the largest translational error. Average rotational errors of $1.51 \pm 1.13^{\circ}$ (mean $\pm \mathrm{SD}$ ) and average translational errors of $3.14 \pm 1.72 \mathrm{~mm}$ (mean $\pm \mathrm{SD}$ ) were noted for ultrasound derived kinematics relative to gold standard.

\subsection{Discussion}

The purpose of this study was to describe a novel tibiofemoral kinematics measurement system based on multiple 3D-tracked A-mode ultrasound system, to prove the feasibility of this method and to assess its achievable accuracy by performing a cadaveric experiment. As we demonstrated, the multi-channel 3D-tracked A-mode ultrasound tracking system was capable of measuring 3D knee joint motion during simulated flexion-extension movements in a dynamic fashion. The novelty of this study is the combination of two existed techniques (3D motion capture and ultrasound technique) and to use their advantages for developing a new methodology to explore the possibility to track the motion of bone segments in the knee joint without invasiveness and lack of radiation as well as to provide a relatively high accuracy. We originally set the desired accuracy of measured kinematics by the multichannel 3D-tracked A-mode ultrasound system to be less than $1 \mathrm{~mm}$ and $1^{\circ}$ for translational and rotational errors, respectively. Thereby we could be close to the current state-of-art biplane fluoroscopic systems $[30,31]$ so that our system could serve as an alternative to measure prosthetic kinematics in TKA patients and gait analysis.

The smallest RMS error was $0.88^{\circ}$ for Abduction(+)/Adduction(-), which has achieved sub-degree accuracy and is comparable with fluoroscopic systems with $0.77^{\circ} \mathrm{RMS}$ error [14]. The maximum RMS errors were $3.28^{\circ}$ and $5.04 \mathrm{~mm}$ for external(+)/Internal(-) rotation and Anterior(+)/Posterior(-) translation. However, the largest translational error maybe caused by a systematic deviation in registration. Further study is necessary to tackle this erroneous registration outcome in order to meet the required accuracy for prosthetic kinematic measurement. 

Tibiofemoral Kinematics
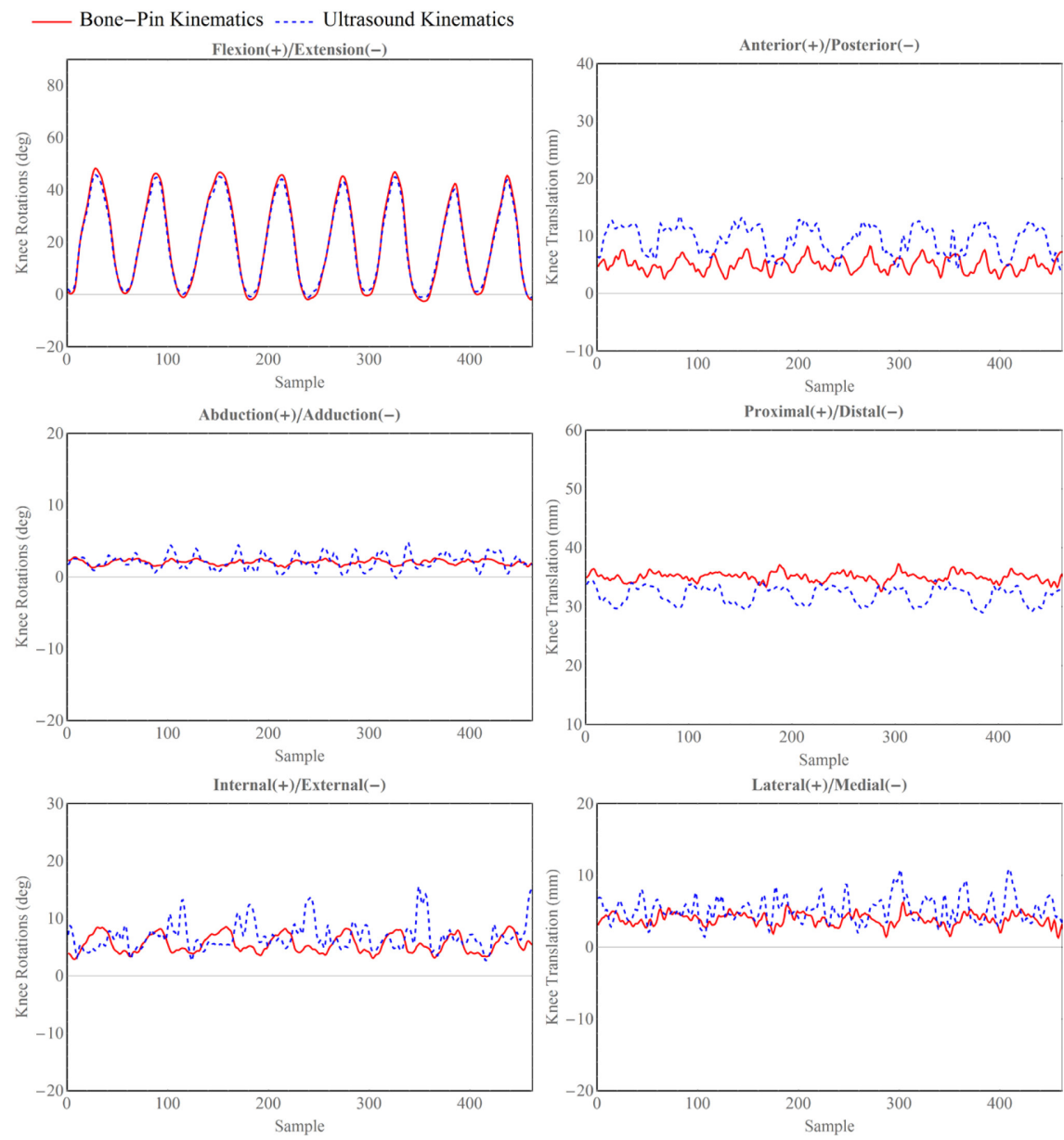

Figure 4.5 Comparisons of the ultrasound derived tibiofemoral kinematics (blue dashed lines) and gold standard kinematics derived from bone pins (red, solid line) on knee joint flexion(+)/extension(-), adduction(+)/abduction(-) and external(+)/internal(-) rotations and anterior $(+) /$ posterior(-), proximal $(+) / \operatorname{distal}(-)$ and lateral $(+) /$ medial(-) translations.

Compared to skin-mounted markers, multi-channel A-mode US system has the potential to provide more accurate tibiofemoral kinematics, since the bone position and orientation were estimated from the bony points instead of the positions of markers on the skin. Hence, it has the potential to overcome the effect of STA. In a skin-mounted marker system, STA are generally generated and their effects are difficult to eliminate $[4,5]$, because the inherent mismatch between skin and bone movement is difficult to remove under all circumstances. Andersen et al. reported that the inclusion of optimized knee joint constraints did not eliminate or reduce the effect of STA and improve the validity of reconstructed kinematics [32] for skin marker systems. The reported accuracy of skin-mounted marker system showed 


\section{A Novel Multi-channel 3D-Tracked A-Mode Ultrasound System to Measure Tibiofemoral Kinematics}

that average errors were up to $4.40^{\circ}$ and $13.0 \mathrm{~mm}$ for rotations and translations, respectively, for a walking motion task [3]. Our system the largest mean errors were up to $2.46^{\circ}$ and 4.55 $\mathrm{mm}$, which showed lower error than literature reported accuracy of skin markers. However, a more critical comparison is needed to evaluate the performance of our system against a skin-mounted marker system. When it comes to fluoroscopic systems, only Flexion/Extension and Abduction/Adduction rotations are relatively comparable with the accuracies of fluoroscopic system. The other motions had larger error than fluoroscopic systems. Therefore, the aim of further study is to improve the accuracy of the current system in order to get close to the desired accuracy and obtain comparable errors as obtained with fluoroscopic systems.
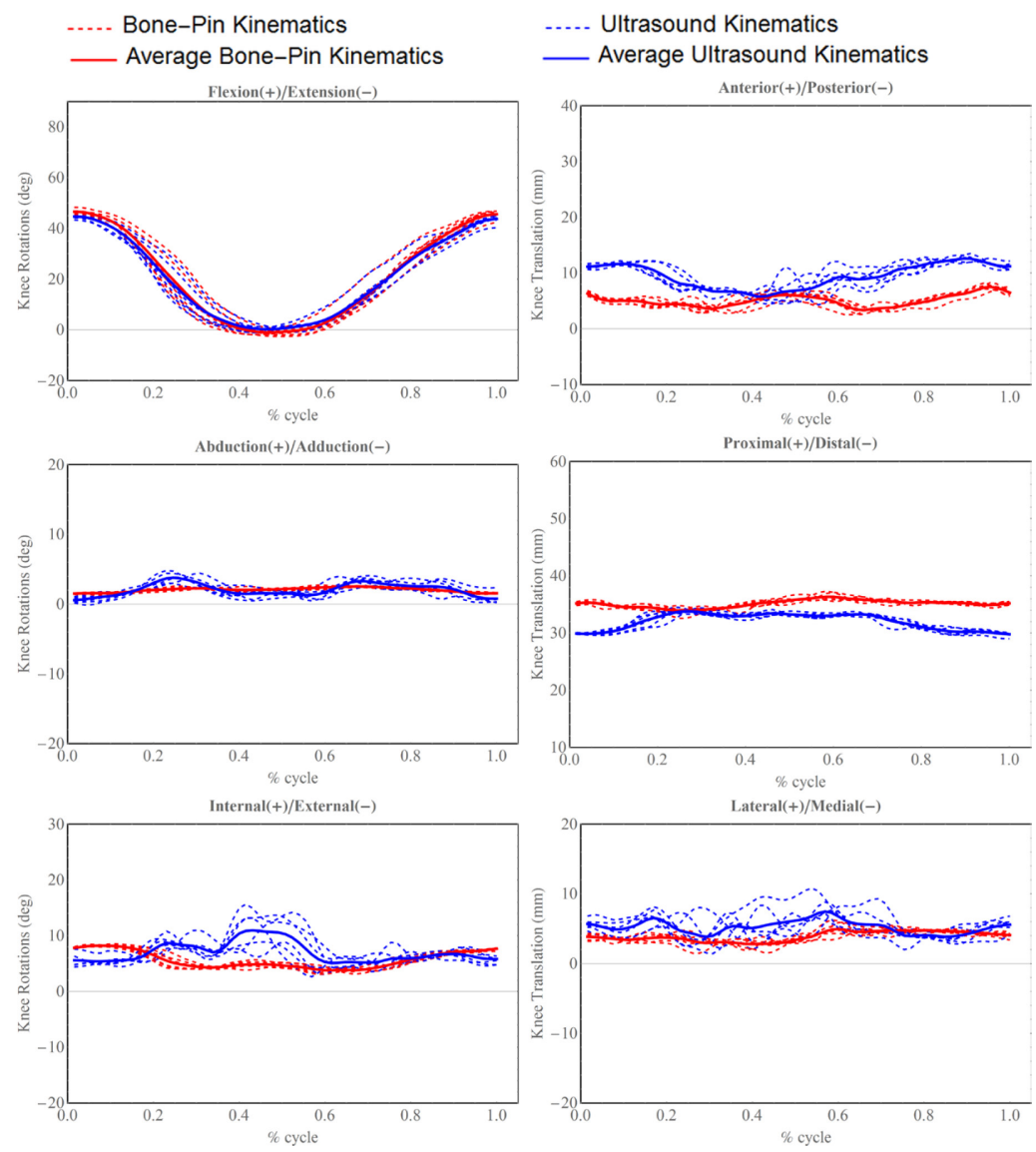

Figure 4.6 6 DOF tibiofemoral kinematics derived from ultrasound and bone pins are displayed in cyclical fashion. All cyclical trials of ultrasound derived kinematics are plotted in blue dash lines and the solid thick blue line represents average motion cycle estimated from ultrasound system; all bone pins trials are plotted in dashed red lines and solid thick red line represents the average motion cycle measured from bone pins. 


\section{A Novel Multi-channel 3D-Tracked A-Mode Ultrasound System to Measure Tibiofemoral Kinematics}

There are several factors contributing to the error of the ultrasound system reported in this study 1) loss of sight between ultrasound probe and bone during movement, i.e. during movement the bone can move out of the field of view of the ultrasound probe; 2) incorrect detection of the reflection peak, directly related to the distance between bone and ultrasound probe; 3) the inherent error of the registration algorithm; 4) the lack of geometric constraints in distal-proximal direction. The first and second factor are closely related, and strongly influenced by the incident angle of the A-mode ultrasound transducer [24, 33] affecting both the optimal field of view and the quality of the ultrasound echo-signal. By keeping the Amode ultrasound transducer perpendicular to the bone surface it will be easier to find the bone peak in the ultrasound echo-signal $[34,35]$. However, it is challenging to maintain the incident angle always perpendicular in practice, especially during movement. Also the specimen was lying on the surgical table and the soft tissues on the greater trochanter and proximal part of thigh were squeezed so that the depth of tissue-bone boundary increased in an abnormal fashion; this is obviously different from soft tissue deformation occurring during standing or walking circumstances. The bone to each transducer distance was determined from the ultrasound echo-signal. Sometimes the incorrect peak was selected and then untrue bone reflection points were inserted in the automatic registration algorithm. Hence, future work will focus on the improvement of keeping the sight of bone signal of A-mode US echo during movement and the development of more accurate and reliable peak detection methods. Factors 3) and 4) are interrelated. In our study, the number of A-mode US transducers was limited to 30 since it was just the first prototype. As the mid-shaft areas of femur and tibia are largely symmetric along the longitudinal axis, the errors along this axis are reasonably larger than around other axes. In the future, more A-mode ultrasound transducers will be added and relocated on newly designed custom US holders to cover more anatomical regions. More advanced registration algorithm will be adopted to improve registration accuracy in our scenario.

This study has several limitations. Firstly, we only used one specimen in the experiment. More specimens covering various sizes and BMIs could provide more valuable information regarding the 3D-tracked ultrasound holder design, improvement of maintaining good skin contact, ultrasound signal quality, and overall measurement robustness. Secondly, the specimen was placed on a surgical table and motions were generated manually. Muscles were obviously passively stretched and soft tissues may move differently as in living subject. However, to simulate the walking of a full body specimen is quite challenging to complete in a cadaveric session. Future studies will focus on the in-vivo validation.

Certainly, this study also has strengths. Firstly, intra-cortical bone pins provide gold standard kinematics, which were measured with all 3D-tracked ultrasound holders 


\section{A Novel Multi-channel 3D-Tracked A-Mode Ultrasound System to Measure}

Tibiofemoral Kinematics

concurrently. It avoided the synchronization problem when using different tracking modalities. Secondly, the customized 3D-tracked ultrasound holders were manufactured using an accurate 3D printing process, which simplified the procedure of calibrating all ultrasound holders. In addition, the working principle of 3D-tracked ultrasound holders facilitates the application of our system into clinical scenarios with simplification and reusage. Although our current system has a lower accuracy of reconstructed kinematics than most of fluoroscopic systems, it provides an approach to reconstruct tibiofemoral kinematics non-invasively and without any radiation. With the high potential to implement further improvements relative to the overall design of the system, the registration algorithms and peak detection methods, we expect to develop a system that the accuracy of the system to measure tibiofemoral kinematics will be comparable to that of fluoroscopic systems.

\subsection{Conclusion}

This study has presented a multi-channel A-mode ultrasound system and proven its feasibility of tracking tibiofemoral kinematics by means of a cadaver experiment. Although the reconstructed tibiofemoral kinematics were less accurate than those reported for fluoroscopic systems, it has the potential to overcome the effects of soft tissue artifacts of skin-mounted markers systems to produce more accurate kinematics. Thus, this multichannel A-mode ultrasound system provides a non-invasive and non-radiative method for measuring tibiofemoral kinematics, which could aid in clinical gait analysis and prosthetic kinematic measurement.

\section{Acknowledgment}

The authors also thank for the generous helps of Léon Driessen and Richard van Swam in cadaveric preparation from Orthopedic Research Lab, Radboud university medical center, Netherlands.

\section{References}

1. Fuller, J., et al., A comparison of lower-extremity skeletal kinematics measured using skin- and pin-mounted markers. Human Movement Science, 1997. 16(2-3): p. 219-242.

2. Lafortune, M.A., et al., Three-dimensional kinematics of the human knee during walking. Journal of Biomechanics, 1992. 25(4): p. 347-357.

3. Benoit, D.L., et al., Effect of skin movement artifact on knee kinematics during gait and cutting motions measured in vivo. Gait \& Posture, 2006. 24(2): p. 152-164. 
A Novel Multi-channel 3D-Tracked A-Mode Ultrasound System to Measure Tibiofemoral Kinematics

4. Cappozzo, A., et al., Position and orientation in space of bones during movement: anatomical frame definition and determination. Clin Biomech (Bristol, Avon), 1995. 10(4): p. 171-178.

5. Cappozzo, A., et al., Position and orientation in space of bones during movement: experimental artefacts. Clinical Biomechanics, 1996. 11(2): p. 90-100.

6. Cappozzo, A., et al., Surface-marker cluster design criteria for 3-D bone movement reconstruction. IEEE Transactions on Biomedical Engineering, 1997. 44(12): p. 1165-1174.

7. Ramsey, D.K. and P.F. Wretenberg, Biomechanics of the knee: methodological considerations in the in vivo kinematic analysis of the tibiofemoral and patellofemoral joint. Clinical Biomechanics, 1999. 14(9): p. 595-611.

8. Akbarshahi, M., et al., Non-invasive assessment of soft-tissue artifact and its effect on knee joint kinematics during functional activity. Journal of Biomechanics, 2010. 43(7): p. $4.1292-1301$.

9. Anderst, W., et al., Validation of Three-Dimensional Model-Based Tibio-Femoral Tracking During Running. Medical engineering \& physics, 2009. 31(1): p. 10-16.

10. Bingham, J. and G. Li, An Optimized Image Matching Method for Determining InVivo TKA Kinematics with a Dual-Orthogonal Fluoroscopic Imaging System. Journal of Biomechanical Engineering, 2006. 128(4): p. 588-595.

11. Giphart, J.E., et al., Accuracy of a contour-based biplane fluoroscopy technique for tracking knee joint kinematics of different speeds. Journal of Biomechanics, 2012. 45(16): p. 2935-2938.

12. Banks, S. and P. Flood, JOINTTRACK AUTO: AN OPEN-SOURCE PROGRAMME FOR AUTOMATIC MEASUREMENT OF 3D IMPLANT KINEMATICS FROM SINGLE- OR BI-PLANE RADIOGRAPHIC IMAGES. Bone \&amp; Joint Journal Orthopaedic Proceedings Supplement, 2016. 98-B(SUPP 1): p. 38-38.

13. Li, G., S.K. Van de Velde, and J.T. Bingham, Validation of a non-invasive fluoroscopic imaging technique for the measurement of dynamic knee joint motion. $\mathrm{J}$ Biomech, 2008. 41(7): p. 1616-22.

14. Guan, S., et al., Mobile Biplane X-Ray Imaging System for Measuring 3D Dynamic Joint Motion During Overground Gait. IEEE Trans Med Imaging, 2016. 35(1): p. 326-36.

15. Kozanek, M., et al., Tibiofemoral kinematics and condylar motion during the stance phase of gait. Journal of Biomechanics, 2009. 42(12): p. 1877-1884. 
16. Barratt, D.C., et al., Self-calibrating 3D-ultrasound-based bone registration for minimally invasive orthopedic surgery. IEEE Trans Med Imaging, 2006. 25(3): p. 312-23.

17. Amin, D.V., et al., Ultrasound registration of the bone surface for surgical navigation. Comput Aided Surg, 2003. 8(1): p. 1-16.

18. Otake, Y., et al., Intraoperative image-based multiview 2D/3D registration for image-guided orthopaedic surgery: incorporation of fiducial-based C-arm tracking and GPUacceleration. IEEE Trans Med Imaging, 2012. 31(4): p. 948-62.

19. Masum, M.A., et al., Accuracy assessment of Tri-plane B-mode ultrasound for noninvasive 3D kinematic analysis of knee joints. Biomed Eng Online, 2014. 13: p. 122.

20. Hamidzada, W.A. and E.P. Osuobeni, Agreement between A-mode and B-mode ultrasonography in the measurement of ocular distances. Vet Radiol Ultrasound, 1999. 40(5): p. $502-7$.

21. Horn, B.K.P., Closed-form solution of absolute orientation using unit quaternions. Journal of the Optical Society of America A, 1987. 4(4): p. 629-642.

22. Herring, J.L., et al., Surface-based registration of CT images to physical space for image-guided surgery of the spine: a sensitivity study. IEEE Trans Med Imaging, 1998. 17(5): p. $743-52$.

23. Ma, B. and R.E. Ellis, Robust registration for computer-integrated orthopedic surgery: laboratory validation and clinical experience. Med Image Anal, 2003. 7(3): p. 23750 .

24. Fieten, L., et al., Fast and accurate registration of cranial CT images with A-mode ultrasound. Int J Comput Assist Radiol Surg, 2009. 4(3): p. 225-37.

25. Besl, P.J. and H.D. McKay, A method for registration of 3-D shapes. IEEE Transactions on Pattern Analysis and Machine Intelligence, 1992. 14(2): p. 239-256.

26. Maurer, C.R., et al., Registration of 3-D images using weighted geometrical features. IEEE Trans Med Imaging, 1996. 15(6): p. 836-49.

27. Maurer, C.R., Jr., R.J. Maciunas, and J.M. Fitzpatrick, Registration of head CT images to physical space using a weighted combination of points and surfaces. IEEE Trans Med Imaging, 1998. 17(5): p. 753-61.

28. Miranda, D.L., et al., Automatic determination of anatomical coordinate systems for three-dimensional bone models of the isolated human knee. Journal of Biomechanics, 2010. 43(8): p. 1623-1626. 


\section{A Novel Multi-channel 3D-Tracked A-Mode Ultrasound System to Measure}

Tibiofemoral Kinematics

29. Grood, E.S. and W.J. Suntay, A joint coordinate system for the clinical description of three-dimensional motions: application to the knee. J Biomech Eng, 1983. 105(2): p. 13644.

30. Guan, S., et al., In vivo six-degree-of-freedom knee-joint kinematics in overground and treadmill walking following total knee arthroplasty. Journal of Orthopaedic Research, 2016: p. n/a-n/a.

31. Gray, H.A., S. Guan, and M.G. Pandy, Accuracy of mobile biplane X-ray imaging in measuring 6-degree-of-freedom patellofemoral kinematics during overground gait. Journal of Biomechanics, 2017. 57: p. 152-156.

32. Andersen, M.S., et al., Do kinematic models reduce the effects of soft tissue artefacts in skin marker-based motion analysis? An in vivo study of knee kinematics. Journal of Biomechanics, 2010. 43(2): p. 268-273.

33. Heger, S., et al., User-interactive registration of bone with A-mode ultrasound. IEEE Eng Med Biol Mag, 2005. 24(2): p. 85-95.

34. Chang, T.C., et al., A-Mode Ultrasound Bone Registration for Computer-Assisted Knee Surgery: Calibration and Robustness Test, in 25th Southern Biomedical Engineering Conference 2009, 15 - 17 May 2009, Miami, Florida, USA, A. McGoron, C.-Z. Li, and W.C. Lin, Editors. 2009, Springer Berlin Heidelberg. p. 97-100.

35. Mozes, A., et al., Three-dimensional A-mode ultrasound calibration and registration for robotic orthopaedic knee surgery. Int J Med Robot, 2010. 6(1): p. 91-101. 


\section{Chapter 5 In situ comparison of A-mode ultrasound tracking system and skin-mounted markers for measuring kinematics of the lower extremity}

Skin-mounted marker based motion capture systems are widely used in measuring the movement of human joints. Kinematic measurements associated with skin-mounted markers are subject to soft tissue artifacts (STA), since the markers follow skin movement, thus generating errors when used to represent motions of underlying bone segments. We present a novel ultrasound tracking system that is capable of compensating STA by means of measuring tibial and femoral bone surfaces during dynamic motions, and subsequently measuring six-degree-of-freedom (6-DOF) tibiofemoral kinematics. The aim of this study is to quantitatively compare the accuracy of tibiofemoral kinematics estimated by the ultrasound tracking system and by a conventional skin-mounted marker based motion capture system in a cadaveric experimental scenario. Two typical tibiofemoral joint models (spherical and hinge models) were used to derive relevant kinematic outcomes. Intra-cortical bone pins equipped with optical markers were inserted in the tibial and femoral bones to serve as a reference to provide ground truth kinematics. The ultrasound tracking system resulted in lower kinematic errors than the skin-mounted markers (the ultrasound tracking system: maximum root-mean-square (RMS) error $3.44^{\circ}$ for rotations and $4.88 \mathrm{~mm}$ for translations, skin-mounted markers with the spherical joint model: $6.32^{\circ}$ and $6.26 \mathrm{~mm}$, the hinge model: $6.38^{\circ}$ and $6.52 \mathrm{~mm}$ ). Our proposed ultrasound tracking system provides a potential for overcoming the effect of STA and mitigating its propagation to bone kinematics. Consequently, this technique could be considered as an alternative method for measuring 6DOF tibiofemoral kinematics, which may be adopted in gait analysis and clinical practice. 
In situ comparison of A-mode ultrasound tracking system and skin-mounted markers for measuring kinematics of the lower extremity

\subsection{Introduction}

Accurate measurements of joint kinematics are essential to comprehensively understand the mechanism of joint motion. Most of kinematic data on the lower extremity are obtained from motion capture systems, in which the trajectories of skin-mounted markers are recorded to represent the motion of underlying skeletal structure [1]. However, the estimated threedimensional (3D) position and orientation of bone segments and the related kinematics are subject to soft tissue artifacts (STA) [2]. Since the movement of the skin, muscles, and fat relative to the underlying bone is an inevitable phenomenon under dynamic motion tasks [3], the inherent mismatch between skin and bone movement is difficult to remove under all circumstances $[4,5]$. It has been reported that STA can cause measurement errors of up to 30 $\mathrm{mm}$ in the thigh [6]. The propagation of STA to knee joint kinematics has been reported to lead to average rotational errors of up to $4.4^{\circ}$ and $13.1^{\circ}$ and average translational errors of up to $13.0 \mathrm{~mm}$ and $16.1 \mathrm{~mm}$ for walking and cutting motions, respectively [7].

Extensive researches have been conducted on quantification, assessment, and compensation of STA for different motor tasks [8-13]. Multi-body kinematics optimization (MKO) has been used with the intent to compensate the STA and to limit the propagation of STA to joint kinematics estimation $[9,13]$. The typical mechanical linkages representing the knee joint and embedded in MKO are the hinge joint and the spherical joint, which involve major simplifications with respect to the actual joint and reduce the degrees of freedom (DOF) of the joint $[14,15]$. The hinge joint only allows the flexion-extension rotation [9]. The spherical joint allows all three rotations but no translation [16]. Researchers have realized that motion analysis research community should make more efforts in search of more advanced subject-specific joint models or error models, or a new measurement modality in order to improve the accuracy of estimated joint kinematics [17-19].

Instead of compensating STA from various perspectives of mathematical models and optimizations, we developed a new method to directly measure spatial information of underlying bones in order to produce an effective and valid representation of skeletal motion and the corresponding joint kinematics. A novel ultrasound (US) tracking system was developed to measure tibiofemoral kinematics dynamically, non-invasively, and without radiation. As A-mode ultrasound transducers are capable of detecting underlying bone surfaces non-invasively through multiple layers of soft tissues under dynamic movement, a combination of multiple A-mode ultrasound transducers and conventional skin-mounted markers provides a new approach of measuring the trajectories of multiple A-mode ultrasound transducers attached on the thigh and shank as well as detecting respective underlying bone surfaces from received ultrasound signals. Subsequently, the trajectories of 
In situ comparison of A-mode ultrasound tracking system and skin-mounted markers for measuring kinematics of the lower extremity

bone surfaces could be obtained and the relevant kinematics can be quantified. To evaluate our system relative to conventional motion capture systems with skin-mounted markers, cadaveric experiments were conducted.

The aim of the presented work is to compare tibiofemoral kinematics derived from our ultrasound tracking system with those kinematic outcomes derived from skin-mounted markers using two typical joint models (hinge and spherical) in cadaveric experiments. Another goal is to demonstrate the potential for overcoming the effect of STA and achieving high accuracy in estimated tibiofemoral kinematics with our ultrasound tracking system.

\subsection{Material and methods}

\subsubsection{Experimental setup}

A full body cadaveric specimen (male, $79 \mathrm{~kg}, 179 \mathrm{~cm}$ ) was obtained from and approved by the Anatomy Department of the Radboud University Medical Center (RUMC), Nijmegen, the Netherlands. There was no history of illness, injury, or treatment affecting the knee or hip functions. Tracked intra-cortical bone pins equipped with optical markers were inserted into the right femur (with two pins) and right tibia (with two pins), which provided ground truth kinematics as a reference. A CT scan was made at the Department of Radiology of the RUMC using a TOSHIBA Aquilion ONE (TOSHIBA, Tustin, USA) with a voxel size of $0.755 \mathrm{~mm}$ $\times 0.755 \mathrm{~mm} \times 0.500 \mathrm{~mm}$. Subsequently, the femur and tibia were manually segmented using Mimics 17.0 (Materialise N.V., Leuven, Belgium) to generate geometrical surface models of the bones, which were exported in STL format. The femoral and tibial anatomical reference frames were defined based on bone geometries using a method previously described [20]. The upper body of the cadaver was fixated with nylon straps in a supine position on a surgical table, while ensuring that the right leg could be manually displaced without any hindrances to its movement.

\subsubsection{Ultrasound tracking system}

The ultrasound tracking system comprised six customized 3D-tracked ultrasound holders covering six anatomical areas: greater trochanter of the femur, middle femoral shaft, lateral and medial sides of both femoral epicondyles and tibial condyles, middle tibial shaft, and ankle (Figure 5.1). Each ultrasound holder consisted of a variable number of A-mode ultrasound transducers (frequency: 7.5 MHz, Imasonic SAS, Voray / l'Ognon, France) and three optical markers that were tracked by Visualeyez VZ4000v system (PTI Phoenix Technologies Inc., Vancouver, Canada) operating at $60 \mathrm{~Hz}$. In total, $30 \mathrm{~A}$-mode ultrasound transducers and 18 optical markers were installed on six ultrasound holders. 
In situ comparison of A-mode ultrasound tracking system and skin-mounted markers for measuring kinematics of the lower extremity

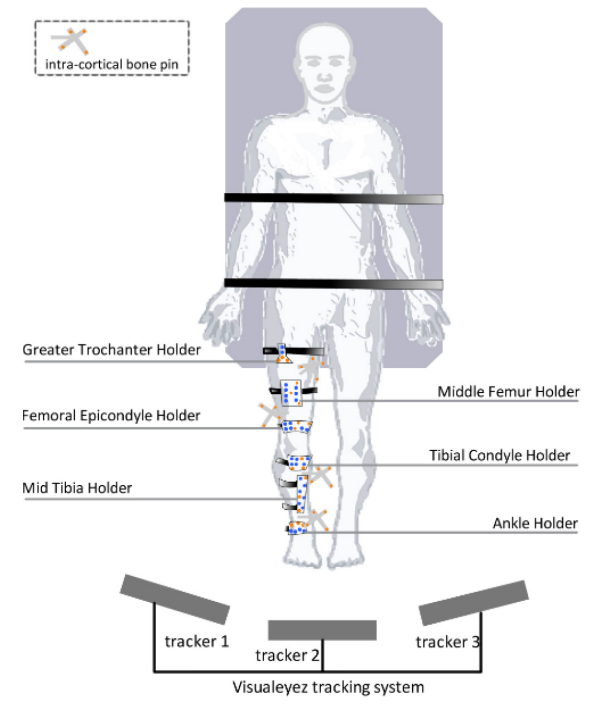

Figure 5.1 A schematic representation of measurement of ultrasound tracking system and the placement of six ultrasound holders. All inserted intra-cortical bone pins and attached six ultrasound holders were tracked and recorded concurrently by Visualeyez tracking system.

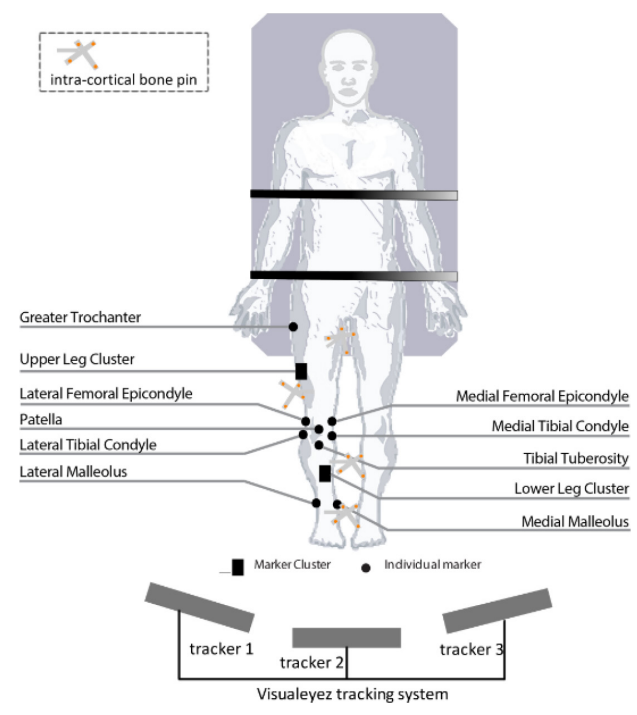

Figure 5.2 A schematic representation of skin-mounted marker measurement and configuration of marker placement. All intra-cortical bone pins and marker clusters and individual markers were tracked and recorded concurrently by Visualeyez tracking system.

After attaching all ultrasound holders on the lower extremity, the received ultrasound signals and trajectories of rigidly attached markers were collected to calculate corresponding points on the surface of the bones. Subsequently, these points were fed to a point-cloud registration algorithm [21] to estimate the position and orientation of the bone model in $3 \mathrm{D}$ 

markers for measuring kinematics of the lower extremity

space. Finally, tibiofemoral kinematics were quantified from the estimated bone positions per time-frame of acquisition using the established anatomical reference frames [22]. The relevant data acquisition and processing have been described previously in detail [21].The trajectories of inserted intra-cortical bone pins and attached ultrasound holders as well as received ultrasound signals were synchronized and recorded simultaneously.

\subsubsection{Skin-mounted markers measurement}

The skin-mounted marker measurement was performed by attaching active optical marker clusters and individual markers directly to the skin to track locations of anatomical landmarks which were measured by the same configuration of Visualeyez tracking system operating at $60 \mathrm{~Hz}$. The individual markers were placed directly on top of the skin of both the lateral and medial malleoli, tibial condyles, and femoral epicondyles, and of the greater trochanter, patella, and tibial tuberosity landmarks (Figure 5.2) [23]. Two marker clusters with three optical markers were firmly strapped on the middle part of the shank and thigh to reconstruct the used anatomical landmarks on the tibia and femur, respectively. Reconstruction of the related landmark positions was done by performing additional measurements using a fivemarker pointing device indicating landmark locations relative to the $3 \mathrm{D}$ positions of marker clusters, and the use of dedicated custom MATLAB scripts [24]. The positions of abovementioned anatomical landmarks were also measured by individual markers to ensure that reliable continuous data were available during dynamic movements, and to minimize soft-tissue artifacts within the choice of landmarks. The placement of skin-mounted markers is demonstrated in Figure 5.2. All the trajectories of attached marker clusters and individual markers accompanied with intra-cortical bone pins were measured and recorded simultaneously.

\subsubsection{Experimental trials}

Due to interferences in the placement of six ultrasound holders and skin-mounted markers altogether on the lower extremity, the cadaveric experiments needed to be performed in two independent experimental trials: 1) US-trial, i.e. ultrasound tracking system measurement with intra-cortical bone pins; 2) SKIN-trial, i.e. skin-mounted marker measurement with intra-cortical bone pins. During both trials, the same intra-cortical bone pins were used and recorded simultaneously with the other measurement technique. For each trial, the experiment consisted of a cyclic flexion and extension of the knee, performed manually. One motion cycle started from the initial pose (approximately $45^{\circ}$ of knee flexion), and proceeded to full-extension and ended back in the initial pose. The duration of one cycle was approximately 3 seconds. At least five successive motions cycles were recorded for each trial. 
In situ comparison of A-mode ultrasound tracking system and skin-mounted markers for measuring kinematics of the lower extremity

\subsubsection{Quantification of tibiofemoral kinematics}

All measurement data for US-trial were recorded in the Diagnostic Sonar FI Toolbox (Diagnostic Sonar Ltd, Livingston, UK) on a 2.3-GHz CPU (Intel Core i7-3610QE) computer equipped with 8 GB of RAM. Custom written software in LabVIEW 2015 (National Instruments, Austin, Texas, US) was developed to obtain 6-DOF tibiofemoral kinematics derived from ultrasound tracking system along with corresponding ground truth kinematics obtained from intra-cortical bone pins. The detailed description of post processing can be found in [21].

The SKIN-trial data were firstly processed in MATLAB R2015a (MathWorks, Natick, Massachusetts, US), by applying a bi-directional second-order low-pass Butterworth filter at a cut-off frequency of $20 \mathrm{~Hz}$ onto the marker coordinates and reconstructing landmarks following marker clusters. The identical bone models and established anatomical reference frames of the femur and tibia were imported into AnyBody Modeling System (AMS, version 6.0, AnyBody Technology, Aalborg, Denmark) as used in the US-trial. Anatomical landmark locations on the bone model were defined based on bone geometry. Individual markers were used for all indicated landmarks of Figure 5.2 except for the greater trochanter. The marker cluster attached to the middle thigh was used to reconstruct the pathway of the greater trochanter landmark, due to large soft-tissue artifacts within this position as a result of the cadaver setup.

Two models with different knee joint definitions were used to obtain kinematic outcomes; the hinge model and the spherical model, representing the two most widely used idealized joint definitions in kinematic analysis. Both models used the knee center of rotation as defined by the origin of the femoral anatomical reference frame [22]. However, the hinge model only allowed rotation in one degree of freedom, namely around the flexion-extension femoral axis, while the spherical model allowed all three rotations. Since only one kinematic DOF (flexion/extension) was allowed for the hinge model, the remaining translational and rotational kinematic components exhibited constant values. Specifically, the adduction/abduction and internal/external rotation remained at zero, whereas the translations assumed non-zero constant values, because of the tibial anatomical refrence frame being at a different location than the center of the knee joint (femoral anatomical frame). For the spherical model, the same consideraion for the translatons can be made as for the hinge model. Over-determinate kinematic analyses were carried out based on input spatial trajectories of processed landmarks [9], while enforcing the relevant kinematic constraints for each of the two knee joint definitions. The corresponding ground truth kinematics (i.e. obtained from the intra-cortical bone pins) were quantified using the same routine as for the US-trial [21]. 

markers for measuring kinematics of the lower extremity

US-trial
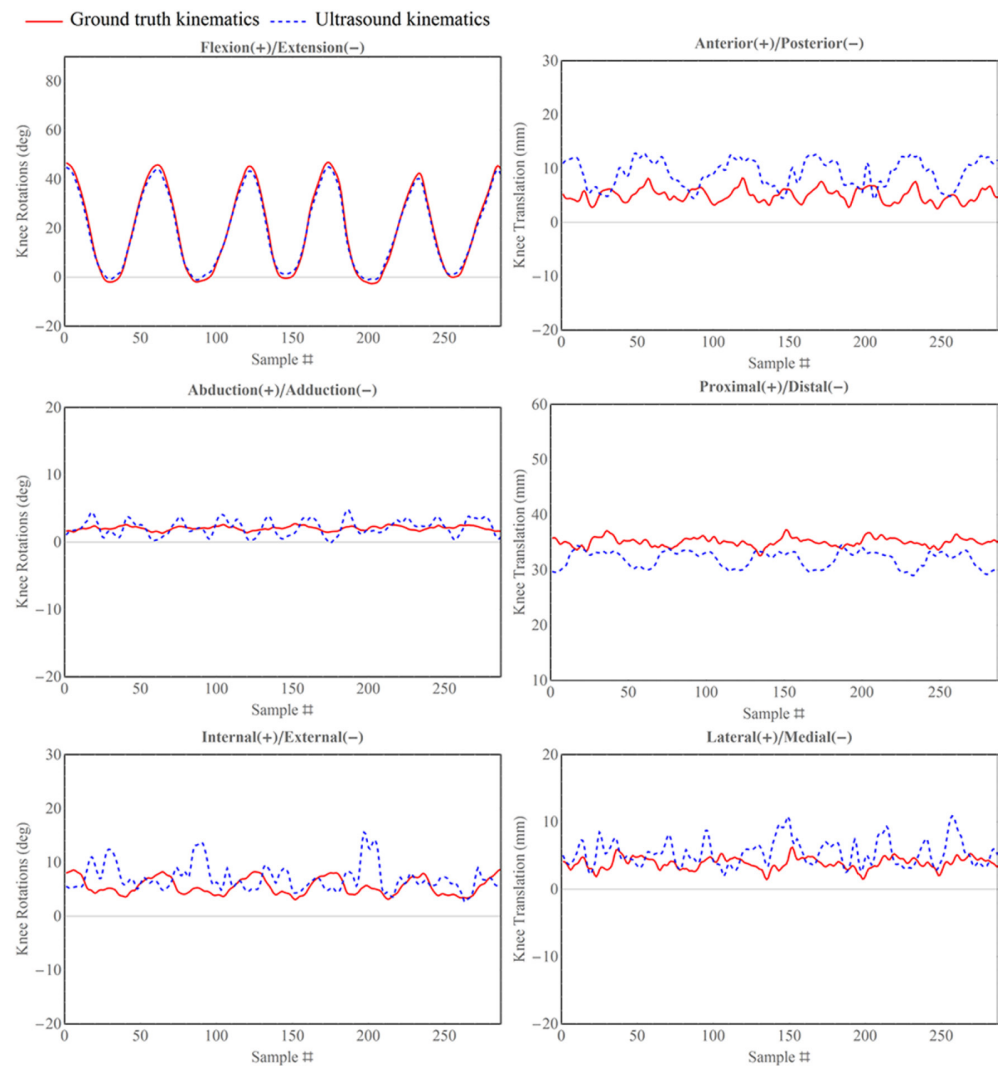

Figure 5.3 6-DOF tibiofemoral kinematic plots of US-trial in flexion-extension, abductionadduction, internal-external, anterior-posterior, proximal-distal, and lateral-medial kinematic components. Red solid line represents ground truth kinematics (reference) obtained from inserted intra-cortical bone pins; blue dashed line represents the kinematics derived from the ultrasound tracking system.

\subsubsection{Data analysis}

Absolute errors between the ground truth kinematics and the corresponding kinematics from the US-trial and SKIN-trial were calculated. Mean, standard deviation (SD), and rootmean-squared (RMS) errors were calculated. 6-DOF tibiofemoral kinematics were plotted as a function of increasing sample number for US-trial and SKIN-trial in Figure 5.3 and 5.4, respectively. In addition, the bias of estimated kinematics using the ultrasound tracking system, the spherical model, and the hinge model with respect to their respective ground truth kinematics were computed as a function of percentage of the motion cycle (Figure 5.5). The overall absolute errors for six kinematic components were illustrated in box whisker plots to 
In situ comparison of A-mode ultrasound tracking system and skin-mounted markers for measuring kinematics of the lower extremity

intuitively display the variety of estimated kinematic errors for the different methods (Figure 5.6).

Table 5.1 Mean \pm standard deviation (SD) of absolute errors describing the errors associated with three types of kinematic measurements (the ultrasound tracking system, the spherical joint model, and the hinge joint model).

\begin{tabular}{llll}
\hline \hline Mean \pm SD & $\begin{array}{c}\text { Ultrasound } \\
\text { tracking system }\end{array}$ & Spherical Joint & Hinge Joint \\
\hline Flex/Ext $\left(^{\circ}\right)$ & $1.39 \pm 0.66$ & $3.62 \pm 1.35$ & $3.20 \pm 1.24$ \\
Abd/Add $\left(^{\circ}\right)$ & $0.85 \pm 0.53$ & $3.23 \pm 1.01$ & $2.17 \pm 0.44$ \\
Int/Ext $\left(^{\circ}\right)$ & $2.65 \pm 2.20$ & $6.25 \pm 0.98$ & $6.12 \pm 1.79$ \\
Ant/Post $(\mathrm{mm})$ & $4.35 \pm 2.21$ & $5.82 \pm 2.31$ & $6.06 \pm 2.29$ \\
Prox/Dist $(\mathrm{mm})$ & $3.14 \pm 1.61$ & $4.65 \pm 2.34$ & $4.56 \pm 2.38$ \\
Lat/Med $(\mathrm{mm})$ & $2.00 \pm 1.53$ & $4.82 \pm 1.48$ & $6.39 \pm 1.25$ \\
\hline \hline
\end{tabular}


In situ comparison of A-mode ultrasound tracking system and skin-mounted markers for measuring kinematics of the lower extremity

SKIN-trial
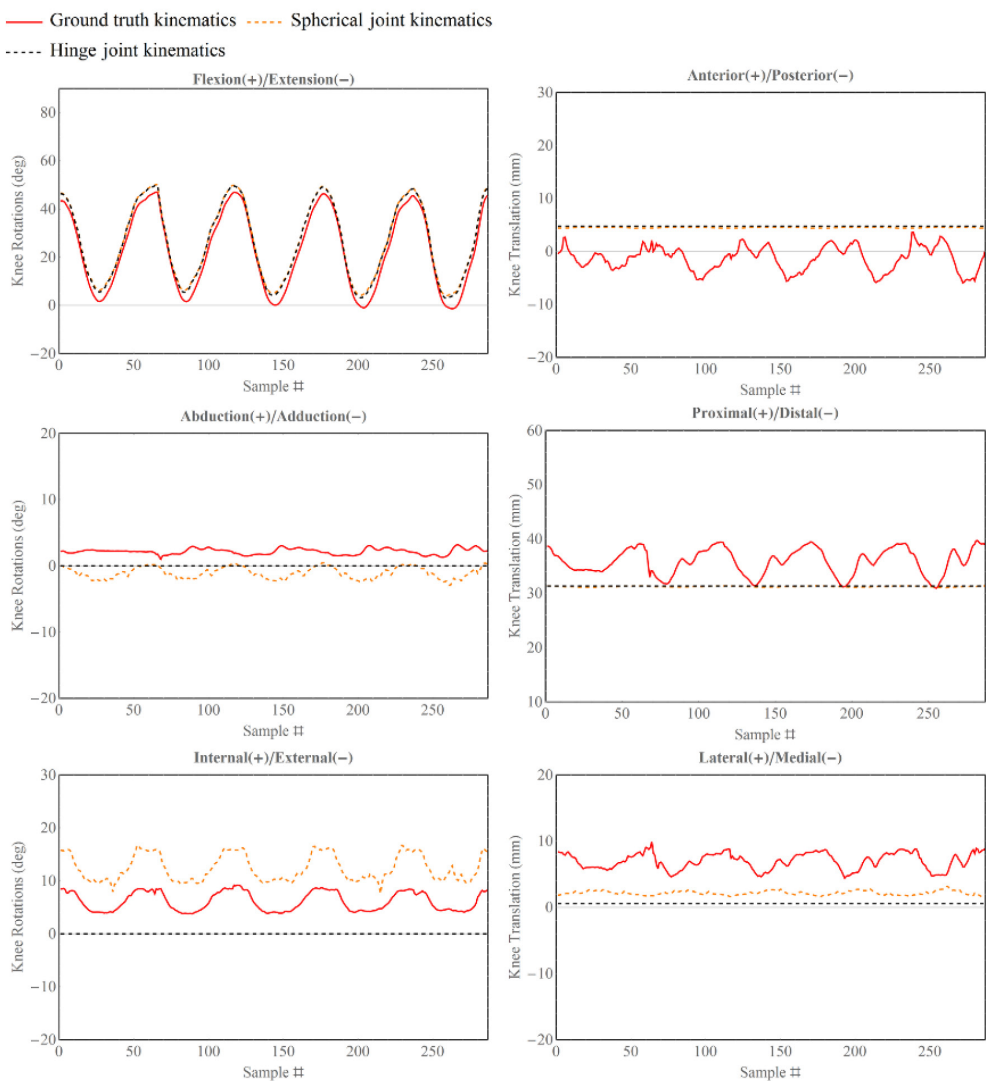

Figure 5.4 6-DOF tibiofemoral kinematic plots of SKIN-trial in flexion-extension, abductionadduction, internal-external, anterior-posterior, proximal-distal, and lateral-medial kinematic components. Red solid line represents ground truth kinematics (reference) obtained from inserted intra-cortical bone pins; orange dashed line represents the kinematics derived from the spherical joint model; black dashed line represents the kinematics derived from the hinge joint model. 
In situ comparison of A-mode ultrasound tracking system and skin-mounted markers for measuring kinematics of the lower extremity
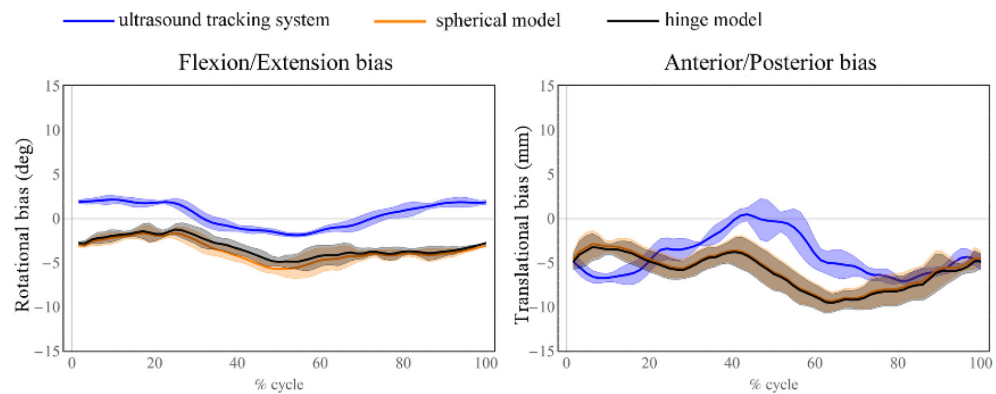

Abduction/Adduction bias
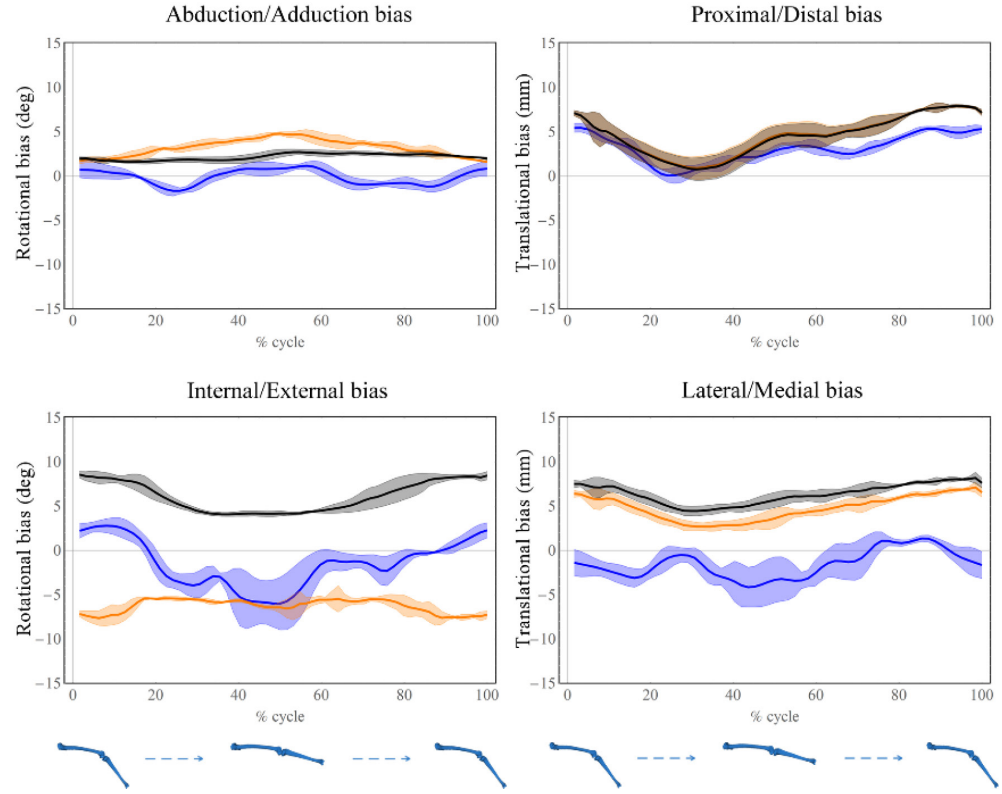

Figure 5.5 The biases of three kinds of kinematics (derived from the ultrasound tracking system, the spherical model, and the hinge model) with respect to ground truth kinematics were illustrated in 6-DOF kinematic components. The shaded curves represent mean \pm standard deviation of respective bias as a function of the percentage of the motion cycle. The biases of the ultrasound tracking system were indicated in blue. The biases of the hinge model and spherical model were indicated in black and in orange respectively.

\subsection{Results}

The mean rotational errors ranged from $3.23^{\circ}$ to $6.25^{\circ}$ and from $2.17^{\circ}$ to $6.12^{\circ}$ for the spherical model and hinge model, respectively. The mean translational errors ranged from 4.65 to $5.82 \mathrm{~mm}$ and from 4.56 to $6.39 \mathrm{~mm}$ for the spherical model and hinge model, respectively. For the ultrasound tracking system, the mean rotational errors $\left(0.85^{\circ}\right.$ to $\left.2.65^{\circ}\right)$ and mean translational errors ( 2.00 to $4.35 \mathrm{~mm}$ ) were lower than those errors of the two types of kinemaitc measurements (obtianted from the spherical and hinge models, Table 5.1). 
In situ comparison of A-mode ultrasound tracking system and skin-mounted markers for measuring kinematics of the lower extremity

Similarly lower errors were found in RMS errors for the ultrasound tracking system $\left(1.00^{\circ}\right.$ to $3.44^{\circ}$ and 2.52 to $\left.4.88 \mathrm{~mm}\right)$ compared with the spherical model $\left(3.38^{\circ}\right.$ to $6.32^{\circ}$ and 5.04 to $6.26 \mathrm{~mm})$ and the hinge model $\left(2.22^{\circ}\right.$ to $6.38^{\circ}$ and 5.14 to $6.52 \mathrm{~mm}$ ) (Table 5.2).
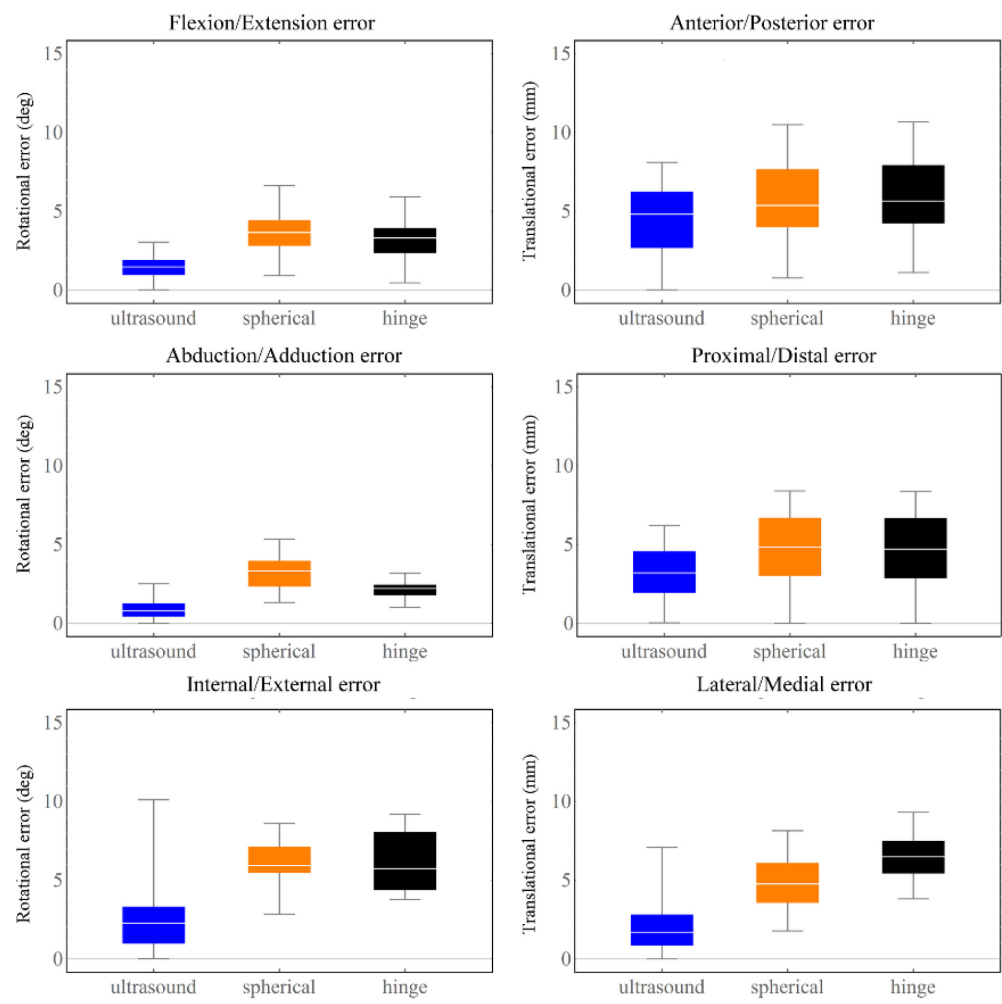

Figure 5.6 Box Whisker plots of absolute kinematic errors of three kinds of kinematics derived from the ultrasound tracking system (blue), the spherical model (orange), and the hinge model (black) with respect to the ground truth in six kinematic components.

\subsection{Discussion}

In this study, we performed cadaveric experiments in order to compare the measurement of our novel ultrasound tracking system with skin-mounted marker measurement for assessing the accuracy in estimated tibiofemoral kinematics in a highly controlled experimental scenario. Because of limited space on the thigh and shank, it was not possible to simultaneously attach all ultrasound holders and skin-mounted markers. Considering this challenge, intra-cortical bone pins were used as a reference for both separate measurements, which makes comparison feasible in this study. As the results have shown, the ultrasound tracking system could achieve relatively high accuracies in flexion/extension $\left(1.54^{\circ} \mathrm{RMS}\right.$ error) and abduction/adduction ( $1.00^{\circ} \mathrm{RMS}$ error), which is close to the accuracy of the 
In situ comparison of A-mode ultrasound tracking system and skin-mounted markers for measuring kinematics of the lower extremity

mobile fluoroscopic system with $0.77^{\circ}$ RMS error [25]. In addition, the ultrasound tracking system showed the potential to measure all 6-DOF kinematics with comparable accuracy by effectively reducing the propagation of STA to kinematic outcomes. In this study, the lower

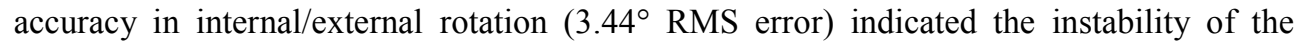
ultrasound tracking system when the leg was at full-extension pose (Figure 5.5, Internal/External bias). We believed this mainly originates from the lack of skin contact for several ultrasound holders caused by the deformations of surrounding soft tissue when stretching. Further research will focus on the improvement of the design of the ultrasound holders, especially regarding to skin contact.

Table 5.2 Root-mean-square (RMS) error describing the errors associated with three types of kinematic measurements (ultrasound tracking system, spherical joint model, and hinge joint model).

\begin{tabular}{llll}
\hline \hline RMS error & $\begin{array}{l}\text { Ultrasound } \\
\text { tracking system }\end{array}$ & Spherical Joint & Hinge Joint \\
\hline Flex/Ext $\left(^{\circ}\right)$ & 1.54 & 3.86 & 3.43 \\
Abd/Add $\left(^{\circ}\right)$ & 1.00 & 3.38 & 2.22 \\
Int/Ext $\left({ }^{\circ}\right)$ & 3.44 & 6.32 & 6.38 \\
Ant/Post $(\mathrm{mm})$ & 4.88 & 6.26 & 6.47 \\
Prox/Dist $(\mathrm{mm})$ & 3.53 & 5.20 & 5.14 \\
Lat/Med $(\mathrm{mm})$ & 2.52 & 5.04 & 6.52 \\
\hline \hline
\end{tabular}

Different to conventional motion capture systems using skin-mounted markers, we introduced an ultrasound technique into kinematic measurement, thus taking advantage of the capability of ultrasound transducer to measure underlying skeletal structure through soft tissues, which is an extension of the navigated A-mode ultrasound probes utilized in computer assisted orthopedic surgery [26-29]. In this study, an A-mode ultrasound transducer (single element ultrasound transducer) could be deemed as a virtually extended measurement line from the skin to the bone surface. Some researchers have proposed a similar concept by combining B-mode ultrasound transducer with a motion capture system, which could obtain one or multiple curves representing bone surfaces [30,31]. The potential of compensating STA has been validated in an in-vivo experiment by merely measuring the depth of bone surfaces on the greater trochanter with up to $16 \mathrm{~mm}$ measurement difference to skin marker 

markers for measuring kinematics of the lower extremity

measurement [30]. However, to our knowledge, dynamic tibiofemoral kinematic measurements have not been performed or validated in previous studies.

The framework for processing measured datasets of skin-mounted markers has been developed by many researchers, containing different kinds of MKO models besides the hinge and spherical models which were used for comparison in this study, e.g. parallel [12], coupling [10], and elastic [32] models. The placement and configuration of skin-mounted markers have also been investigated to assess the STA measurement error [33]. Although those findings showed a certain extent of mitigation of measurement errors related to STA, the validity of representing actual position and orientation of bone segments and related joint kinematics have not been improved $[18,19]$. This difficulty stems from the principle that processing skin-mounted markers cannot provide straightforward measurement of the underlying bone [34]. Current models and mathematical optimizations are unable to tackle this difficulty completely. Only invasive or fluoroscopic methods are currently capable of assessing joint kinematics with desirable level of accuracy of $\left(2^{\circ}\right.$ and $\left.2 \mathrm{~mm}\right)$ [19]. Based on the current study, the ultrasound tracking system showed the potential of being considered as an alternative to measure joint kinematics in the near future. It provides a new approach of compensating STA by actually measuring bone surfaces. Thus, this approach also has the potential to mitigate the effect of STA and to reduce the propagation of STA to final kinematics. In addition, no matter how the ultrasound holder shifts with respect to the underlying bone (i.e. either ultrasound holder to skin or skin to bone movements), as long as the underlying bone segment remains within the "field of view" of ultrasound transducer, valid bony points can be captured and the registration algorithm could find an appropriate transformation to match the bone model to the point cloud of bony points and quantify 6DOF tibiofemoral kinematics. When using skin-mounted markers, this type of shifting would contaminate the quality of skin-mounted marker dataset.

This work has several limitations. First, only one cadaveric specimen was used for the experiments. Ideally, a cohort of living subjects covering different sizes and BMIs and using advanced biplane fluoroscopic systems as the ground truth measurement could provide more valuable information regarding to STA and its effects on kinematics, which is related to an in-vivo validation study of the ultrasound tracking system for future work. Second, the ultrasound tracking system and skin-mounted marker measurement were used consecutively instead of simultaneously. However, using two independent measurement trials accompanied with the same intra-cortical bone pins serving as ground truth makes the quantitative comparison feasible and justifiable in this study. Third, only hinge and spherical models were used for comparison, which have their own limited DOF in final kinematic outcomes. However, these two models are the most widely used representations of the knee joint in 
In situ comparison of A-mode ultrasound tracking system and skin-mounted markers for measuring kinematics of the lower extremity

motion analysis. Fourth, knee motion of cadaveric leg was manipulated manually and the posture of body differed from the normal posture, e.g. walking. Subsequently, the muscle and soft tissue were passively stretched and compressed, which also differed from those during gait. However, to reproduce the locomotion of normal gait is quite challenging in a cadaveric setting. Further studies will move towards the assessment and validation of the ultrasound tracking system on the living subjects performing daily activities. To realize this goal, some improvements will be completed first, such as the redesign of the ultrasound holders for better skin contact and the employment of an advanced registration algorithm. With the high potential to implement further improvements associated with the ultrasound tracking system, we expect that the ultrasound tracking system could estimate 6-DOF tibiofemoral kinematics with an accuracy within the range of fluoroscopy measurements [25, 35] and provide a valid representation of bone segments in the knee joint, which will aid in gait analysis and disclosure of some pathological features.

\subsection{Conclusion}

In the present study we proposed a quantitative comparison of the estimations of tibiofemoral kinematics using the ultrasound tracking system versus skin-mounted markers with hinge and spherical models. The results showed that the ultrasound tracking method provides a valuable alternative to compensate STA and to improve the accuracy of kinematics estimation. This new ultrasound based kinematic measurement technique thus provides a useful approach to study joint motion for musculoskeletal modeling and biomechanical research communities.

\section{Acknowledgements}

The research leading to these results has received funding from the European Research Council under the European Union's Seventh Framework Programme (FP/2007-2013)/ERC Grant Agreement n.323091 awarded to N. Verdonschot. The authors thank for the generous helps of Léon Driessen and Richard van Swam in the cadaveric preparation from Orthopedic Research Lab, Radboud University Medical Center, Netherlands. The authors also thank for the technical help of Mats Boeve in the design of ultrasound holders from University of Twente, Netherlands.

\section{References}

1. Lafortune, M.A., et al., Three-dimensional kinematics of the human knee during walking. Journal of Biomechanics, 1992. 25(4): p. 347-357. 
In situ comparison of A-mode ultrasound tracking system and skin-mounted markers for measuring kinematics of the lower extremity

2. Cereatti, A., et al., Standardization proposal of soft tissue artefact description for data sharing in human motion measurements. J Biomech, 2017.

3. Dumas, R. and E. Jacquelin, Stiffness of a wobbling mass models analysed by a smooth orthogonal decomposition of the skin movement relative to the underlying bone. $\mathrm{J}$ Biomech, 2017.

4. Cappozzo, A., et al., Position and orientation in space of bones during movement: anatomical frame definition and determination. Clin Biomech (Bristol, Avon), 1995. 10(4): p. 171-178.

5. Cappozzo, A., et al., Position and orientation in space of bones during movement: experimental artefacts. Clinical Biomechanics, 1996. 11(2): p. 90-100.

6. Akbarshahi, M., et al., Non-invasive assessment of soft-tissue artifact and its effect on knee joint kinematics during functional activity. Journal of Biomechanics, 2010. 43(7): p. 1292-1301.

7. Benoit, D.L., et al., Effect of skin movement artifact on knee kinematics during gait and cutting motions measured in vivo. Gait \& Posture, 2006. 24(2): p. 152-164.

8. Cappozzo, A., et al., Surface-marker cluster design criteria for 3-D bone movement reconstruction. IEEE Transactions on Biomedical Engineering, 1997. 44(12): p. 1165-1174. 9. Andersen, M.S., M. Damsgaard, and J. Rasmussen, Kinematic analysis of overdeterminate biomechanical systems. Comput Methods Biomech Biomed Engin, 2009. 12(4): p. 371-84.

10. Bonnechère, B., et al., Physiologically corrected coupled motion during gait analysis using a model-based approach. Gait \& Posture, 2015. 41(1): p. 319-322.

11. Charlton, I.W., et al., Repeatability of an optimised lower body model. Gait \& Posture, 2004. 20(2): p. 213-221.

12. Duprey, S., L. Cheze, and R. Dumas, Influence of joint constraints on lower limb kinematics estimation from skin markers using global optimization. Journal of Biomechanics, 2010. 43(14): p. 2858-2862.

13. Lu, T.W. and J.J. O'Connor, Bone position estimation from skin marker coordinates using global optimisation with joint constraints. Journal of Biomechanics, 1999. 32(2): p. 129-134.

14. Reinbolt, J.A., et al., Determination of patient-specific multi-joint kinematic models through two-level optimization. J Biomech, 2005. 38(3): p. 621-6.

15. Ojeda, J., J. Martinez-Reina, and J. Mayo, A method to evaluate human skeletal models using marker residuals and global optimization. Mechanism and Machine Theory, 2014. 73: p. 259-272. 
In situ comparison of A-mode ultrasound tracking system and skin-mounted markers for measuring kinematics of the lower extremity

16. Clement, J., et al., Can generic knee joint models improve the measurement of osteoarthritic knee kinematics during squatting activity? Comput Methods Biomech Biomed Engin, 2017. 20(1): p. 94-103.

17. Cereatti, A., U. Della Croce, and A. Cappozzo, Reconstruction of skeletal movement using skin markers: comparative assessment of bone pose estimators. Journal of NeuroEngineering and Rehabilitation, 2006. 3: p. 7-7.

18. Andersen, M.S., et al., Do kinematic models reduce the effects of soft tissue artefacts in skin marker-based motion analysis? An in vivo study of knee kinematics. Journal of Biomechanics, 2010. 43(2): p. 268-273.

19. Richard, V., A. Cappozzo, and R. Dumas, Comparative assessment of knee joint models used in multi-body kinematics optimisation for soft tissue artefact compensation. $\mathrm{J}$ Biomech, 2017.

20. Miranda, D.L., et al., Automatic determination of anatomical coordinate systems for three-dimensional bone models of the isolated human knee. Journal of Biomechanics, 2010. 43(8): p. 1623-1626.

21. Niu, K., et al. A Novel Tibiafemoral Kinematics Measurement System Based on Multi-Channel A-Mode Ultrasound System. in CAOS 2017. 17th Annual Meeting of the International Society for Computer Assisted Orthopaedic Surgery. 2017. Aachen, Germany: EasyChair.

22. Grood, E.S. and W.J. Suntay, A joint coordinate system for the clinical description of three-dimensional motions: application to the knee. J Biomech Eng, 1983. 105(2): p. 13644.

23. Dumas, R., L. Cheze, and J.P. Verriest, Adjustments to McConville et al. and Young et al. body segment inertial parameters. J Biomech, 2007. 40(3): p. 543-53.

24. Soderkvist, I. and P.A. Wedin, Determining the movements of the skeleton using well-configured markers. J Biomech, 1993. 26(12): p. 1473-7.

25. Guan, S., et al., Mobile Biplane X-Ray Imaging System for Measuring 3D Dynamic Joint Motion During Overground Gait. IEEE Trans Med Imaging, 2016. 35(1): p. 326-36.

26. Chang, T.C., et al., A-Mode Ultrasound Bone Registration for Computer-Assisted Knee Surgery: Calibration and Robustness Test, in 25th Southern Biomedical Engineering Conference 2009, 15 - 17 May 2009, Miami, Florida, USA, A. McGoron, C.-Z. Li, and W.C. Lin, Editors. 2009, Springer Berlin Heidelberg. p. 97-100.

27. Amstutz, C., et al., A-mode ultrasound-based registration in computer-aided surgery of the skull. Arch Otolaryngol Head Neck Surg, 2003. 129(12): p. 1310-6.

28. Heger, S., et al., [Visual user guidance for registration based on A-mode ultrasound]. Biomed Tech (Berl), 2002. 47 Suppl 1 Pt 1: p. 61-4. 
In situ comparison of A-mode ultrasound tracking system and skin-mounted markers for measuring kinematics of the lower extremity

29. Fieten, L., et al., Fast and accurate registration of cranial CT images with A-mode ultrasound. Int J Comput Assist Radiol Surg, 2009. 4(3): p. 225-37.

30. Jia, R., et al., CAT \& MAUS: A novel system for true dynamic motion measurement of underlying bony structures with compensation for soft tissue movement. Journal of Biomechanics, 2017.

31. Masum, M.A., et al., Accuracy assessment of Tri-plane B-mode ultrasound for noninvasive 3D kinematic analysis of knee joints. Biomed Eng Online, 2014. 13: p. 122.

32. Richard, V., et al., Knee Kinematics Estimation Using Multi-Body Optimisation Embedding a Knee Joint Stiffness Matrix: A Feasibility Study. PLoS One, 2016. 11(6): p. $\mathrm{e} 0157010$.

33. Garling, E.H., et al., Soft-tissue artefact assessment during step-up using fluoroscopy and skin-mounted markers. J Biomech, 2007. 40 Suppl 1: p. S18-24.

34. Leardini, A., et al., Human movement analysis using stereophotogrammetry: Part 3. Soft tissue artifact assessment and compensation. Gait \& Posture, 2005. 21(2): p. 212-225. 35. Guan, S., et al., In vivo six-degree-of-freedom knee-joint kinematics in overground and treadmill walking following total knee arthroplasty. Journal of Orthopaedic Research, 2016: p. n/a-n/a. 

A novel ultrasound tracking system to track in-vivo knee joint motions during walking and stair descent: a feasibility study

\section{Chapter 6 A novel ultrasound tracking system to track in-vivo knee joint motions during walking and stair descent: a feasibility study}

Tracking joint motion of the lower extremity is important for human motion analysis. In this study, we present a novel ultrasound-based motion tracking system for measuring threedimensional (3D) position and orientation of the femur and tibia in 3D space and quantifying tibiofemoral kinematics under dynamic conditions. As ultrasound is capable of detecting underlying bone surface non-invasively through multiple layers of soft tissues, an integration of multiple A-mode ultrasound transducers with a conventional motion tracking system provides a new approach to track the motion of bone segments during dynamic conditions. To demonstrate the technical and clinical feasibility of this concept, an in-vivo experiment was conducted. For this purpose the variations of soft tissue thickness as well as the kinematics of healthy individuals were determined in treadmill walking conditions and stair descending tasks.

The results showed to be able to quantify various levels of soft tissue thickness change, depending on the location of the measurement. Furthermore, we clearly demonstrated the potential of tracking skeletal motion of the lower extremity and measuring 6 degree-offreedom tibiofemoral kinematics and related kinematic alterations caused by a variety of gait parameters. It was concluded that this prototyping system has great potential to measure human kinematics in an ambulant, non-radiative and non-invasive manner. 
A novel ultrasound tracking system to track in-vivo knee joint motions during walking and stair descent: a feasibility study

\subsection{Introduction}

Measuring skeletal motion occurring in the human joints is important to understand the functions of human joints[1],to assist in pathological diagnostics[2] and to monitor the actual three dimensional (3D) positions of bone segments during surgeries[3] (e.g. total hip arthroplasty[4] (THA), total knee arthroplasty[5, 6] (TKA)) and to assess the outcomes of treatments[7, 8]. Skeletal kinematic data may be used in motion analyses combined with biomechanical modelling, e.g. musculoskeletal models combined with inverse dynamics approaches[9, 10]. Hence, a valid representation of actual skeletal motion and an accurate skeletal kinematics estimation is important in the fields of orthopedic research and human motion analysis[11]. However, the fact is that human skeletal structures are not exposed to the outside environment but are surrounded by the soft tissues (muscles, fat, skin etc.). Therefore, an effective measuring technique that could directly or indirectly detect the motion of bone is necessary to monitor and trace the movements of bone segments underlying the skin surface[12, 13].

Currently, skin-mounted markers are widely used in human motion analysis to estimate the motions of bones by assuming no relative motions between the skin and bone[14]. However, this method is subject to soft tissue artifacts (STA) because the markers attached on the skin cannot represent the actual motions of underlying bone segments[15]. It has been reported that STA can cause measurement errors of markers up to $30 \mathrm{~mm}$ in the thigh[16]. The propagation of STA to knee joint kinematics has been reported to lead to average rotational errors of up to $4.4^{\circ}$ and $13.1^{\circ}$ and average translational errors of up to $13.0 \mathrm{~mm}$ and $16.1 \mathrm{~mm}$ for walking and cutting motions, respectively[17]. Although many researchers attempted to compensate for the STA by computer modelling[13, 18-24], no significant improvement has been found in previous studies[12].

With the development of medical imaging technologies, fluoroscopic systems have been utilized to capture high accurate joint kinematics in the prosthetic measurement for TKA patients[25-27]. However, high cost, cumbersome setup and limited field of view (FOV) impede routine usage in the clinical setting. Recently, several groups have been working on the development of mobile fluoroscopy systems[28-31]. Although using a robotic trolley or gantry carrying the fluoroscopic system following the movement of subject extends the FOV, the radiation exposure to the subject remains inevitable. Recently advanced four-dimensional (4D) MRI [32-34] and CT[35, 36] techniques have been reported to track the bone motion and to quantify the respective joint kinematics inside the scanners[32-34]. The disadvantages of this method are the limited FOV, limited sample rate and the inability to measure kinematics during daily activities. 


\section{A novel ultrasound tracking system to track in-vivo knee joint motions during walking and stair descent: a feasibility study}

Besides abovementioned image modalities, ultrasound serves as a non-invasive and nonradiative imaging method to observe the soft tissues and internal organs in various clinical applications[37]. In addition, ultrasound is also capable of detecting bone surfaces through multiple layers of soft tissues[38]. Utilization of an ultrasound transducer combined with a surgical navigation system to accomplish the intra-operative registration of bone segments has been reported in computer assisted orthopedic surgeries[39-41]. Due to its capability of detecting a bone surface under dynamic motions, the combination of multiple ultrasound transducers with conventional motion capture markers provides a new approach to estimate the $3 \mathrm{D}$ positions and orientations of bone segments and to quantify related joint kinematics. The bone detections (i.e. depths from the skin to bone) accompanied with corresponding spatial positions (3D coordinates of the ultrasound transducers) provide sufficient information to reconstruct the $3 \mathrm{D}$ bone motion per time frame without the effect of STA that exists in skin-mounted marker measurements. In-vitro validation of this concept has been investigated for the knee joint in a previous study[42], which showed a relative high accuracy on the estimated tibiofemoral kinematics. A comparative with a conventional skin-mounted marker based system has been conducted in a cadaveric setting. The ultrasound tracking system showed a rather good accuracy in estimated 3D bone positions and quantified sixdegree of freedom (6-DOF) joint kinematics (maximum root-mean-square (RMS) error 3.44 for rotations and $4.88 \mathrm{~mm}$ for translations). However, to evaluate the in-vivo capability of tracking knee joint motions during daily activities has not been performed yet.

The aim of this study was to demonstrate and assess the in-vivo capability of our proposed ultrasound tracking system when healthy subjects performed several daily activities, including treadmill walking at three different speeds and stair descent. We expected that kinematics alterations caused by different imposed gait parameters could also be identified by the ultrasound tracking system.

\subsection{Method}

\subsubsection{Participants}

Five subjects (five males; age $37 \pm 10$ years; height: $180 \pm 8 \mathrm{~cm}$; weight: $75.4 \pm 14.1 \mathrm{~kg}$ ) participated in this study. Although one subject had a meniscus operation four years ago, there was no influence and/or complaints on performing exercises reflecting daily activities as conducted during this experiment. The other subjects had no history of injury, treatment or disorder affecting knee and hip functions. All subjects gave written informed consent. Prior to the experiment, each subject had an MRI scan using a Philips INGENIA 3T (BEST, the Netherlands) with a voxel size of $0.5 \mathrm{~mm} \times 0.5 \mathrm{~mm} \times 1 \mathrm{~mm}$ at the radiology department of Academisch Medisch Centrum (AMC, Amsterdam, Netherlands). After the MRI scan, the 
A novel ultrasound tracking system to track in-vivo knee joint motions during walking and stair descent: a feasibility study

obtained MRI images were segmented manually to generate subject-specific geometrical surface models of the femur and tibia using Mimics 17.0 (Materialise N.V., Leuven, Belgium), which were exported in STL format. The femoral and tibial anatomical reference frames (ARF) were then defined based on obtained bone geometries using a method previously described[43]. Approval of this study was obtained from the Ethical committee at the Radboud University Medical Center (RUMC).

\subsubsection{Ultrasound tracking System}

The ultrasound tracking system consisted of a conventional motion tracking system and an ultrasound signal acquisition system. In this study, we used Visualeyez VZ4000v system (PTI Phoenix Technologies Inc., Vancouver, Canada) equipped with two trackers to provide spatial positioning (see Figure 6.1 and 6.2). The ultrasound signal and marker positioning information was collected and synchronized in the Diagnostic Sonar FI Toolbox (Diagnostic Sonar Ltd, Livingston, UK) with 2.3GHZ CPU (Intel Core i7-3610QE) and 8GB RAM with a custom acquisition program written by LabVIEW (National Instruments, Austin, Texas, US). 30 A-mode ultrasound transducers (7.5 MHz, focus at $3.5 \mathrm{~cm}$, Imasonic SAS, Voray / l'Ognon, France) and 27 active optical markers (tracked by Visualeyez system) were installed into custom ultrasound holders. The ultrasound holders cover various anatomical areas on the lower extremity, including ankle, middle shaft of tibia, tibial condyles, femoral epicondyles, middle thigh, greater trochanter (Figure 6.3). The ultrasound holders were designed in SolidWorks (Waltham, Massachusetts, USA)) and manufactured using Polyamide powder material in 3D printer (EOS Formiga P110, EOS GmbH, Krailling, Germany) to ensure high accuracy on their 3D geometrical structures for maintaining the strength, rigidity, and stability.

The US transducers attached to the customized ultrasound holders reproduce the necessary information (i.e. the 3D discrete point cloud) to reconstruct bone motion through the obtained raw ultrasound signals and spatial information. The detailed description of this processing can be found in our previous paper[42]. The yielded discrete point cloud was fed to a registration algorithm, using a modified weighted iterative closet point algorithm[44, 45] to get the transformations from the original geometrical surface models to the actual $3 \mathrm{D}$ positions and orientations of bone models in the laboratory coordinate system. The raw ultrasound signals from 30 (15 for the femur and 15 for the tibia) A-mode ultrasound transducers and the raw 3D coordinates of 27 optical markers were synchronized and recorded at an average sampling rate $45 \mathrm{~Hz}$. Thus the 3D discrete point cloud was reproduced in an average $45 \mathrm{~Hz}$ sample rate during experiment. The respective tibiofemoral kinematics were derived from the method based on the ISB recommendations[46, 47]. 


\section{A novel ultrasound tracking system to track in-vivo knee joint motions during walking and stair descent: a feasibility study}

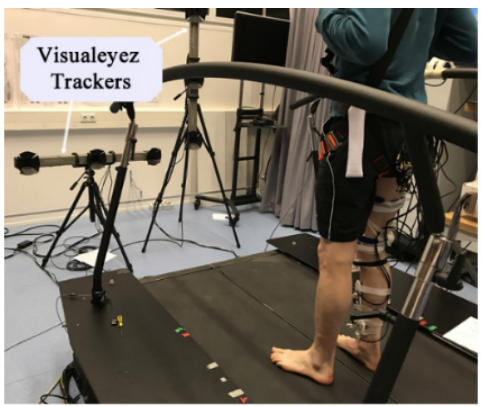

(a)

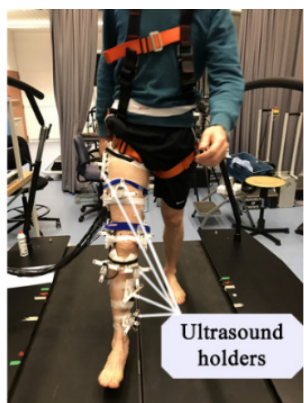

(b)

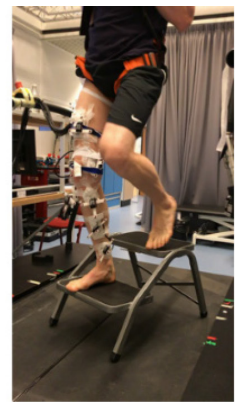

(c)

Figure 6.1 (a) a side view of experimental setup, including two Visualeyez trackers to track the optical markers on ultrasound holder; (b) a front view of experimental setup, one subject wore all ultrasound holder and performed a treadmill walking task; (c) a subject performed stair descent with the ultrasound tracking system measurement.
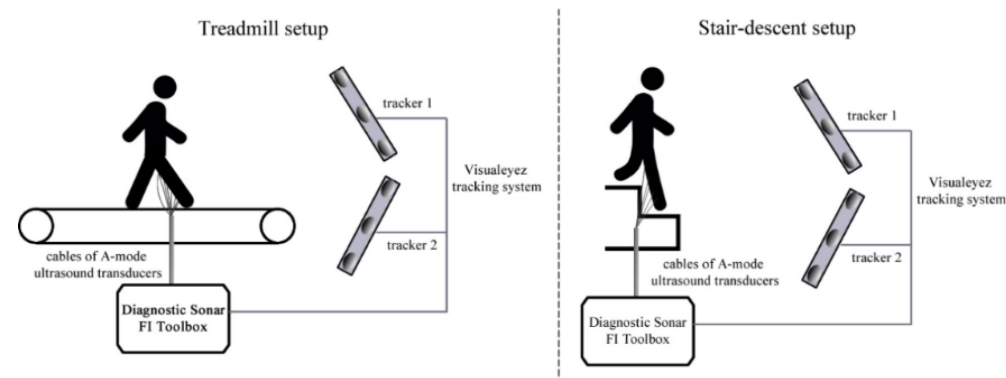

Figure 6.2 A schematic representation of the experimental setup for treadmill walking (left) and stair descent (right). Two Visualeyez trackers were used to record the spatial information of attached ultrasound holders. Diagnostic Sonar FI Toolbox received all raw ultrasound signals and was synchronized with collected spatial information from Visualeyez trackers.
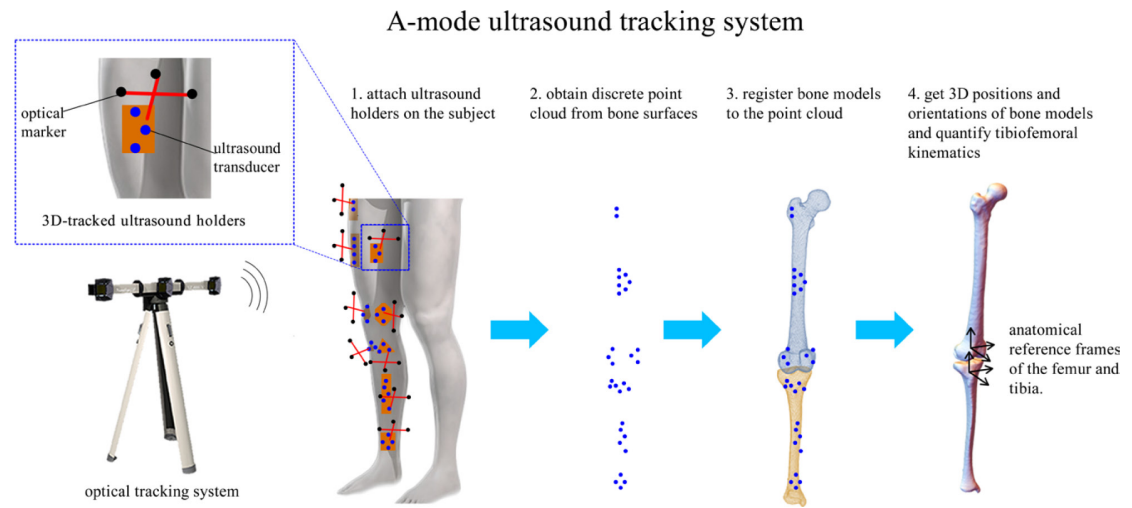

Figure 6.3 A schematic representation of the A-mode ultrasound system to quantify tibiofemoral kinematics from obtained point cloud. Also shown is the placement of ultrasound holders on the right leg. 
A novel ultrasound tracking system to track in-vivo knee joint motions during walking and stair descent: a feasibility study

\subsubsection{Experiments}

The ultrasound holders were attached to the right leg of each subject and were fixated by using skin tapes in order to cover all needed anatomical areas without any hindrance during movements. After attaching all ultrasound holders, each subject performed two sets of trials: 1) walking at three different imposed speeds $(1 \mathrm{~km} / \mathrm{h}, 2 \mathrm{~km} / \mathrm{h}$, and $3 \mathrm{~km} / \mathrm{h})$ on the treadmill as well as 2) stair descent from two consecutive stairs (first stair: $18 \mathrm{~cm}$ height; second stair: $21 \mathrm{~cm}$ height, next to the ground). For treadmill walking, at least five gait cycles were recorded for each trial. For the stair-descent trial, each subject was asked to repeat three times for stair-descent trial and was always asked to step the right leg at first for each stair. It took about one hour to complete an experiment of one subject, including attachment of ultrasound holders to the subject, the calibration procedure and all measurements of all trials.

\subsubsection{Data processing}

After all experiments, 3D knee joint motions and 6-DOF tibiofemoral kinematics were calculated for all trials over all gait cycles and three repeated stair-descent cycles. The calculated 6-DOF tibiofemoral kinematics of treadmill walking were averaged across five subjects under imposed three treadmill speeds. The mean and standard deviation across five subjects of calculated 6-DOF tibiofemoral kinematics for the stair-descent cycles were illustrated as the functions of percentage of two-stair descending (100\% represent one complete cycle of one-stair descending, thus completed cycle is $200 \%$ ).

To demonstrate the capability of detecting the bony surfaces from different anatomical areas and the capability of detecting the changes of depth (i.e. the thickness changes of the soft tissue layer) of detected bone surface caused by soft tissue deformation, several M-mode (motion mode) ultrasound images were generated. M-mode image is defined as motion display of the ultrasound wave along a chosen ultrasound line (in our case, a single ultrasound transducer element) during a time period. Its $\mathrm{x}$-axis represents the number of samples. Its $\mathrm{y}$ axis represents the intensity of received echo in a color map. It provides a two-dimensional view of the depth changes. We illustrated the M-mode images in two groups: 1) several anatomical areas at the same treadmill speed (lateral side of middle femur, anterior side of middle femur, femoral lateral epicondyle, medial side of middle tibia at $1 \mathrm{~km} / \mathrm{h}$ ) and 2) three different treadmill speeds of an identical location (lateral side of middle femur at 1, 2, 3, $\mathrm{km} / \mathrm{h})$.

\subsection{Results}

The mean of 6-DOF tibiofemoral kinematics across five subjects under imposed three different treadmill speeds are illustrated in Figure 6.4. The mean \pm standard deviation of 6- 


\section{A novel ultrasound tracking system to track in-vivo knee joint motions during walking and stair descent: a feasibility study}

DOF tibiofemoral kinematics across five subjects during stair descending is shown in Figure 6.5 .

\subsubsection{Treadmill walking}

The largest rotation motion was flexion-extension, followed by external-internal rotation and adduction-abduction. The peak knee flexion at the swing phase increased with increasing imposed speed. At heel-strike, the knee was not fully extended (reach $0^{\circ}$ ) at all three imposed speeds. As the imposed treadmill speed increased, the extension angle of the knee joint increased at heel-strike. The knee joint distraction started to increase from the heel-strike and reached the peak until the swing phase started. Walking at the lowest imposed speed resulted in the smallest range of motion (ROM) for all 6-DOF kinematics compared to a higher imposed speed.
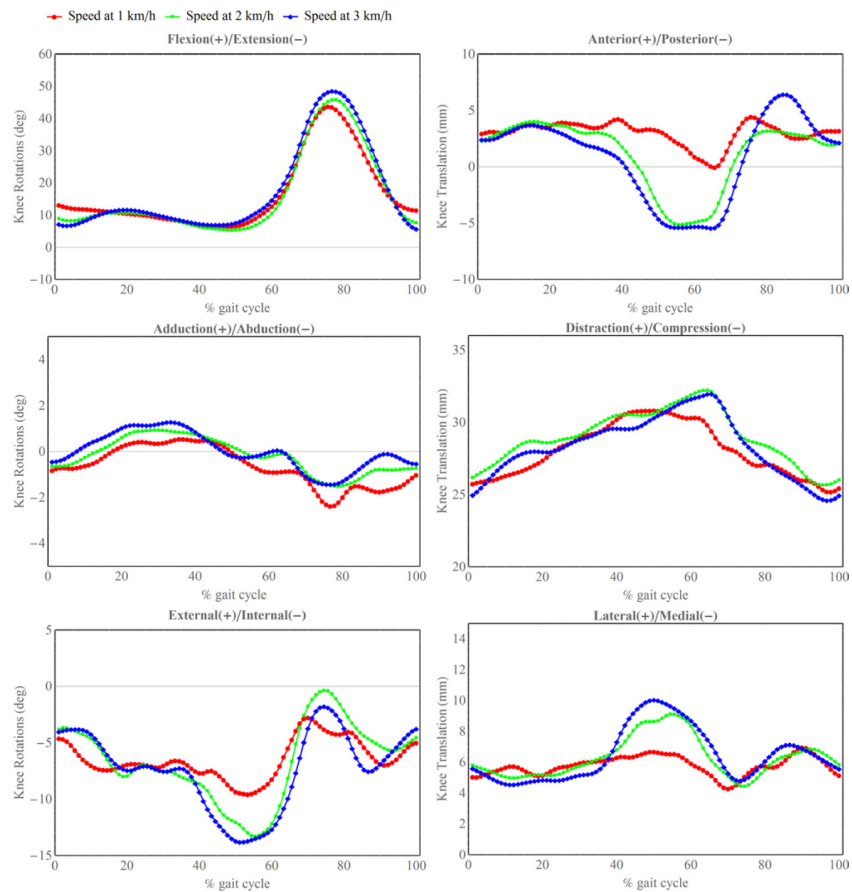

Figure 6.4 Averaged 6-DOF tibiofemoral kinematics across five subjects for imposed three different speeds: $1 \mathrm{~km} / \mathrm{h}$ (red line), $2 \mathrm{~km} / \mathrm{h}$ (green line), and $3 \mathrm{~km} / \mathrm{h}$ (blue line).

\subsubsection{Stair descending}

The mean flexion angle across five subjects reached the first peak $\left(35.1^{\circ}\right)$ during stepping down the first stair $(18 \mathrm{~cm})$ and reached the second peak $\left(41.0^{\circ}\right)$ during stepping down the second stair $(21 \mathrm{~cm})$. The knee joint distraction started to decrease when the right leg reached the next floor level and started to flex the knee to support the increasing pressure on the right 
A novel ultrasound tracking system to track in-vivo knee joint motions during walking and stair descent: a feasibility study

knee. When the contralateral foot reached the same floor, the joint distraction began to increase until flexion angle was as the same as neutral standing. The similar changing pattern of joint distraction happened during stepping down to the second floor level.

\subsubsection{M-mode images}

Examples of several M-mode images of one subject are illustrated in Figure 6.6. The level of thickness is logical at different anatomical areas, as thick around the middle of thigh, thin around the middle of shank. The depth changes at the same walking speed were different for various anatomical locations. The depth changing at the medial side of middle tibia was very small, which is logical as the soft tissue is the smallest (and therefore its expected deformation) compared to other locations. The anterior and lateral sides of middle femur had rather large variations in detected bone depths. Again, this is logical as the thickness of soft tissue (and its expected deformation during walking) is the larger than other locations. The frequency of depth change on the lateral side of middle femur increased with the increase of the imposed treadmill speed. The changing range of depth also increased with the increases of speed, suggesting more soft tissue deformation at higher walking speed.

\subsection{Discussion}

We presented a novel method to dynamically track the knee joint motion and to quantify 6-DOF tibiofemoral kinematics in a non-invasive and non-radiative manner. The combination of multiple A-mode ultrasound transducers with a conventional motion capture system provides an alternative method to capture skeletal motions and kinematics with mitigating the effect of STA. In this study, the in-vivo capability of our proposed ultrasound tracking system to measure knee joint motion and to quantify 6-DOF tibiofemoral kinematics was demonstrated in two motor tasks of daily activities. The kinematic alterations caused by different gait parameters has also been identified by the ultrasound tracking system. The peak flexion angle during swing phase on treadmill walking reduced when participants walked at the slow imposed speed, which is in accordance with the findings in a previous study[48]. Similarly, a smaller ROM was associated with a lower imposed speed during walking for all 6-DOF tibiofemoral kinematics[48]. The patterns of obtained 6-DOF tibiofemoral kinematics on the treadmill waking were in accordance with those of previously obtained tibiofemoral kinematics utilizing a mobile fluoroscopy system[49]. For the stair descending, the peak flexion angle was correlated with the height of the stair level. The kinematic alterations caused by small changes of gait parameters could be recognized by our ultrasound tracking system, which proves a certain extent of sensitivity of the ultrasound tracking system. 


\section{A novel ultrasound tracking system to track in-vivo knee joint motions during walking and stair descent: a feasibility study}
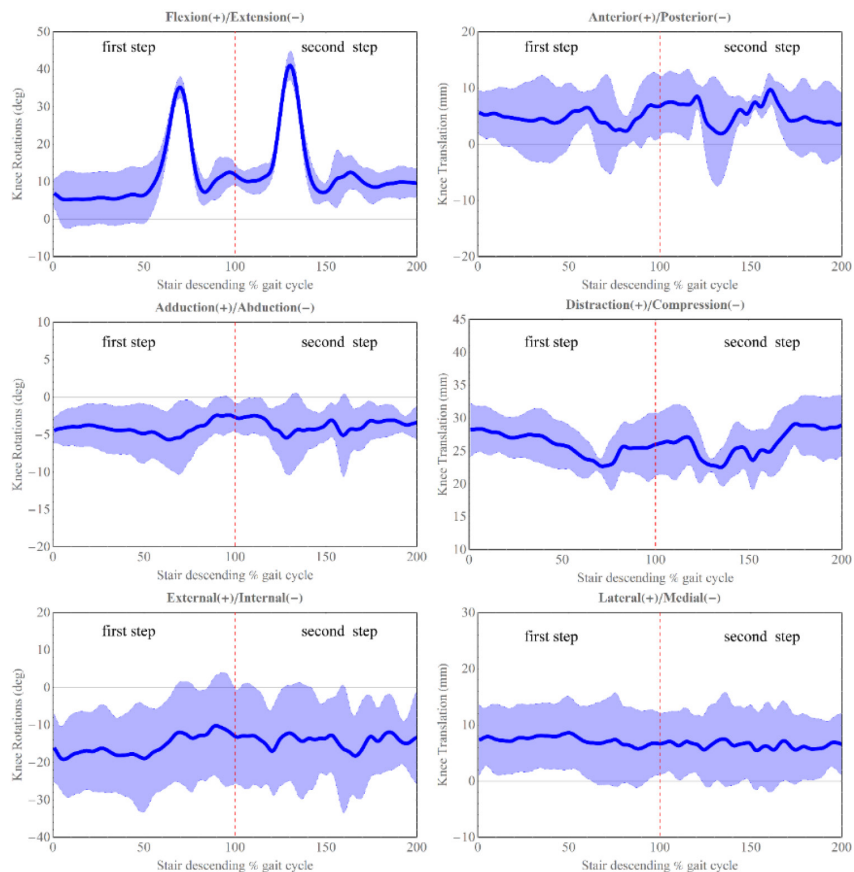

Figure 6.5 6-DOF tibiofemoral kinematics for two consecutive stairs descending across five subjects. The solid line represents the mean data while the shaded areas represent \pm 1 standard deviation from the mean. $100 \%$ of stair descending cycle represents the completion of the first floor. $200 \%$ of stair descending cycle represents the completion of the second floor.

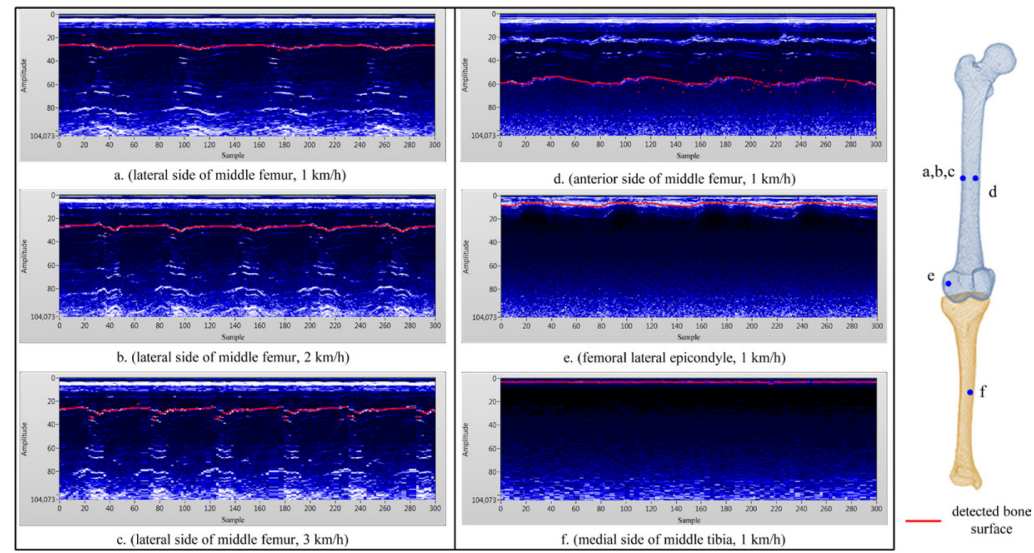

Figure 6.6 Left: the examples of six M-mode images of one subject: x-axis of M-mode image represents the number of samples, $y$-axis of $M$-mode image represents the intensity of received ultrasound echo in a color map; $(\mathrm{a}, \mathrm{b}, \mathrm{c})$ the M-mode images for lateral side of middle femur location at $1 \mathrm{~km} / \mathrm{h}, 2 \mathrm{~km} / \mathrm{h} 3 \mathrm{~km} / \mathrm{h}$ respectively; (d) the M-mode image for anterior side of middle femur at $1 \mathrm{~km} / \mathrm{h}$; (e) the M-mode image for femoral lateral epicondyle at $1 \mathrm{~km} / \mathrm{h}$; (e) the M-mode image for medial side of middle tibia at $1 \mathrm{~km} / \mathrm{h}$; right: the illustration of abovementioned anatomical locations on the femur and tibia. 


\section{A novel ultrasound tracking system to track in-vivo knee joint motions during walking and stair descent: a feasibility study}

The novelty of this study lies in the combined implementation of two existing techniques, i.e. motion capture and ultrasound imaging. Taking advantage of ultrasound techniques extends the range of detection of a conventional motion capture system from superficial skin surface tracking to internal bony surface tracking. As a consequence, the sufficient spatial information (trajectories) of bony segments under the skin surface contributes to the accurate bone motion tracking and accurate kinematic estimation.

Relative to a conventional skin-marker system, the ultrasound tracking system has the clear advantage of the reduction of STA on the measurement data and its propagation on kinematic outcomes[50]. As demonstrated in the examples of M-mode images, A-mode ultrasound transducers have the capability of detecting the depth changes of bone surfaces at different anatomical areas. This capability will improve the validity of representing actual bone movement, since the trajectories of bone surfaces will be measured instead of superficial skin surfaces. A comparison with a skin-mounted marker measurement in a cadaveric setting has been conducted in our previous study. However, a critical comparison with a skin marker system under in-vivo conditions is yet to be performed, particularly if a ground truth method (e.g. an advanced mobile fluoroscopic system)[28, 29] can be incorporated. Currently, the FOV of our system is the same as the conventional motion capture systems, since it only depends on the FOV of the employed motion capture system. In addition, the length of the cables connected to the ultrasound transducers also restricts the maximum dynamic motion range. However, this aspect can be solved reasonably easily by extending the length of cables or employing an ambulant acquisition terminal instead of a standalone desktop computer on the side.

This work has several limitations. Firstly, no "ground truth" measurement was employed during experiment. There is not a non-invasive and non-radiative method to obtain the ground truth of movements (walking and stair descent). Available methods like intra-cortical bone pins and fluoroscopic systems have obvious ethical restrictions. Even though, we propose that an in-vivo validation study will be completed in the near future so that the results would facilitate the improvements of the current system and provide valuable comparisons with existing techniques. Secondly, only five healthy subjects were involved in this study. Ideally, a cohort of living subject covering different patient and healthy groups with different sizes and BMIs accompanied with a ground truth measurements as a reference could provide more valuable information with regard to the pathological patterns on kinematics. Thirdly, it has been shown that gait patterns on a treadmill are different to freely normal level walking [49]. However, in this study, the focus was on the demonstration of knee joint motion tracking during dynamic movements and detecting the kinematic alterations caused by different imposed treadmill speeds and heights of staircase. Treadmill speed is a convenient parameter 


\section{A novel ultrasound tracking system to track in-vivo knee joint motions during walking and stair descent: a feasibility study}

to change. Fourthly, the cables and skin tapes may influence the nature gait pattern for individuals. In the future, we are aiming to develop a miniature and light-weight system towards a wearable measurement system that would facilitate its implementation in the clinic. Furthermore, future study will also focus on the improvement of designing the ultrasound holders to create a lighter, smaller, user friendly and ergonomic design. These improvements of the holders will also be beneficial to utilize our system in a broader application field and to facilitate clinical implementation.

In summary, we developed an ultrasound tracking system that is capable of capturing the soft tissue deformation and acquiring knee joint motion. Hence, we conclude that this prototyping system has great potential to measure human kinematics in an ambulant, nonradiative and non-invasive manner.

\section{References}

1. Ramsey, D.K. and P.F. Wretenberg, Biomechanics of the knee: methodological considerations in the in vivo kinematic analysis of the tibiofemoral and patellofemoral joint. Clinical Biomechanics, 1999. 14(9): p. 595-611.

2. Schilling, C., et al., The effect of design parameters of dynamic pedicle screw systems on kinematics and load bearing: an in vitro study. European Spine Journal, 2011. 20(2): p. 297-307.

3. Simon, D. What is "registration" and why is it so important in CAOS.

4. Sugano, N., et al., Accuracy evaluation of surface-based registration methods in a computer navigation system for hip surgery performed through a posterolateral approach. Comput Aided Surg, 2001. 6(4): p. 195-203.

5. Anderson, K.C., K.C. Buehler, and D.C. Markel, Computer assisted navigation in total knee arthroplasty: comparison with conventional methods. J Arthroplasty, 2005. 20(7 Suppl 3): p. 132-8.

6. Mavrogenis, A.F., et al., Computer-assisted navigation in orthopedic surgery. Orthopedics, 2013. 36(8): p. 631-42.

7. Kaiser, J.M., et al., Effect of Loading on In Vivo Tibiofemoral and Patellofemoral Kinematics of Healthy and ACL-Reconstructed Knees. The American Journal of Sports Medicine. 0(0): p. 0363546517724417. 
A novel ultrasound tracking system to track in-vivo knee joint motions during walking and stair descent: a feasibility study

8. Zeng, X., et al., Relationship between Kellgren-Lawrence score and 3D kinematic gait analysis of patients with medial knee osteoarthritis using a new gait system. Scientific Reports, 2017. 7(1): p. 4080.

9. Delp, S.L., et al., OpenSim: open-source software to create and analyze dynamic simulations of movement. IEEE Trans Biomed Eng, 2007. 54(11): p. 1940-50.

10. Gerus, P., et al., Subject-specific knee joint geometry improves predictions of medial tibiofemoral contact forces. Journal of biomechanics, 2013. 46(16): p. 2778-2786.

11. Fuller, J., et al., A comparison of lower-extremity skeletal kinematics measured using skin- and pin-mounted markers. Human Movement Science, 1997. 16(2-3): p. 219-242.

12. Richard, V., A. Cappozzo, and R. Dumas, Comparative assessment of knee joint models used in multi-body kinematics optimisation for soft tissue artefact compensation. $\mathrm{J}$ Biomech, 2017.

13. Andersen, M.S., et al., Do kinematic models reduce the effects of soft tissue artefacts in skin marker-based motion analysis? An in vivo study of knee kinematics. Journal of Biomechanics, 2010. 43(2): p. 268-273.

14. Lafortune, M.A., et al., Three-dimensional kinematics of the human knee during walking. Journal of Biomechanics, 1992. 25(4): p. 347-357.

15. Cereatti, A., et al., Standardization proposal of soft tissue artefact description for data sharing in human motion measurements. J Biomech, 2017.

16. Akbarshahi, M., et al., Non-invasive assessment of soft-tissue artifact and its effect on knee joint kinematics during functional activity. Journal of Biomechanics, 2010. 43(7): p. 1292-1301.

17. Benoit, D.L., et al., Effect of skin movement artifact on knee kinematics during gait and cutting motions measured in vivo. Gait \& Posture, 2006. 24(2): p. 152-164.

18. Bonnet, V., et al., Joint kinematics estimation using a multi-body kinematics optimisation and an extended Kalman filter, and embedding a soft tissue artefact model. Journal of Biomechanics, 2017.

19. Cappozzo, A., et al., Surface-marker cluster design criteria for 3-D bone movement reconstruction. IEEE Transactions on Biomedical Engineering, 1997. 44(12): p. 1165-1174.

20. Andersen, M.S., M. Damsgaard, and J. Rasmussen, Kinematic analysis of overdeterminate biomechanical systems. Comput Methods Biomech Biomed Engin, 2009. 12(4): p. 371-84. 


\section{A novel ultrasound tracking system to track in-vivo knee joint motions during walking and stair descent: a feasibility study}

21. Bonnechère, B., et al., Physiologically corrected coupled motion during gait analysis using a model-based approach. Gait \& Posture, 2015. 41(1): p. 319-322.

22. Charlton, I.W., et al., Repeatability of an optimised lower body model. Gait \& Posture, 2004. 20(2): p. 213-221.

23. Duprey, S., L. Cheze, and R. Dumas, Influence of joint constraints on lower limb kinematics estimation from skin markers using global optimization. Journal of Biomechanics, 2010. 43(14): p. 2858-2862.

24. Lu, T.W. and J.J. O'Connor, Bone position estimation from skin marker coordinates using global optimisation with joint constraints. Journal of Biomechanics, 1999. 32(2): p. 129-134.

25. Bingham, J. and G. Li, An Optimized Image Matching Method for Determining InVivo TKA Kinematics with a Dual-Orthogonal Fluoroscopic Imaging System. Journal of Biomechanical Engineering, 2006. 128(4): p. 588-595.

26. Baka, N., et al., Evaluation of automated statistical shape model based knee kinematics from biplane fluoroscopy. Journal of biomechanics, 2014. 47(1): p. 122-129.

27. Gray, H.A., S. Guan, and M.G. Pandy, Accuracy of mobile biplane X-ray imaging in measuring 6-degree-of-freedom patellofemoral kinematics during overground gait. Journal of Biomechanics, 2017. 57: p. 152-156.

28. Guan, S., et al., Mobile Biplane X-Ray Imaging System for Measuring 3D Dynamic Joint Motion During Overground Gait. IEEE Trans Med Imaging, 2016. 35(1): p. 326-36.

29. List, R., et al., A moving fluoroscope to capture tibiofemoral kinematics during complete cycles of free level and downhill walking as well as stair descent. PLoS One, 2017. 12(10): p. e0185952.

30. Mahfouz, M.R., et al., A robust method for registration of three-dimensional knee implant models to two-dimensional fluoroscopy images. IEEE Transactions on Medical Imaging, 2003. 22(12): p. 1561-1574.

31. Dennis, D.A., et al., In vivo determination of normal and anterior cruciate ligamentdeficient knee kinematics. Journal of Biomechanics, 2005. 38(2): p. 241-253.

32. Mazzoli, V., et al., Accelerated 4D self-gated MRI of tibiofemoral kinematics. NMR Biomed, 2017. 
A novel ultrasound tracking system to track in-vivo knee joint motions during walking and stair descent: a feasibility study

33. Clarke, E.C., et al., A non-invasive, 3D, dynamic MRI method for measuring muscle moment arms in vivo: Demonstration in the human ankle joint and Achilles tendon. Medical Engineering \& Physics, 2015. 37(1): p. 93-99.

34. Kaiser, J., et al., Measurement of 3D Tibiofemoral Kinematics using Volumetric SPGR-VIPR Imaging. Magnetic resonance in medicine : official journal of the Society of Magnetic Resonance in Medicine / Society of Magnetic Resonance in Medicine, 2013. 69(5): p. 1310-1316.

35. Forsberg, D., et al., Quantitative analysis of the patellofemoral motion pattern using semi-automatic processing of 4D CT data. Int J Comput Assist Radiol Surg, 2016. 11(9): p. $1731-41$.

36. Zhao, K., et al., A Technique for Quantifying Wrist Motion Using FourDimensional Computed Tomography: Approach and Validation. Journal of Biomechanical Engineering, 2015. 137(7): p. 0745011-0745015.

37. Smistad, E., et al., Medical image segmentation on GPUs - A comprehensive review. Medical Image Analysis, 2015. 20(1): p. 1-18.

38. Wein, W., et al., Automatic bone detection and soft tissue aware ultrasound-CT registration for computer-aided orthopedic surgery. International Journal of Computer Assisted Radiology and Surgery, 2015. 10(6): p. 971-979.

39. Fieten, L., et al., Fast and accurate registration of cranial CT images with A-mode ultrasound. Int J Comput Assist Radiol Surg, 2009. 4(3): p. 225-37.

40. Talib, H., et al., Information filtering for ultrasound-based real-time registration. IEEE Trans Biomed Eng, 2011. 58(3): p. 531-40.

41. Otake, Y., et al., Intraoperative image-based multiview 2D/3D registration for image-guided orthopaedic surgery: incorporation of fiducial-based C-arm tracking and GPUacceleration. IEEE Trans Med Imaging, 2012. 31(4): p. 948-62.

42. Niu, K., et al. A Novel Tibiafemoral Kinematics Measurement System Based on Multi-Channel A-Mode Ultrasound System. in CAOS 2017. 17th Annual Meeting of the International Society for Computer Assisted Orthopaedic Surgery. 2017. Aachen, Germany: EasyChair.

43. Miranda, D.L., et al., Automatic determination of anatomical coordinate systems for three-dimensional bone models of the isolated human knee. Journal of Biomechanics, 2010. 43(8): p. 1623-1626. 


\section{A novel ultrasound tracking system to track in-vivo knee joint motions during walking and stair descent: a feasibility study}

44. Maurer, C.R., Jr., R.J. Maciunas, and J.M. Fitzpatrick, Registration of head CT images to physical space using a weighted combination of points and surfaces. IEEE Trans Med Imaging, 1998. 17(5): p. 753-61.

45. Besl, P.J. and H.D. McKay, A method for registration of 3-D shapes. IEEE Transactions on Pattern Analysis and Machine Intelligence, 1992. 14(2): p. 239-256.

46. Wu, G. and P.R. Cavanagh, ISB recommendations for standardization in the reporting of kinematic data. Journal of Biomechanics, 1995. 28(10): p. 1257-1261.

47. Grood, E.S. and W.J. Suntay, A joint coordinate system for the clinical description of three-dimensional motions: application to the knee. J Biomech Eng, 1983. 105(2): p. 13644.

48. Mannering, N., et al., Three-dimensional knee kinematic analysis during treadmill gait: Slow imposed speed versus normal self-selected speed. Bone Joint Res, 2017. 6(8): p. 514-521.

49. Guan, S., et al., In vivo six-degree-of-freedom knee-joint kinematics in overground and treadmill walking following total knee arthroplasty. Journal of Orthopaedic Research, 2016: p. n/a-n/a.

50. Jia, R., et al., CAT \& MAUS: A novel system for true dynamic motion measurement of underlying bony structures with compensation for soft tissue movement. Journal of Biomechanics, 2017. 

General discussion and conclusions

\section{Chapter 7 General discussion and conclusions}




\section{General discussion and conclusions}

The goal of the research, as presented in this thesis, was to develop and validate a new method that is able to track skeletal motion in the knee joint and to quantify tibiofemoral kinematics under dynamic conditions. Our proposed method can be briefly described as:

1) multiple A-mode ultrasound transducers are combined with conventional optical markers in order to detect sufficient bony points covering the femur and tibia.

2) Subsequently, these obtained points are used to determine the unique $3 \mathrm{D}$ position and orientation of bone segments by a point cloud registration algorithm.

To achieve the goal of this study and to assess the feasibility of this new method, several research questions needed to be incrementally addressed, as described in Introduction (Chapter 1):

Q1. How many points are needed to determine the 3D position of a bone segment using this method and with what accuracy can this be obtained? How do the localization errors of the points affect the accuracy of segment position?

Q2. Is this method capable of determining the 3D position of a bone segment at a fixed position and with what accuracy?

Q3. Is it possible to quantify tibiofemoral kinematics at different static positions using this method and with what accuracy?

Q4. Is this method capable quantifying tibiofemoral kinematics dynamically in a cadaveric setting and with what accuracy?

Q5. How does this method compare to a skin marker system?

Q6. Is it feasible to quantify tibiofemoral kinematics in living subjects performing daily activities?

\subsection{Summary of research questions}

To answer Q1, a numerical simulation was conducted to explore the possibility of registering the detected point cloud with a known bone shape model in Chapter 2. Furthermore, the sensitivity of the number of acquired points and the sensitivity of induced noise were also investigated. To validate our registration method and to investigate the same parameters (number of points and magnitude of noise), a cadaveric experiment was also conducted to evaluate the registration method that is capable of determining a $3 \mathrm{D}$ position of bone segment at a fixed position (To answer Q2, also in Chapter 2). The results showed that our registration method (ICP with Perturbation Search) had a significant improvement on the registration accuracy, relative to the standard registration method, such as ICP [1]. The results 
of Chapter 2 furthermore proved the possibility of registering a known bone shape to a sparse point cloud and provided a guideline to decide the necessary number of A-mode ultrasound transducers that should be used in the first prototype system. We found that 15 points could get an acceptable accuracy (RMSE $<1.5 \mathrm{~mm}$ ) when localization error is low $(<1 \mathrm{~mm})$. Hence, we decided to employ $15 \mathrm{~A}$-mode ultrasound transducer for each bone segment. Thus a total number of $30 \mathrm{~A}$-mode ultrasound transducers were used for our final system.

To answer Q3, in Chapter 3, the principle of digitizing a bony point from a tracked Amode ultrasound probe was investigated in a cadaveric setting. The skeletal positions of shank and thigh were estimated while fixating the bones in a stationary position. We assumed that measuring multiple bony points covering the shank and thigh at one stationary pose was the same as the simultaneous measurement of bony points by multiple tracked A-mode ultrasound probes. Under these circumstances, the obtained bony points at one stationary pose were processed to quantify tibiofemoral 'kinematics' in a static manner. The derived kinematic outcomes were compared to the reference kinematics that were derived from inserted intra-cortical bone pins that we considered as the ground truth. The results derived from the tracked A-mode ultrasound probe showed a high accuracy in estimated rotations and translations. The results of this method showed the potential to estimate tibiofemoral kinematics under a dynamic situation after employing an array of tracked A-mode transducers covering the lower extremity at multiple locations. Hence, after this feasibility study it was decided to acquire the hardware to enable building a multi-channel A-mode system.

To explore the answer of $\mathbf{Q 4}$, we developed a multi-channel A-mode ultrasound tracking system as described in Chapter 4. The unique feature of our proposed ultrasound tracking system lies on the extension of the detection range of skin-mounted markers from the superficial measurement to the real bony surface. The combination of multiple ultrasound transducers (in our case, A-mode ultrasound transducers) with a conventional motion capture system improves the validity of estimating the $3 \mathrm{D}$ positions of the underlying bony segments which are required to accurately quantify tibiofemoral kinematics in a dynamic fashion. The results of Chapter 4 showed the high accuracy estimation in flexion-extension, adductionabduction. Although the reconstructed tibiofemoral kinematics were less accurate than those reported for fluoroscopic systems[2,3], it has the potential to overcome the effects of soft tissue artifacts of skin-mounted markers systems to produce more accurate kinematics. Thus, this advancement in measurement technology of true human motion shows to have great potential as a suitable skeletal motion capture and kinematic quantification method without the necessity of skin incisions or radiation exposure. 


\section{General discussion and conclusions}

To answer Q5, an in-situ comparison was conducted in a cadaveric experiment in order to investigate the performance of our method against the more traditional method using skinmounted markers (in Chapter 5). The ultrasound tracking system resulted, indeed, in more accurate 6-DOF kinematic estimations than skin-mounted markers. Consequently, this technique could be considered as an alternative method for measuring 6-DOF tibiofemoral kinematics, which may be adopted in gait analysis and clinical practice.

To find the answer of Q6, we aimed to demonstrate the feasibility of capturing skeletal motions and quantifying tibiofemoral kinematics for healthy subjects performing different daily activities (In Chapter 6). After changing several gait parameters, e.g. imposed speed of treadmill and height of staircase, kinematic alterations could be quantified which were in accordance with previous findings. Although it should be realized that there was no ground truth measurement accompanied in this experiment, the experiment was valuable at demonstrating some features of the system that are important for in-vivo assessment of human movement. The processing algorithms were modified to a weighted ICP method based on the amplitude of "confidence" of detected bone surface to obtain final kinematic outcomes [1] . Furthermore, we were successful in obtaining on-line (real time) kinematic data, which is important to directly assess the quality of the measurement. Finally, we demonstrated that the system prototype worked appropriately under in-vivo conditions and therefore has the potential to measure skeletal motion in research and clinical applications.

\subsection{Potential applications}

In section 1.2 of the introduction of this thesis, we defined accuracy requirements for various potential applications of this system:

- $\quad$ Gait analysis: $2^{\circ}$ and $2 \mathrm{~mm}[4]$.

- $\quad$ Prosthetic kinematics: $1^{\circ}$ and $1 \mathrm{~mm}[3,5,6]$.

- Surgical navigation: $1^{\circ}$ and $1 \mathrm{~mm}$ or below $2 \mathrm{~mm}$ registration accuracy $[7,8]$.

We did not succeed in generating a system that has the accuracy for these three application. However, we feel that there is great room to improve upon these errors. For example:

- enhanced registration algorithms can be developed that position the bony segment in a more accurate and robust manner;

- an improved motion capture system with higher inherent accuracy could be acquired and will reduce errors,

- ultrasound peak detection methods can be further optimized; 


\section{General discussion and conclusions}

- not all sensors are detecting bone at the same time thereby reducing the number of effective ultrasound sensors; more A-mode transducers than currently utilized could be utilized;

- the addition of one (or more) B-mode transducer(s) at vital positions could provide geometrical information to reduce errors.

We are convinced that we can generate next versions of the system in which some of the features as described above are implemented and which have accuracies as requested for the various applications.

\subsubsection{Gait analysis}

In gait analysis, skin-mounted markers are widely used to produce kinematic data. However, the validity of produced kinematic data and actual skeletal motion are affected by soft tissue artifacts (STA) that may result in kinematic errors up to more than $4^{\circ}$ in rotations and $10 \mathrm{~mm}$ in translations [9]. STA are generally generated and their effects are difficult to eliminate $[10,11]$, because the inherent mismatch between skin and bone movement is difficult to remove under all circumstances. Andersen et al. reported that the inclusion of optimized knee joint constraints did not eliminate or reduce the effects of STA and therefore does not improve the validity of reconstructed kinematics [12] for skin marker measurements.

As an alternative to conventional motion capture systems using skin-mounted markers, we introduced an ultrasound imaging technique into kinematic measurement, thus taking advantage of the capability of ultrasound transducers to detect the underlying skeletal structure through soft tissues. Hence, this can be regarded as an extended application of the navigated A-mode ultrasound probes utilized in computer assisted orthopedic surgery [1316]. The ultrasound tracking system thus has the potential to provide more accurate kinematics and to overcome the effects of STA, since the bone positions and orientations are estimated from the bony points instead of the positions of markers on the skin. The largest average errors of ultrasound tracking system were up to $2.46^{\circ}$ and $4.55 \mathrm{~mm}$ (Chapter 5), which showed to be more accurate kinematic estimations than respective skin markers measurement. Average rotational errors of $1.51 \pm 1.13^{\circ}$ (mean $\pm \mathrm{SD}$ ) and average translational errors of $3.14 \pm 1.72 \mathrm{~mm}$ (mean $\pm \mathrm{SD}$ ) were obtained in Chapter 4 . Hence, we did not fully reach the required accuracy for gait analysis.

Some researchers have proposed a similar concept by combining B-mode ultrasound transducers with a motion capture system[17, 18]. However, to our knowledge, dynamic 


\section{General discussion and conclusions}

tibiofemoral kinematic measurements have not been performed or validated in previous studies. Based on the current study, our proposed ultrasound tracking system shows the potential of being considered as an alternative to measure 6-DOF joint kinematics for gait analysis in the near future. In addition, no matter how the ultrasound holder shifts with respect to the underlying bone (i.e. either ultrasound holder to skin or skin to bone movements), as long as the underlying bone segment remains within the "field of view" of the ultrasound transducers, valid bony points can be captured and the registration algorithm could find an appropriate transformation to match the bone model to the point cloud of bony points and quantify 6-DOF tibiofemoral kinematics. When using skin-mounted markers, this type of shifting would contaminate the quality of skin-mounted marker dataset.

\subsubsection{Prosthetic kinematics}

Bi-planar fluoroscopic systems are considered as gold standard for prosthetic kinematic measurements with high accuracy of below $1^{\circ}$ and $1 \mathrm{~mm}$ errors in estimated kinematics. Single planar fluoroscopic systems are normally subject to the large errors in out-of-plane directions. Comparing our system to bi-planar fluoroscopic systems, only Flexion/Extension (RMS: $1.51^{\circ}$ ) and Abduction/Adduction (RMS: $0.88^{\circ}$ ) rotations are relatively comparable (see Chapter 4 ) with the accuracies of fluoroscopic system (below $1^{\circ}$ ). The other kinematic parameters have larger errors than mobile fluoroscopic systems [2, 19]. Therefore, to meet the required accuracy for prosthetic kinematic measurements, further study should be directed to improve the accuracy of the current system [2]. Compared to current fluoroscopic systems, the advantage of our ultrasound based skeletal motion capture method is the absence of radiation, smaller size and the possibility for generating a truly ambulant system.

\subsubsection{Surgical navigation}

Our current system could not fulfill the accuracy requirement for surgical navigation application. However, when these accuracies can be improved as discussed above, this system offers great potential to be adapted in a surgical navigation system. Especially in the field of computer assisted orthopedic surgery (CAOS). Replacing the inserted intra-cortical bone pins [8] will avoid the extra incisions and subsequent trauma to the patient (see Figure 7.1). 
Current method for surgical navigation

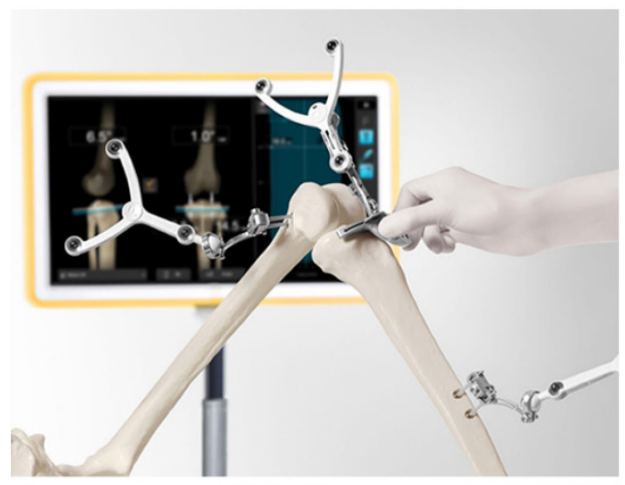

Intra-cortical bone pins (e.g. Brainlab)

(a)
Our proposal for surgical navigation

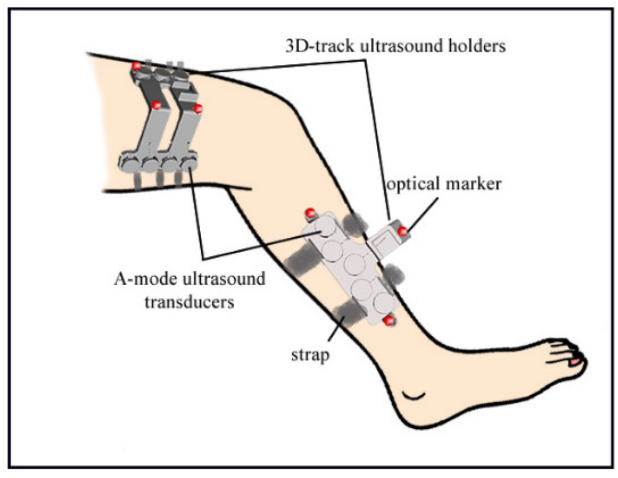

3D-tracked ultrasound holder

(b)

Figure 7.1 The comparison between current intra-cortical bone pins used in CAOS and multichannel A-mode ultrasound system. (a) A commercial application of 3D tracked intra-critical bone pins in TKA, example from Brainlab [20]. (b) our proposed multi-channel 3D-tracked Amode ultrasound system adapted for surgical navigation by reducing the number of transducers and optimizing its configurations with optical markers in order to determine the positions of the femur and tibia.

\subsubsection{Other potential applications}

The novelty of the method described in this thesis lies in the integration of existing techniques, i.e. ultrasound imaging and motion capture system, which broadens the range of detection of conventional motion capture systems, where skin markers record the trajectories of skin surfaces instead of skeletal surfaces. The characteristic of the ultrasound imaging technique makes the observation of skeletal surfaces from skin-attached sensors possible and effective. Therefore, this method can not only be applied in knee joint motion tracking, but could also be applied in other skeletal joints. We therefore anticipate that the current system can be transferred into applications such as the pelvic, ankle, elbow and shoulder skeletal joints.

Furthermore, the system could be transferred to measure deformations of non-skeletal structures. Since the ultrasound imaging is also capable of imaging other internal anatomical structures, the motion of all internal anatomical structures that are accessible to ultrasound imaging could be captured if its estimation is beneficial to clinical diagnostics and subsequent treatment. Obviously more sophisticated detection algorithms are required to detect various soft tissue structures within the body, but in principle, this is a technical challenge that can be overcome. 


\section{General discussion and conclusions}

\subsection{Limitations and Improvements}

Like any study or product, our current system has limitations as well. The largest limitation lies in the fact that the system has not yet been fully validated in-vivo on living subjects (with or without a movement disorder). The main problem with such an in-vivo validation lies in the absence of a non-invasive method to obtain the ground truth for the movement; available methods like intra-cortical bone pins and fluoroscopy obviously have serious ethical considerations. Even though we are not aware of any methods to obtain the ground truth noninvasively, we hope that an in-vivo validation study will be completed in the near future so that the results would facilitate the improvements of the current system and provide a valuable comparison with other existed (gold-standard) techniques.

Next to this main limitation, there are several areas where the system can be improved. First, the current design of the ultrasound holders only meet the requirements for laboratory experiments. Towards a clinical product, the ultrasound holders should be made lighter, smaller and more ergonomic. Improvements on these aspects are beneficial to make the measurement session easier for potential users and will facilitate application into practical, clinical departments. The next generation of ultrasound holders should be manufactured in a professional industry standard (e.g. $<0.2 \mathrm{~mm}$ error) to reach high accuracy and process. Second, the locations of the ultrasound holders on the lower extremity should be further optimized with regard to the number of A-mode transducers as well as the anatomical locations of the holders. A systematic test should be conducted to determine the most optimal anatomical locations in terms of skin contact, obtained bone signal and the influence on registration accuracy. As the first and second limitation are interrelated, they need to be addressed as one. Third, the registration algorithm used in this project was a weighted ICP based on the reliability and intensity of detected bone peak. This weighting allows compensating the instability of obtained bony points that is caused by missing optical markers or erroneously detected bone points from the ultrasound signal. Chapter 4 and 5 clearly showed its strength compared to other motion capture methods, the errors in the registration procedure still play a large part in the errors in the final outcomes (3D positions and kinematics). In future work, a more efficient registration algorithm should be developed to reach a higher accuracy than the current registration method, especially improving the robustness and stability while maintaining fast operation. Fourth, the overall volume and weight of the currently developed system cannot meet the requirements for ambulant measurements of all daily activities. An ambulant version of the system would be interesting in order to utilize this system in a broader field of research and in a clinical setting. Fortunately, the current hardware design has the possibility for miniaturizing the system into a backpack size for various daily activities. To ensure that the system remains accurate at 
movements at higher speeds and accelerations it can be expanded with inertial movement units (IMU's). These sensors are very accurate to quantify accelerations and decelerations. Fusion of information of the optical motion capture system, the IMU's and ultrasound detection information could lead to a more reliable and robust system with a broader application range (e.g. including sports activities).

\subsection{Final conclusions}

Ultrasound based skeletal motion capture is feasible and has the potential to achieve high accuracy in the estimation of skeletal motion and quantification of 6-DOF joint kinematics. The currently developed system showed the ability to overcome the effect of soft tissue artifacts that typically plague conventional motion capture systems, and showed the ability to mitigate the propagation of soft tissue artifacts into the final kinematic estimation. Therefore, this method could be considered as a suitable alternative to measuring human skeletal motion and if the accuracy could be improved with further research based on this thesis, could be applied in the future for human motion analysis in the fields of biomechanical research, prosthetic measurements, and surgical navigation. The method not only provides a new method to capture skeletal motion, but also has the potential to observe dynamic motion of all anatomical structures that are accessible to ultrasound imaging. Those potentials could further be explored in future work.

\section{References}

1. Besl, P.J. and H.D. McKay, A method for registration of 3-D shapes. IEEE Transactions on Pattern Analysis and Machine Intelligence, 1992. 14(2): p. 239-256.

2. Guan, S., et al., In vivo six-degree-of-freedom knee-joint kinematics in overground and treadmill walking following total knee arthroplasty. Journal of Orthopaedic Research, 2016: p. n/a-n/a.

3. Gray, H.A., S. Guan, and M.G. Pandy, Accuracy of mobile biplane X-ray imaging in measuring 6-degree-of-freedom patellofemoral kinematics during overground gait. Journal of Biomechanics, 2017. 57: p. 152-156.

4. Richard, V., A. Cappozzo, and R. Dumas, Comparative assessment of knee joint models used in multi-body kinematics optimisation for soft tissue artefact compensation. $\mathrm{J}$ Biomech, 2017.

5. Bingham, J. and G. Li, An Optimized Image Matching Method for Determining InVivo TKA Kinematics with a Dual-Orthogonal Fluoroscopic Imaging System. Journal of Biomechanical Engineering, 2006. 128(4): p. 588-595. 


\section{General discussion and conclusions}

6. Dennis, D.A., et al., In vivo determination of normal and anterior cruciate ligamentdeficient knee kinematics. Journal of Biomechanics, 2005. 38(2): p. 241-253.

7. Bae, D.K. and S.J. Song, Computer Assisted Navigation in Knee Arthroplasty. Clinics in Orthopedic Surgery, 2011. 3(4): p. 259-267.

8. Mavrogenis, A.F., et al., Computer-assisted navigation in orthopedic surgery. Orthopedics, 2013. 36(8): p. 631-42.

9. Benoit, D.L., et al., Effect of skin movement artifact on knee kinematics during gait and cutting motions measured in vivo. Gait \& Posture, 2006. 24(2): p. 152-164.

10. Cappozzo, A., et al., Position and orientation in space of bones during movement: anatomical frame definition and determination. Clin Biomech (Bristol, Avon), 1995. 10(4): p. 171-178.

11. Cappozzo, A., et al., Position and orientation in space of bones during movement: experimental artefacts. Clinical Biomechanics, 1996. 11(2): p. 90-100.

12. Andersen, M.S., et al., Do kinematic models reduce the effects of soft tissue artefacts in skin marker-based motion analysis? An in vivo study of knee kinematics. Journal of Biomechanics, 2010. 43(2): p. 268-273.

13. Chang, T.C., et al., A-Mode Ultrasound Bone Registration for Computer-Assisted Knee Surgery: Calibration and Robustness Test, in 25th Southern Biomedical Engineering Conference 2009, 15 - 17 May 2009, Miami, Florida, USA, A. McGoron, C.-Z. Li, and W.C. Lin, Editors. 2009, Springer Berlin Heidelberg. p. 97-100.

14. Amstutz, C., et al., A-mode ultrasound-based registration in computer-aided surgery of the skull. Arch Otolaryngol Head Neck Surg, 2003. 129(12): p. 1310-6.

15. Heger, S., et al., [Visual user guidance for registration based on A-mode ultrasound]. Biomed Tech (Berl), 2002. 47 Suppl 1 Pt 1: p. 61-4.

16. Fieten, L., et al., Fast and accurate registration of cranial CT images with A-mode ultrasound. Int J Comput Assist Radiol Surg, 2009. 4(3): p. 225-37.

17. Jia, R., et al., CAT \& MAUS: A novel system for true dynamic motion measurement of underlying bony structures with compensation for soft tissue movement. Journal of Biomechanics, 2017.

18. Masum, M.A., et al., Accuracy assessment of Tri-plane B-mode ultrasound for noninvasive 3D kinematic analysis of knee joints. Biomed Eng Online, 2014. 13: p. 122.

19. Guan, S., et al., Mobile Biplane X-Ray Imaging System for Measuring 3D Dynamic Joint Motion During Overground Gait. IEEE Trans Med Imaging, 2016. 35(1): p. 326-36.

20. BrainLab. KNEE3. [cited 2017 11.22.2017]; Available from: https://www.brainlab.com/en/surgery-products/orthopedic-surgery-products/kneenavigation-application/. 
Supplementary technical details

\section{Chapter 8 Supplementary technical details}




\section{Supplementary technical details}

\subsection{Overview}

The working principle of our final ultrasound tracking system which has been described in the previous chapters is illustrated in Figure 8.1. In this chapter, supplementary technical details regarding the design of our final system are introduced in several sections below:

- A-mode ultrasound transducer

- Ultrasound generation and acquisition

- Ultrasound holder design

- Software architecture

\subsection{A-mode ultrasound transducer}

\subsubsection{Frequency}

One of the most important aspects of the transducers is their transmitting frequency. The conflicting properties that we have to deal with are the axial resolution, which is better for higher frequencies, and the depth at which bone can still be seen which is better for lower frequencies, as high frequency ultrasound is easily absorbed and dispersed within the soft tissue. The amplitude of the received waveform is limited by the used equipment, and the safety regulations (voltage).

Since there is a thick soft tissue layer to go through at the thigh, we opted for a transducer frequency that is mostly used for visualizing internal organs (e.g. bowel) or deep muscles in clinical practice: the range from 5-10MHz. An A-mode ultrasound transducer with a resonant frequency of $7.5 \mathrm{MHz}$, would yield a wavelength of:

$$
\lambda=\frac{1480[\mathrm{~m} / \mathrm{s}]}{7.5 \times 10^{6}[\mathrm{~Hz}]}=197[\mu \mathrm{m}]
$$

The Barker code (see section 8.3.1) gives a sharp peak of the width of one period of the frequency with which it is encoded, so the wavelength can be seen as an approximation of the resolution. The ultrasound system samples (and generates waveforms) at 50MS/s. A much higher frequency than $7.5 \mathrm{MHz}$ would also yield problems with phase detection, as the reconstructed waveform would consist of fewer and fewer samples per excitation sine wave.

\subsubsection{Beam width}

The first tests were performed with commercially available Olympus transducers (V121$\mathrm{RM}$, outer diameter $10 \mathrm{~mm}$, element diameter $6.35 \mathrm{~mm}$, Olympus Corporation, Shinjuku, Tokyo, Japan) with a specified $7.5 \mathrm{MHz}$ peak frequency. When experimenting with the 


\section{Supplementary technical details}

transducers, the peak frequency was found to be $6.8 \mathrm{MHz}$, which was used as frequency for experiments with these transducers.

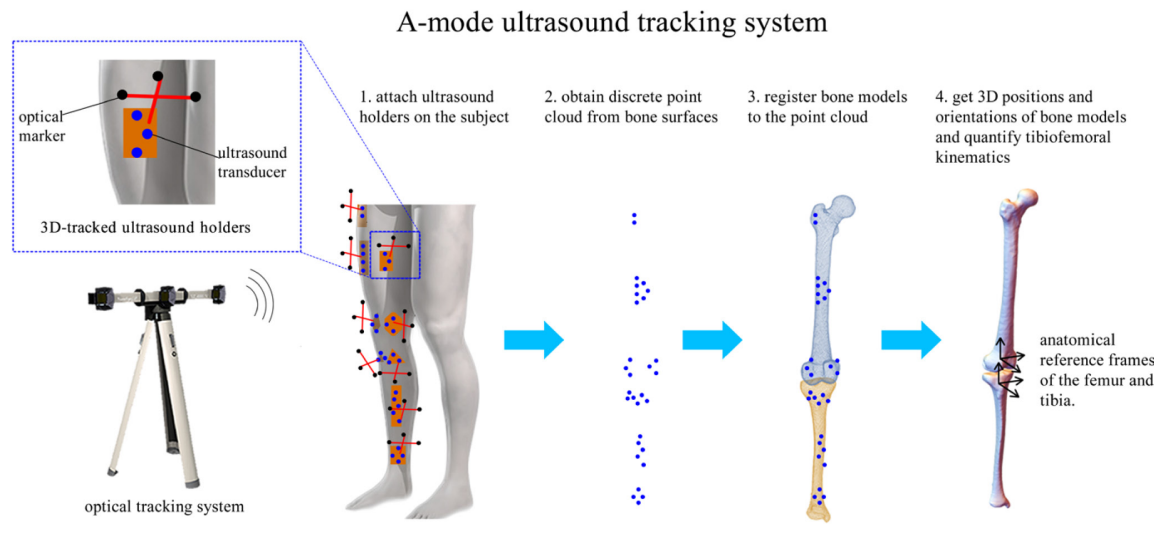

Figure 8.1 A schematic representation of the A-mode ultrasound system to quantify tibiofemoral kinematics. From left to right: camera tracks holders with A-mode transducers, with known 3D relation between holders and transducers a point cloud per bone can be obtained. Subject specific $3 \mathrm{D}$ bone shapes can be fitted to these point clouds. From these registered bones, the joint kinematics can be determined.

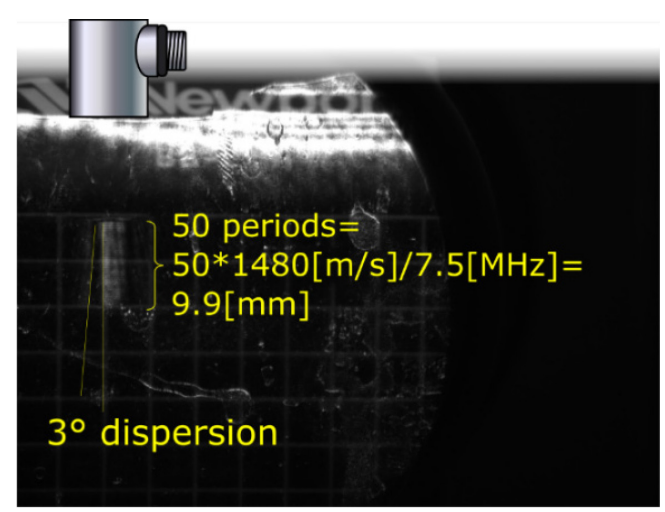

Figure 8.2 Schlieren picture of beamforming where 50 sine periods of $7.5 \mathrm{MHz}$ were transmitted. The formula calculates the total length of the burst using the speed of sound in water $(1480 \mathrm{~m} / \mathrm{s})$. The transducer (illustrated in place for clarity) has a diameter of $8 \mathrm{~mm}$. Below the transducer the pressure gradient due to a burst of 50 periods can be seen, comprising of a main lobe and two side lobes, the dispersion can mostly be seen in the side lobes.

Transducers can be focused, but the Olympus transducers were 'non-focused', both for simplicity and cost considerations. In our system approach we use a detected bone location as a single coordinate, but in reality we find a bony reflection which is within an ultrasound beam. To determine the dispersion of the beam we did a synchronous Schlieren [1] test and 


\section{Supplementary technical details}

determined that the dispersion of the waveform was $\sim 3^{\circ}$ in the near field (Figure 8.2). Hence, we can safely assume that the reflection that we measure is in a spot of roughly the size of the transducers.

When a larger system was constructed, 30 transducers were needed. Olympus could not provide us with axial beam profiles of their transducers, also other requirements such as MRI compatibility and subject safety (no electrically conductive parts) could not be accommodated. A partner was found in Imasonic (l'Ognon, France), who provided us first with samples, then with a set of 30 transducers. These transducers appeared to be more efficient; a comparative measurement showed a factor of 15 fold increase in returned signal amplitude with respect for the Olympus transducer for the same waveform and amplitude.

Although a non-focused transducer was ordered, the manufactured transducer was slightly focused at $35 \mathrm{~mm}$, as can be seen in Figure $8.3 \mathrm{a}$ and b. For frequency characteristics, in Figure 8.4 the time and frequency response of one of the transducers can be seen. Peak frequency is $7.5 \mathrm{MHz}$, with $5 \mathrm{MHz}$ bandwidth for all transducers that were acquired at Imasonic.

a

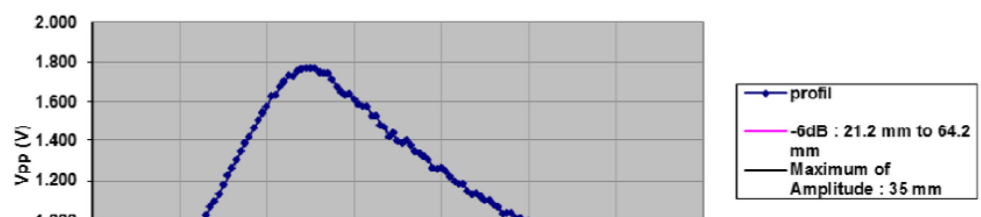

b
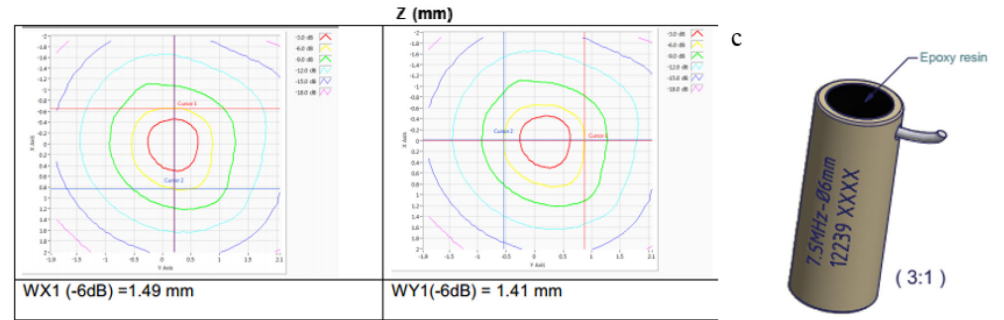

Figure 8.3 (a) Ultrasound pressure over axial distance. Intensity is highest at $35 \mathrm{~mm}$, this also means that the beam is narrowest at $35 \mathrm{~mm}$ (horizontal axis is depth; vertical axis is returned signal amplitude for a fixed excitation. Apparently the transducer is focused at $35 \mathrm{~mm}$ ). (b) Radial height and width of the beam at the focal point $(35 \mathrm{~mm}$ ); at the $-6 \mathrm{~dB}$ (half intensity) point, the width of the beam is approximately $1.4 \mathrm{~mm}$. (c) Physical design of transducer, outside diameter is $8 \mathrm{~mm}$. 


\section{Supplementary technical details}

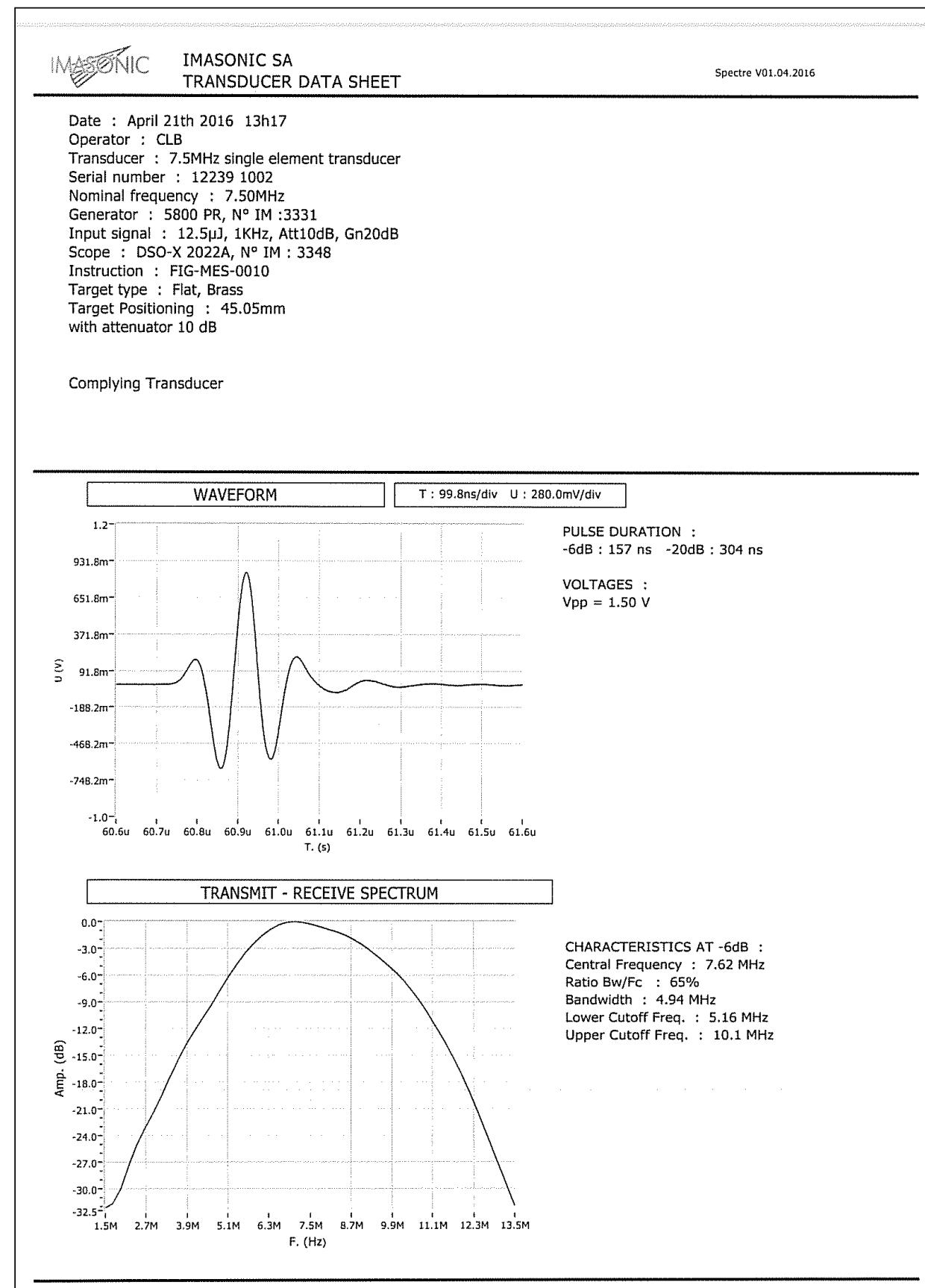

Figure 8.4 Test report for one of the transducers produced by Imasonic. 


\section{Supplementary technical details}

\subsubsection{Axial sensitivity test}
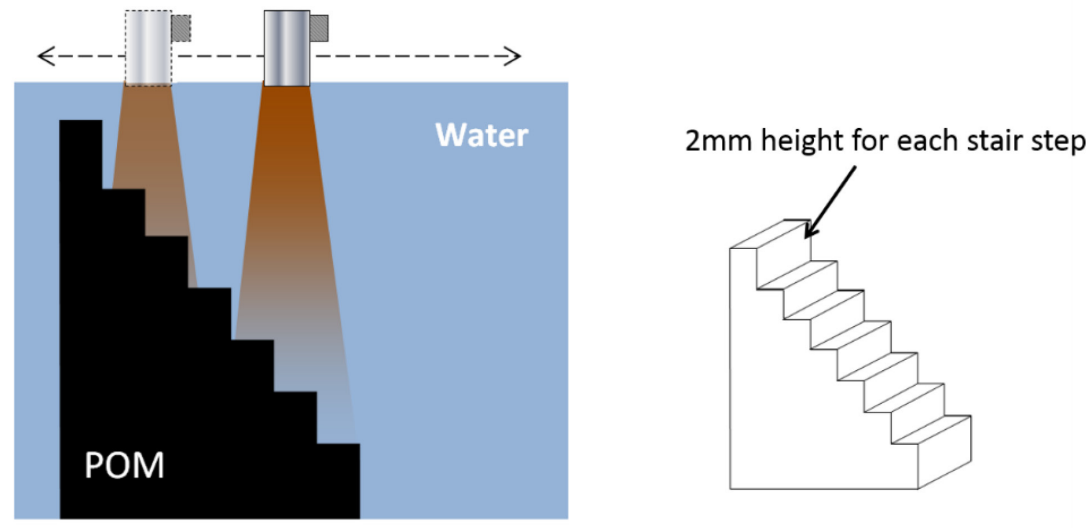

Figure 8.5 A schematic of the axial sensitivity setup for A-mode ultrasound. For clarity of the illustration, not all 24 steps are shown.

To evaluate the axial sensitivity of an A-mode ultrasound transducer, we performed a phantom study to assess the axial sensitivity of A-mode ultrasound measurement (Figure 8.5), this was done with the Olympus transducers. A 'staircase' was milled from Polyoxymethylene (POM) material with 24 stair steps (2 mm height for each step). The customized staircase was immersed in water. The A-mode ultrasound transducer was mounted on an X-Y table to be able to move along the $\mathrm{X}$-axis of $\mathrm{X}$-Y table step by step $(0.2$ $\mathrm{mm})$. For each step, a received ultrasound signal was recorded. The results as shown in Figure 8.6 were obtained by processing the recorded data to determine the detected depth for each acquisition. As the results show, the A-mode ultrasound transducer is capable of detecting the different depths and is sensitive to the depth changes. The differences between two adjacent detected depths were calculated, as is shown in Figure 8.7. As the height of stair step is $2 \mathrm{~mm}$, the calculated differences of depth had three values, -2 (two adjacent points lied on different stair steps, the first point lied lower than last one), 0 (two adjacent points lied on same stair step), 2 (two adjacent points lied on different stair steps, the first point lied higher than last one). After processing, there were 24 stair steps recognized in total, which was in accordance with the actual number of stair steps. 


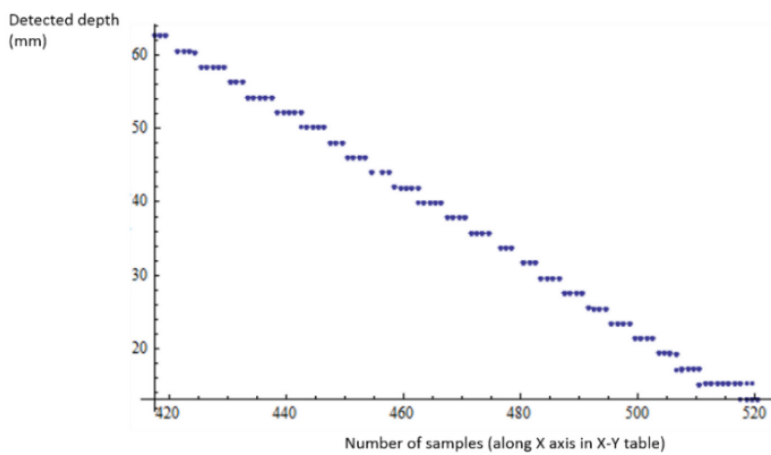

Figure 8.6 The detected depths for the stair phantom study.

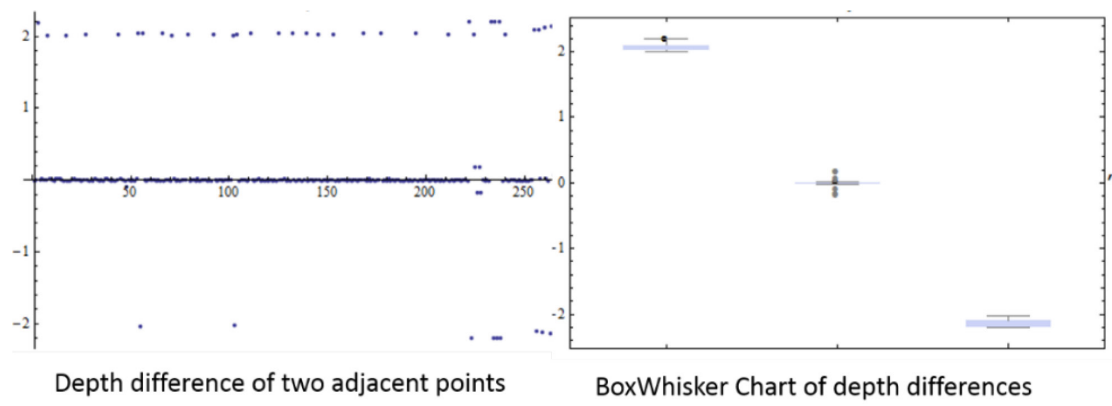

Figure 8.7 The results of data analysis on the depths found in the stair phantom study. Left: depth difference between two adjacent points on the X-axis, right: box-whisker chart of these values.

\subsubsection{Ultrasound Safety}

Ultrasound is known as a 'safe' technique, but some restrictions in the amount of mechanical power applied to the subject are present. The most important of these restrictions is the Mechanical Index (MI). In commercial ultrasound machines for clinical use, the MI is shown to give the operator an indication of the power that is transmitted. The MI is based on the Peak Negative Power; a high negative pressure could cause cavitation (small air bubbles due to local vacuum) in the blood.

The initial tests were all prepared with a single transducer. When the multiple transducer setup was built, a better understanding of the 'Mechanical Index' (MI) that is used as an indicator of subject safety was needed. The MI is determined as follows:

$$
M I=\frac{P N P}{\sqrt{f_{c}}}
$$




\section{Supplementary technical details}

Where $P N P$ is the peak negative pressure in $\mathrm{MPa}$, derated by $\frac{0.3 \mathrm{~dB}}{\mathrm{~cm} \cdot \mathrm{MHz}}$. The limit set by the FDA is that the MI should be lower than 1.9 [2]. With a calibrated needle hydrophone measurements were taken of the pressure that was created by our transducers, using different settings (Figure 8.8). Settings that were varied (with bold the setting that gave the highest $P N P)$ :

Maximum transmit voltage $(30,60,90, \mathbf{1 2 0})$

Barker code length ( 5 or 7 bit)

Duty cycle of the waveform $(40 \%$ and $\mathbf{8 0 \%})$

Frequency $(6.8 \mathrm{MHz})$

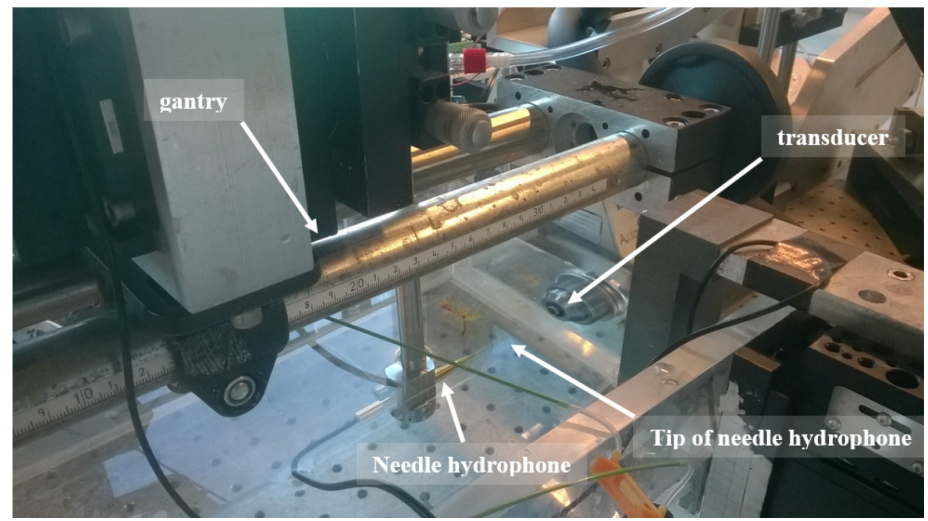

Figure 8.8 Needle Hydrophone setup. A needle hydrophone suspended from a cart on a gantry was submerged in a water tank. One transducer was placed in a hole in the water tank. The zaxis of both transducer and hydrophone were aligned manually. Measurements were taken at several depths along the transducer $\mathrm{z}$-axis.

The graph below (Figure 8.9) depicts the MI for the worst case setting. The measured peak negative pressure (absolute value given) is shown in blue, this pressure is derated with $\frac{0.3 \mathrm{~dB}}{\mathrm{~cm} \cdot \mathrm{MHz}}$ in the orange graph. The derived $\mathrm{MI}$ is shown in grey (value on right vertical axis). The highest value is 0.26 for a single transducer, which is far below the limit of 1.9. In the 'real' setup we are using $60 \mathrm{~V}$ excitation voltage, which yields an even lower MI. 


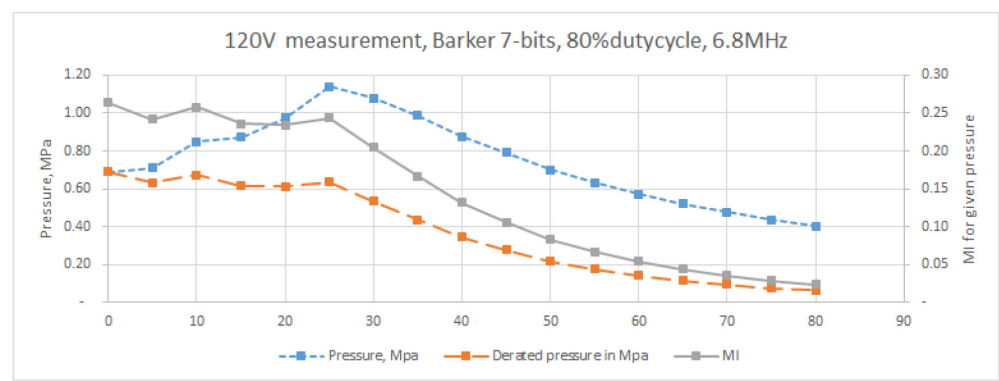

Figure 8.9 Highest Mechanical index is next to the transducer, where the MI is 0.26 . This is far below the limit of 1.9 as quantified by the FDA. Horizontal axis is in mm distance from transducer.

This result would mean that the FDA limit would be reached when more than 7 transducers would hit the same spot of tissue at the same time, in phase. This is highly unlikely, so we are certain that our system is safe for use on human subjects.

\subsection{Ultrasound generation and acquisition}

\subsubsection{Coded Excitation}

To detect a tissue-bone boundary, we are looking for good axial resolution. When sending a single period sine wave, the signal-to-noise ratio can be very low, and reflections can be missed. Sending longer waveforms will give higher SNR, but will make it difficult to find the exact center of the peak. Correlation of the pulsed waveform with the received one gives a broad peak for a sine wave $[3,4]$.

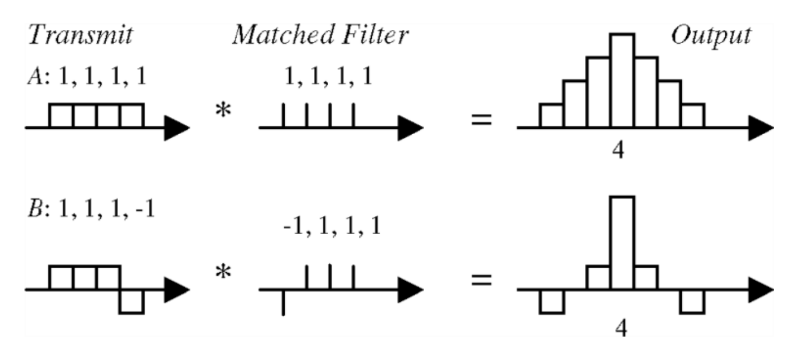

Figure 8.10 Comparison of 4-chip uncoded (top) and Barker coded (bottom) pulse sequence. The horizontal axis is the time, and the vertical axis is the amplitude. The encoding of the signal is in the phase, where +1 is encoded as one period of $\sin (x)$, and -1 is encoded as one period of $-\sin (\mathrm{x})$. (C) 2005 IEEE, source:[3].

Another option is to use a Barker code, this code gives a clear peak in the center of the auto-correlation. Barker code are codes that have the property that they consist of " +1 " and "-1" values and that the autocorrelation leads to a sharp peak in the center of the autocorrelation waveform. To use this in ultrasonography, the waveform is phase encoded, 


\section{Supplementary technical details}

as shown in Figure 8.10. A limited set of barker codes is available, the maximum length is 13 bit.

A third option that was evaluated was Golay coding (Figure 8.11). In this coding scheme, two waveforms are sent and auto correlated. The sum of the auto correlated waveforms shows a clear peak at the center, with phase encoding 2 small negative pulses are found near the peak, but no signal in the rest of the waveform. The clear downside of this approach is that the bandwidth of the system is halved; for every waveform that needs to be decoded the previous (other) waveform is also needed. In our first setups the frame rate was limited to the speed at which we could get the ultrasound data from the analog front ends into our program. This was especially tricky when looking at moving objects, because the two reflections would not overlap anymore. Although Golay yielded (slightly) better results in our preliminary setup, we chose to use Barker coding for the real setup in order to be independent of previous transmissions. With this setup we did the first test in combination with the optical tracking, and found it to be good enough to proceed to a full system.

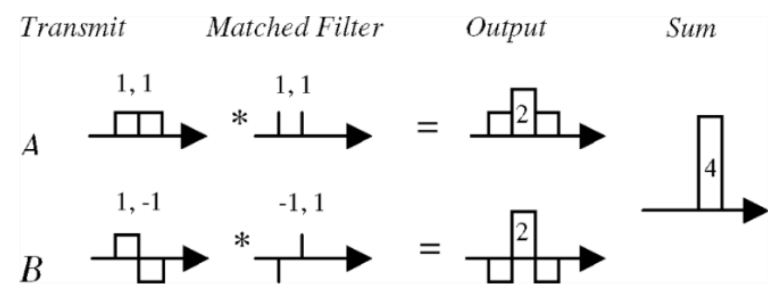

Figure 8.11 Illustration of Golay code that compresses without range sidelobes. Two transmissions with same target-transducer distance are needed for this to work. (C) 2005 IEEE, source:[3].

\subsubsection{Diagnostic Sonar setup}

Several systems were considered to use as a framework to program our own device: Siemens S2000, Verasonics, DiagnosticSonar, Adlink, Esaote and Cephasonics. Because previous experiments indicated that it was very beneficial to be able to create our own waveforms we looked for a machine that could provide this. Cephasonics (USA) and DiagnosticSonar (Livingston, UK) were both in an acceptable price range and met the requirements. DiagnosticSonar was chosen based on technical factors (higher sample rate, possibility to change 'core' LabVIEW functionality) and based on our need for technical support (very responsive, only one hour time difference). Also, the possibility to extend the National Instruments (Austin, TX, USA) PXI rack with other cards was regarded as beneficial. This was proven to be true, because both the Visualeyez cameras needed a $1 \mathrm{Mbps}$ serial link, which was achieved using a NI8432 card which was bought several months after the platform was delivered. 
The final system is a research ultrasound machine by Diagnostic Sonar (Livingston, UK), based on National Instruments (Austin, TX, USA) equipment. 30 transducers were purchased from Imasonic, and attached to this system. 3D localization information was obtained by measuring active marker positions with the Visualeyez system (PTI Phoenix, Canada).

The machine that was bought has these specifications:

- PXI chassis

- $\quad$ NI PXIe-8135 Core i7-3610QE 2.3GHz controller, 8GB RAM

- 32 channel transmit $(50 \mathrm{MS} / \mathrm{s})+$ multiplexer (max 64 transmit)

- 32 channel receive $(50 \mathrm{MHz})+$ multiplexer $(\max 64$ receive)

- Maximum pulse voltage: $\pm 120 \mathrm{~V}$

- Connector: ITT Cannon 156-pin ZIF

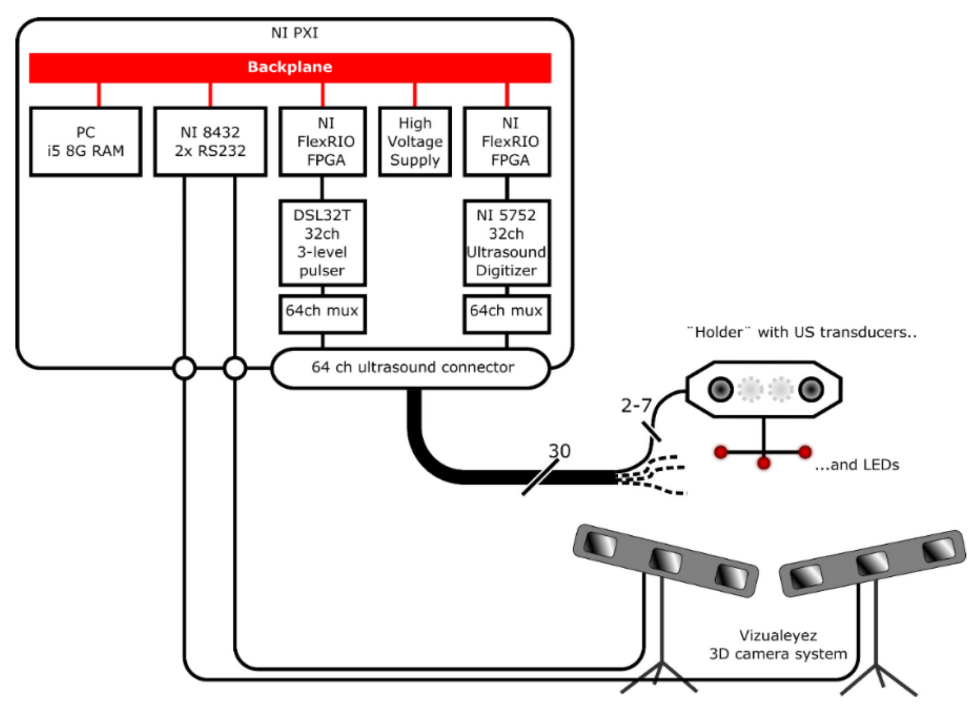

Figure 8.12 A schematic overview of the hardware for the ultrasound and 3D marker acquisition

A schematic of the Diagnostic setup can be found in Figure 8.12. Both the pulser as the receiver are capable of interfacing to 32 channels. Multiplexers were added to be able to drive more than 32 transducers in future, with a maximum of 64 transducers.

The Diagnostic Sonar setup has a main application that controls the FPGAs and sets up data transfer to the PC. This application can be run as stand-alone executable and be interfaced using DLLs provided by Diagnostic Sonar. We decided to run the application as a VI (Virtual Instrument) from the LabVIEW environment so we could add debugging methods to the code ourselves and add features that were not yet present in the compiled executable. 


\section{Supplementary technical details}

This main application is called "FITStream_FRD". From this main application, a setup can be made specifically to a certain setup: single element transducers, arrays with standard Bmode view and C-scan pictures. For our setup we can make a general setup for 30 transducers where we get 'live graphs' of the signal received on these transducers. By default the configuration only uses "pulse-receive" mode, so 30 responses would be acquired. We use a feature called "Full Raw Data" (FRD) in which every response from every transducer is received. A clear description of Full Matrix Capture and Full Raw Data is described in [5, 6] and for their original use in NDT.

\subsubsection{Data reduction}

When reading all channels, and every transducers' response to all channels, we would have to send a waveform on the first transducer, acquire 30 channels for the time it would take until a reflection would have returned (in our case $135 \mu \mathrm{s}$, which is $100 \mathrm{~mm}$ in water), send on the next transducer, wait for the data acquisition, etcetera. In the GUI of our setup this would look like as shown in Figure 8.13, where each 'beam' (horizontal) is firing a transducer, and the white section shows which transducer is fired.

The result would be a matrix of 30 (beams) x 30 (transducers) x 6756 (amount of samples) $\mathrm{x} 2$ (byte) $=12.2 \mathrm{Mbyte}$ per sample, and a total measurement time of $30 \times 135 \mu \mathrm{s}=4.05 \mathrm{~ms}$

Theoretically, this would yield $\left(4.05 \times 10^{-3}[\mathrm{~s}]\right)^{-1}=246[\mathrm{~Hz}]$. What we found out is that the sample rate was limited by the speed of data transmission over the backplane, and the maximum achievable sample rate was ca. $27 \mathrm{~Hz}$.

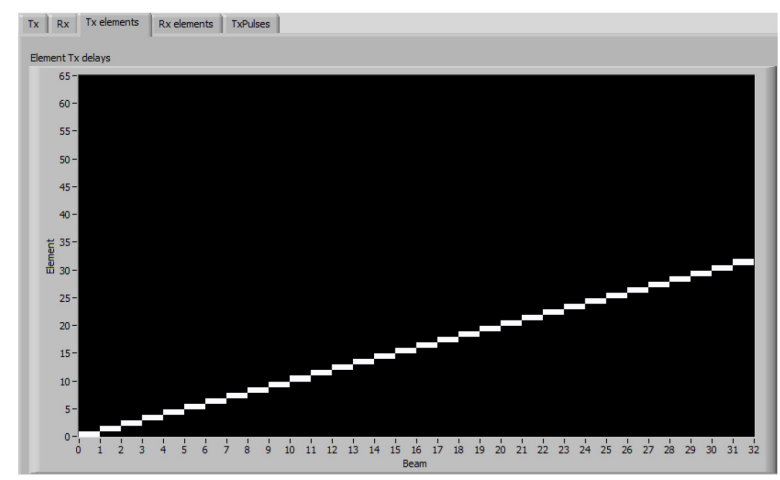

Figure 8.13 This screenshot shows an illustrated table that indicates the order of transmitting pulses for one frame in Full Raw Data mode. Each 'beam' column is a timeslot in which a transducers sends a waveform and the receivers all save data for a predefined period, depending on the maximum depth range. The rows are the 'elements' i.e. transducers. The white cells in 


\section{Supplementary technical details}

the table indicate which transducer is sending in which beam. For FRD, only one transducer is sending every beam, and all transducers are listening. The combination of all beams is called a 'frame', the repetition frequency of these frames determines the frame rate. (here 32 transducers were the initial setting for visualizing FRD, instead of 30)

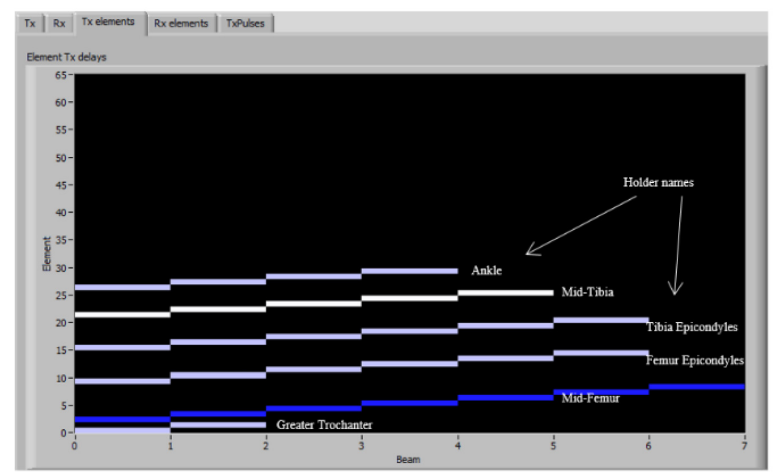

Figure 8.14 This screenshot shows how our setup is configured now. The rows and columns are the same as the figure above, but in this configuration multiple transducers are sending during the same 'beam'. The configuration is made such that in the first beam all first transducers of a holder are transmitting, then in the second beam all second transducers, etcetera. The holder with most sensors determines the number of beams (so in this case the mid-femur holder had seven sensors). Colors are indicating the waveform that is used, see section 8.3.4.

In order to reduce the amount of data, we could use the fact that holders that are lying next to each other do not interfere with each other in the ultrasound measurement; the damping of the tissue is high enough to guarantee this. With the setup as described in Figure 8.13 data is captured by the transducers at the trochanter when the transducers at the ankle are sending. This data cannot be discarded in the FPGA (without rewrite of FPGA code), but we can reorder the transducers in the beams to have a more efficient pattern. This was done by (mis)using the 'Focal Law' feature that was provided by the Diagnostic Sonar application, which allows completely custom configuration of which transducers are transmitting which waveform with how many delay in a beam. In our case it could be used to 'compress' the Full Raw Data per holder:

What can be seen in Figure 8.14 is that each holder gets its own pattern of beams. The order from bottom to top is: trochanter (2 channels), mid-femur(7 channels), femur epicondyle (6transducers), tibia epicondyle (6 transducers), mid-tibia (5 transducers), ankle (4 transducers). 


\section{Supplementary technical details}

In this way the matrix of data becomes 7 beams x 30 transducers, reducing the data size with a factor 4.3. The holder with the largest number of transducers determines the number of beams. This means that also "useless" data (for example beam 2-6 for the Greater Trochanter transducer) is transmitted. Without rewriting the FPGA code, this cannot be prevented in the current setup.

Changing the configuration of the Focal Laws has to be done in a text file. Although the configuration could be derived from the .ini described in 8.5.2, this was not implemented yet, and the user should be sure that the array configuration loaded in the Diagnostic Sonar application matches the configuration of the holders used. The amount of data that had to be streamed from the FPGA to the CPU, and extracting the useful data from the matrix is the key factor that currently limits the acquisition rate of our system.

\subsubsection{Preventing ADC overloading using PulseTables}

Measuring bone reflections in the leg poses a challenge in the dynamic range of the Analog Front End (AFE), which is the analog signal processing path that conditions the signal prior to digitization. Although Time Gain Compensation (TGC) is applied using a Voltage Controlled Amplifier (VCA) prior to digitizing, the setting for the TGC is the same for all input channels. The other setting that is common to all channels is the voltage applied to the three-level pulse. The TGC combined with the common excitation peak voltage does not result in a satisfied common setting to sample all channels in the leg; the transducers that need to acquire bone signal on the thigh need a high voltage and high gains to be able to 'see' the bone beneath several centimeters of tissue, whereas the transducers at mid-tibia are very close to the bone. Without another form of compensation either the signal acquisition of the tibia would be clipped leading to non-linear effects such as clipping and overdrive recovery, severely decreasing the ability to detect bone, or the bone in the thigh would be invisible. 


\section{Supplementary technical details}

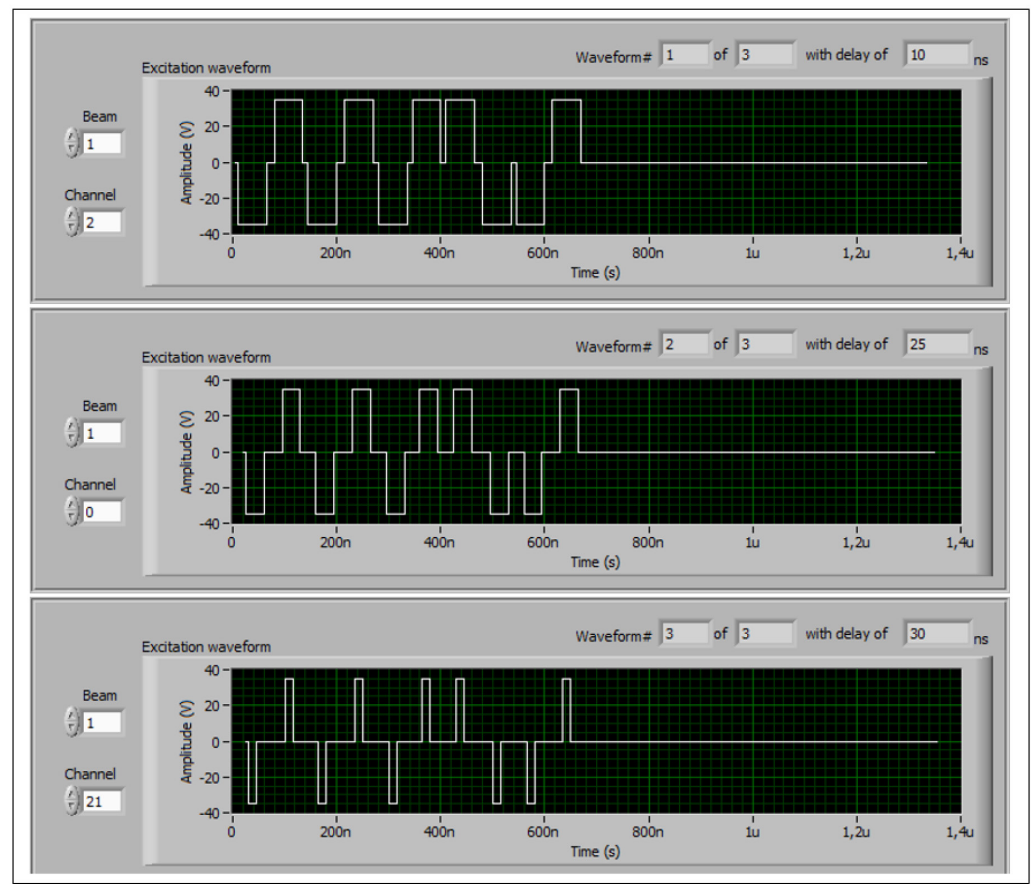

Figure 8.15 The waveforms applied to the three-level pulse. (top): the highest duty cycle; (middle): a lower duty cycle than the highest one; (bottom): the lowest duty cycle.

During the tests with the first setup we had tested a way to change the "duty cycle" of the 3-level pulser, to modulate the amplitude of the output waveform. One of the additional features of the FocalLaw application is that for every beam for every sending transducer a waveform can be programmed. In our case we used that feature to change the duty cycle of the waveform. This is color coded in Figure 8.14, dark blue is the waveform with the highest duty cycle, light blue a lower duty cycle and white is the lowest duty cycle. The individual waveforms are displayed in Figure 8.15. At the thigh a high intensity ultrasound signal is needed because of the large muscles between skin and bone. At the anterior tibia, the tissue layer is very thin, so a low intensity waveform was chosen for that location. These settings were now 'generic' across subjects, but could be personalized based on the expected depth in future research.

\subsection{Ultrasound holder design}

For this thesis, various customized holders were designed to provide a rigid coupling between optical markers and A-mode ultrasound transducers. This rigid coupling can be used to convert the depth found by the transducers combined with the coordinates of the $3 \mathrm{D}$ markers into a point cloud of bone locations. Below, the design of theses holders is described. 


\section{Supplementary technical details}

\subsubsection{Design requirements}

The ultrasound holders are the mediums between the sensors (A-mode ultrasound transducers) and the human subject. Due to the characteristic of ultrasound imaging, the tight skin contact is the primary requirement. Losing skin contact results in useless signal acquisition. Besides, an appropriate pressure on each ultrasound transducer also is necessary to avoid missing skin contact. The configuration of installed A-mode ultrasound transducers and optical markers also need to be taken into account in terms of covering a large anatomical area, while still maintaining line-of-sight between the cameras and the markers during activities. For processing the acquired data, the rigidity of the holders is important.

From a practical point of view, the ultrasound holders should be easy to mount on the subject. Last but not least, the easiness of getting ultrasound signal in high quality on the lower extremity should be assessed systematically in order to screen multiple significantly important anatomical spots in terms of intensity of signal. An experiment was conducted by Mats Boeve in his bachelor assignment [7] by using a ultrasound holder prototype (Figure 8.16) that contained one signal A-mode ultrasound transducer and three optical markers to find the suitable anatomical spots on the lower extremity. The results are shown in Figure 8.17. All designed holders were designed in SolidWorks 2016 (Waltham, Massachusetts, USA). The designed ultrasound holders were manufactured by 3D printing (EOS Formiga P110, EOS GmbH, Krailling, Germany) using Polyamide powder material.

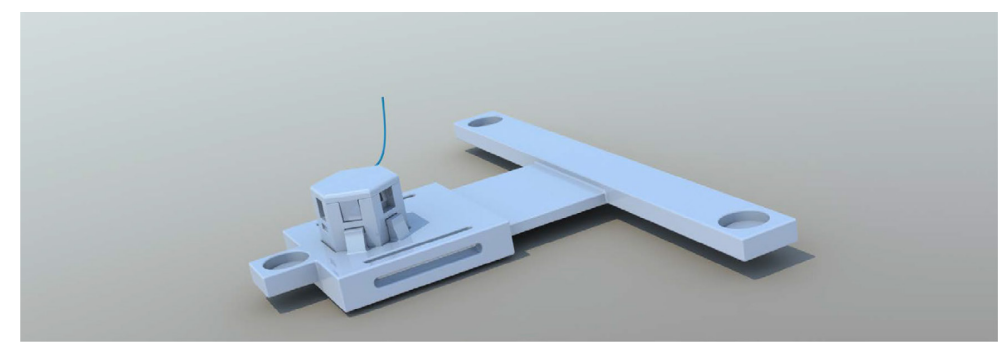

Figure 8.16. The testing ultrasound holder for screening anatomical locations on the lower extremity [7].

\subsection{2 $1^{\text {st }}$ generation}

Based on the screening results of important anatomical locations on the lower extremity, the first generation of ultrasound holders was designed by Mats Boeve during his bachelor assignment. In total, six ultrasound holders were designed to cover six anatomical regions on the lower extremity: great trochanter, middle femur, femoral epicondyles, tibial condyles, middle tibia and ankle. 30 A-mode ultrasound transducers and 18 optical markers were installed in those ultrasound holders. Each holder had three slots for optical markers. The 


\section{Supplementary technical details}

number of A-mode ultrasound transducers on each ultrasound holder ranged from 2 to 7 , as shown in Figure 8.18. The ultrasound holders that have a curved design were parametrized; a protocol was made to measure some dimensions of the subject, so that the holder geometry would be adapted to the size of the individual to optimize skin contact for the specific subject.

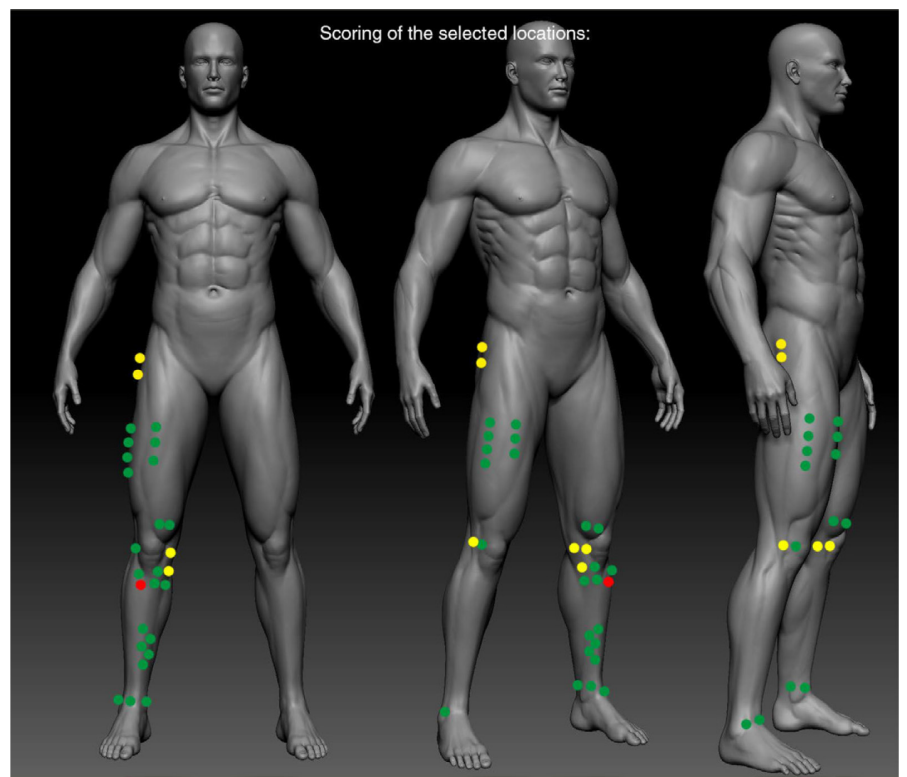

Figure 8.17 The screening results of important anatomical locations on the lower extremity. The easiness of acquiring bony point are labeled in three colors, green (easy), yellow (normal) and red(difficult).

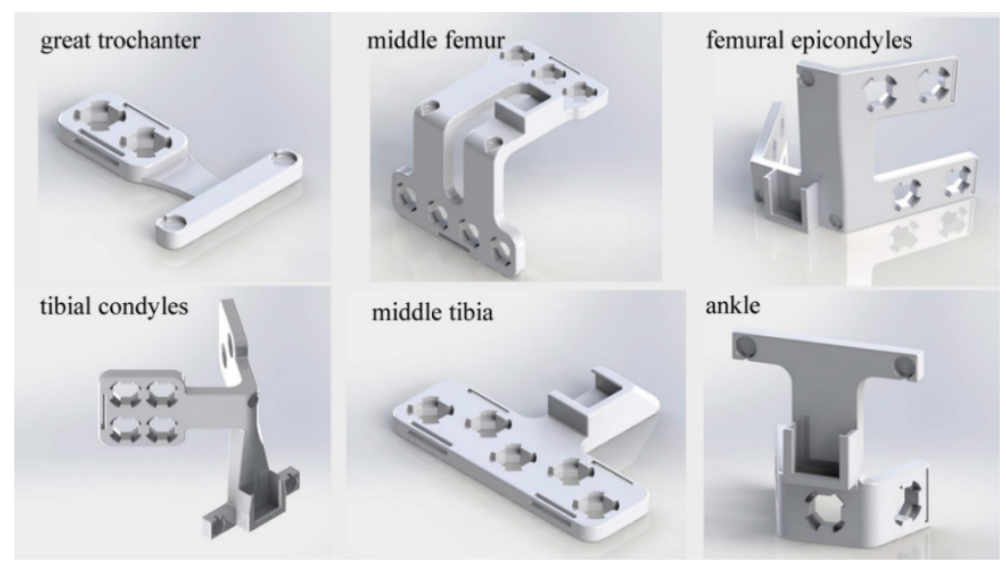

Figure 8.18 The CAD models of designed $1^{\text {st }}$ generation of ultrasound holder.

To test the performance of the design of the $1^{\text {st }}$ generation ultrasound holder, we assessed their performance in a cadaver experiment (Chapter 4). The dimensions of several anatomical 


\section{Supplementary technical details}

regions on the specimen were measured to determine the actual sizes for all ultrasound holders. After adjusting all holders with the dimensions of specimen, the $1^{\text {st }}$ generation holders were 3D printed. The printed ultrasound holders were installed with all 30 A-mode ultrasound transduces and 18 optical markers. During the cadaveric experiment we noticed that some of the transducers were still not making contact with the skin. This was caused by measurement errors in dimensions of the specimen. Since the measurements were performed on a frozen cadaver, size changes occurred in the defrosted condition. As a result of the nonoptimal fit to the cadaver, some anatomical areas could not be measured by the A-mode ultrasound transducers due to loss of skin contact.

\subsection{3 $2^{\text {nd }}$ generation}

As shown above, the $1^{\text {st }}$ generation ultrasound holder had problems maintaining permanent skin contact. To solve this problem, we decided to improve the design and make a $2^{\text {nd }}$ generation holder. The improvement of holder design was implemented by Tomas. A. Johannink as a Capita Selecta Assignment [8]. The femoral epicondyle and tibia condyle areas were separated into two parts covering medial and lateral sides, as shown in Figure 8.19. The middle tibia holder remained the same design as the $1^{\text {st }}$ generation. The length of the greater trochanter holder was extended in vertical direction to improve the stability when attaching it to the subject. The rest of ultrasound holders were redesigned into flat plates. The introduced "optical marker tree structure" (parts in green color in Figure 8.13 where the optical markers are being attached to) increased the flexibility to change the configuration of markers during evaluation. The assessment of this design was conducted on Chapter 6 . The skin contact was significantly improved, compared to the $1^{\text {st }}$ generation.

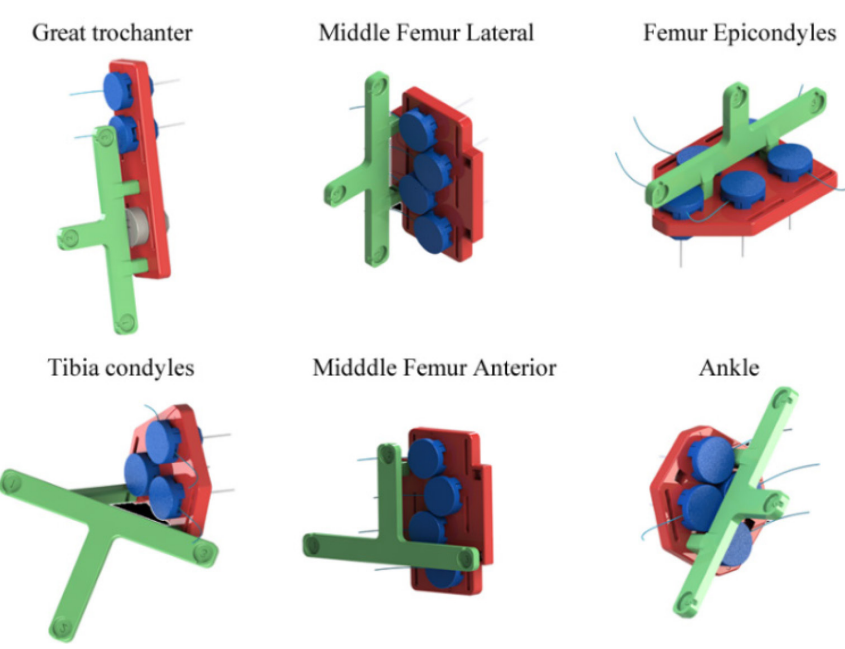

Figure 8.19 The CAD models of the $2^{\text {nd }}$ generation design of the ultrasound holders. 


\subsection{Software architecture}

\subsubsection{Overview}

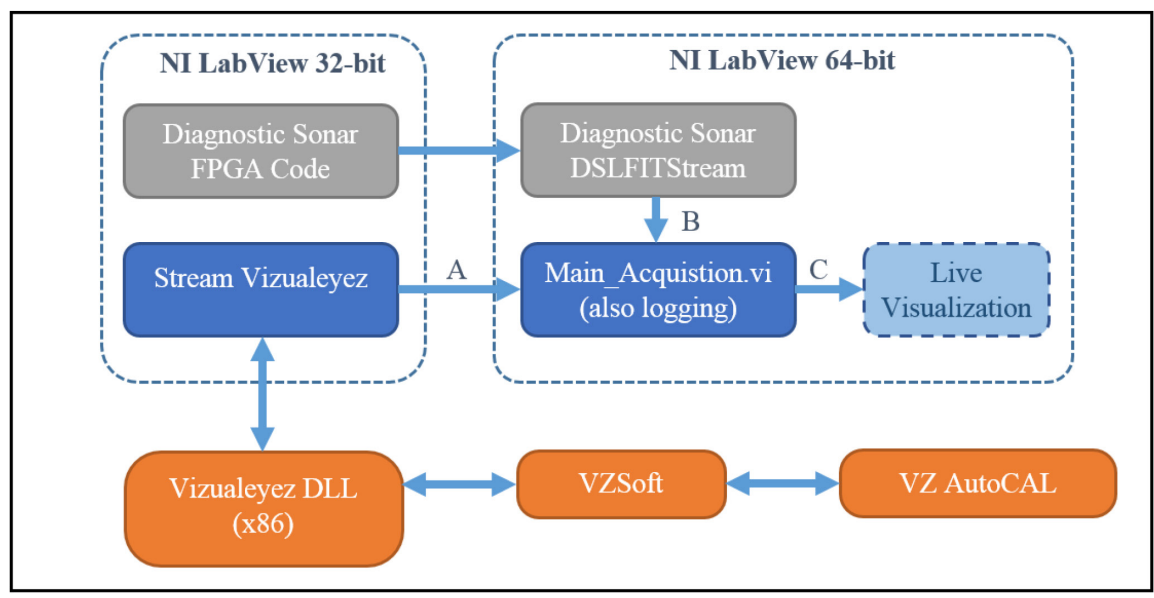

Figure 8.20 A schematic representation of software. Grey boxes are software provided by Diagnostic Sonar, orange boxes are software provided by PTI Phoenix, blue boxes is software developed by University of Twente. Live Visualization is optional. Arrows A is of type 'Network Stream' because this is one of the few possibilities to exchange data between $\mathrm{x} 86$ and 64-bit LabVIEW versions. Arrow B is already a reduced set of scans for one frame, arrow $\mathrm{C}$ is a queue of 'bonetracker' objects, which may be used for visualization.

The main challenges for the software design were the large amounts of data and the integration of data from different sources. The first months that we used the system, all sources for the Diagnostic Sonar application were in LabVIEW 2013, 32-bit. After that we received an update that enabled streaming waveforms from DRAM through the FPGA to the pulsers, which both enabled the Focal Laws feature (8.3.3) and increased the sample rate for both pulsers and ADC's from 40 to $50 \mathrm{MS} / \mathrm{s}$. Another added feature in the same release was that the Diagnostic Sonar application was running in 64-bit LabVIEW, increasing its ability to handle large data sets.

Although these features were very welcome, they did complicate the system integration. Due to the inability of the Xilinx tooling that generates the FPGA code from LabVIEW code to run in 64-bit, the FPGA code was still in 32-bit LabVIEW. This was not much of an issue, because we rarely look into the FPGA code. A larger setback was that by running 64-bit LabVIEW, the interface to the 32-bit Visualeyez DLL was broken. A new interface program was written in 32-bit LabVIEW that reads the samples from the DLL, and sends them over a network stream to the 64-bit code that combines the ultrasound data with the 3D marker data. 


\section{Supplementary technical details}

For visualization, the bonetracker object (see 8.5.2) is put into a queue that can be read by other applications.

\subsubsection{LabVIEW architecture for raw data acquisition}

In the initial setup, and the setup with only one "holder" with one ultrasound transducer (Chapter 2 and 3) the locations of the Visualeyez markers were acquired, data from the ultrasound acquisition was acquired, and this data was combined to find the depth from the tip of the transducer to the bone. When we needed to track 30 transducers in combinations of 2-7 transducers per holder at higher sample rates, we had to come up with a new system to manage the information, as we needed to combine the data per transducer / 3D marker, but also had to maintain the $3 \mathrm{D}$ relation within the holders.

The solution was to use LabVIEW Object Oriented Programming (LVOOP). LVOOP uses the main concepts of object oriented programming, such as classes and objects, inheritance and encapsulation. It should be noted that LVOOP is both a subset and a superset of the features of standard object oriented languages such as Java and $\mathrm{C}++$. It omits advanced features such as multiple inheritance, but adds a LVOOP specific features such as Dynamic Dispatch. More background information can be found on the website of National Instruments[9].

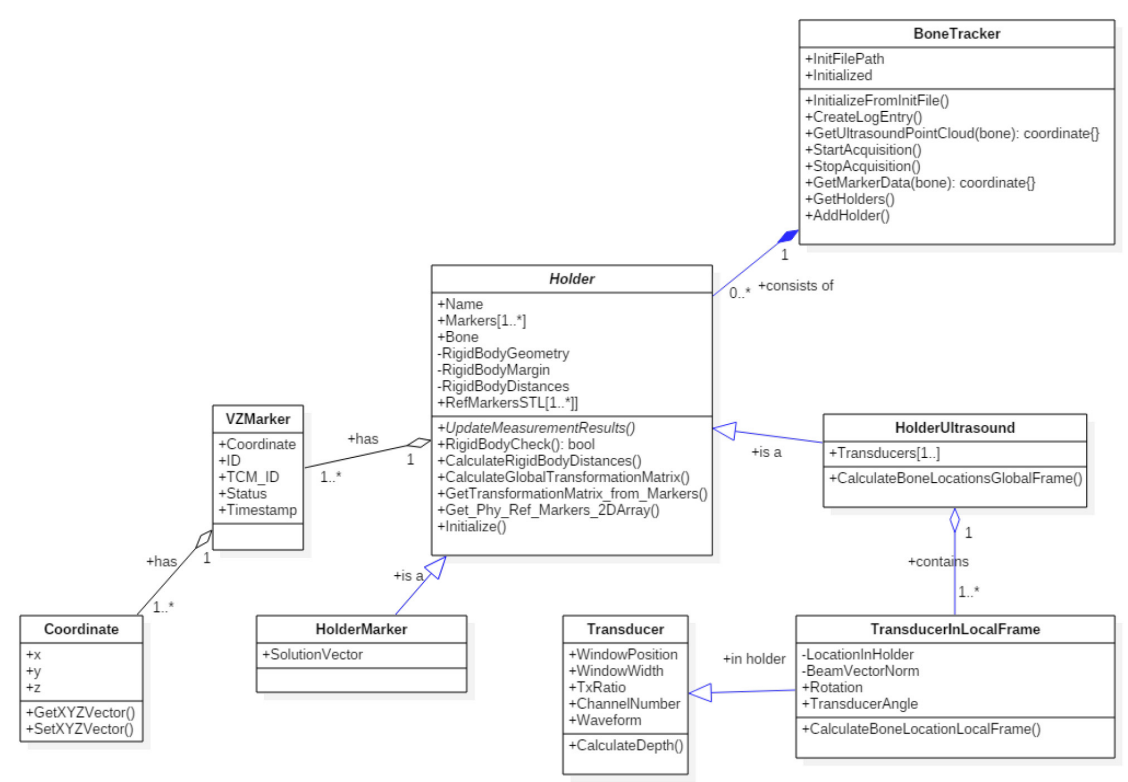

Figure 8.21 The Unified Modeling language diagram of software architecture. 
Our structure is such that we define our setup as the BoneTracker class, which holds reference to both the ultrasound acquisition system as to the Visualeyez 3D marker data. The class is filled with data when a new ultrasound sample has been retrieved from the ultrasound data queue. The BoneTracker class holds several holder classes. In the "holder" class the 3D transformation from the markers to the origin and direction of the ultrasound beam per transducer is known. Each transducer class can calculate the bone depth it has seen.

To know which transducer belongs to which LED in which holder, an .ini file is used to describe these relations. This file is read on startup of the application, and logged with the data to be sure that the post processing algorithms will use the same data as was used during acquisition.

This approach has proven to be very valuable as during the experiments the design of the holders has changed regularly, and both acquisition and post processing code did not need to be rewritten to accommodate for these changes.

The Unified Modeling Language (UML) diagram shows the defined classes in LVOOP (Figure 8.21). BoneTracker Class contains two different kinds Classes derived from Holder Class: HolderMarker Class and HolderUltrasound Class. HolderMarker Class refers to the rigid body attached to the subject only with Visualeyez Markers and without any ultrasound transducer. HolderUltrasound Class refers to the various customized Ultrasound holders mentioned in this thesis, consisting of different numbers of A-mode ultrasound transducers and three Visualeyez wireless markers. The different numbers of A-mode ultrasound transducers are defined in another Class, named TransducerInLocalFrame that describes all necessary information to specify each individual A-mode ultrasound transducer installed in ultrasound holder rigid body, e.g. the local coordinates of each A-mode ultrasound transducer with respect to the origin point local ultrasound holder coordinate system (USelemlocalPts), the unit vector of pointing direction of each transducer (USelemlocalDir). Since each transducer could process its own receiving ultrasound signal and detect the depth of bone, the CalcuationBoneLocationLocalFrame function transforms the detected bone depth to a bony point in the local holder coordinate frame. The member function in HolderUltrasound transforms the calculated local bony points from local ultrasound holder coordinate frame to global world coordinate frame (i.e. Visualeyez camera coordinate frame), in which the transformation matrix is obtained from the corresponding matching from local positions of three Visualeyez markers to the global detecting position of those three Visualeyez markers.

\subsubsection{Pre-processing}

The pre-processing procedure is used to obtain 3D bony points from raw data (received raw ultrasound echo and raw Visualeyez coordinates) through several processes, including 


\section{Supplementary technical details}

correlation, filtering, peak detection and calculation from depth to 3D point. These points are described below.

\subsubsection{Correlation}

The received raw ultrasound echo is correlated with the sending waveform to get the correlated waveform in order to improve the axial sensitivity to sudden impedance changes (such as tissue-bone boundaries). Barker coding was used for correlation as described in Section 8.3.1.

\subsubsection{Filtering}

After correlation, the raw received ultrasound signal is filtered by a second-order low-pass Butterworth filter with a cut-off frequency of $2 \mathrm{MHz}$ (sample rate is $50 \mathrm{MHz}$ ). We used a simple way to filter the correlated waveform. More advanced filtering algorithms could be developed and employed to smoothen the received ultrasound signal and to help to distinguish the reflection referring to bone surface.

\subsubsection{Peak detection}

After filtering, the received ultrasound signal is ready to detect the bone reflection. For each transducer, the reflection that indicates the bone surface is always located within a predictable depth range, discriminating it from those reflections that represent layers of soft tissue. Therefore, we took some simplifications on the peak detection procedure: 1) crop the correlated waveform to get a signal in which we expect to find the reflection of the bone; 2) apply the LabVIEW built-in peak detection method to obtain the peak amplitude and the 2nd time-derivatives of peaks within the cropped sequence of signal. The bone peak is normally sharp when the ultrasound transducer is perpendicular to the bone surface. The 2 nd derivative gives an approximate measure of the sharpness of each peak. Under this context, we assume that the peak with the maximal 2 nd derivatives is the peak referring to the bone surface (above a certain threshold on amplitude).

To extract the bone peak, we estimate the approximate position range based on different anatomical areas where the A-mode ultrasound transducer would be attached. This is a manual procedure that is performed on each subject prior to an experiment. There are two parameters that need to be estimated, termed window position and window width (Figure 8.22). Within the estimated position range, a bone peak could be found and indexed in original received ultrasound waveform to get a time period. 
Supplementary technical details

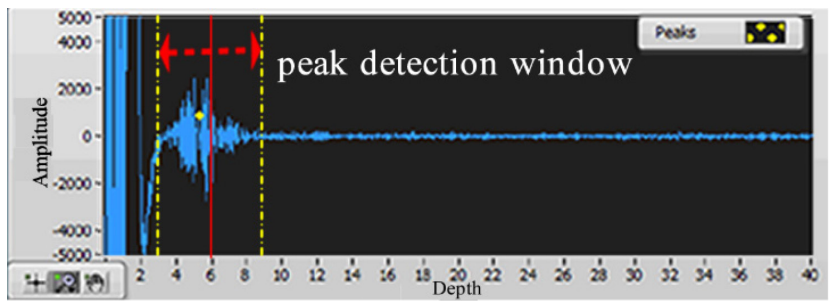

Figure 8.22 An illustration of peak detection window with window width (vertical dashed line in yellow) and window position (vertical solid line in red).

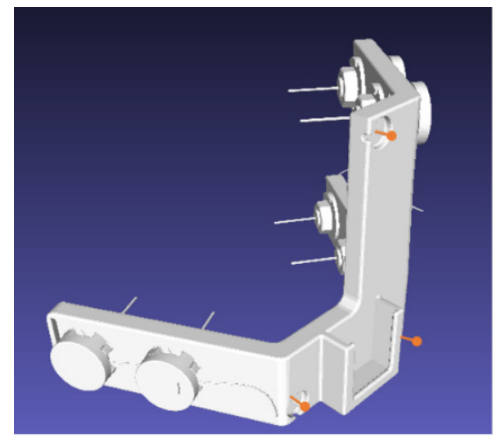

US holder in local coordinate frame

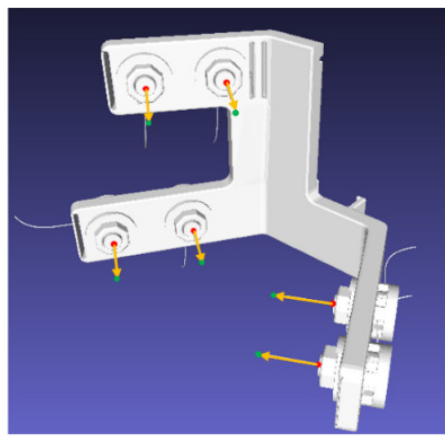

For each US transducer, its beam origin and beam direction in local coordinate frame are known.

Figure 8.23 The example to get the 3D coordinates of ReflectionPointInlocal.

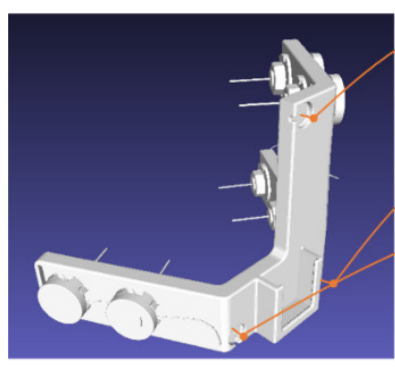

US holder in local coordinate frame

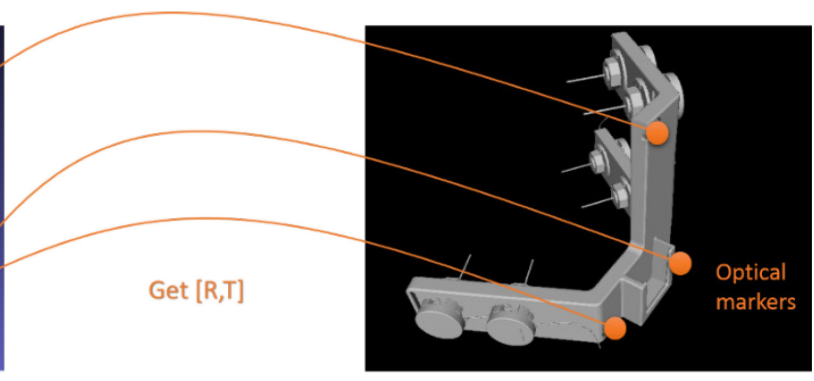

US holder in camera coordinate frame

Figure 8.24 The schematic of the transformation from the local coordinate frame of US holder to the camera coordinate frame.

\subsubsection{Depth to 3D points}

After above calculations, the depth information is obtained from all attached A-mode ultrasound transducers. The A-mode ultrasound transducers are installed in holders with known beam origin (OriginPt) and beam direction (DirectV) of each transducer with respect to the installed optical markers. Therefore, in the local coordinate frame of each 


\section{Supplementary technical details}

ultrasound holder, the depth information can be converted into 3D coordinates (ReflectionPointInlocal) by adding the depth information along the pointing direction for each transducer. Subsequently, the 3D points in local coordinate frame of each ultrasound holder can be obtained and could be expressed in other coordinate frame by applying a transformation $([\mathrm{R}, \mathrm{T}])$, like a $3 \mathrm{D}$ coordinate in optical tracking camera coordinate frame (ReflectionPointInCamera). To demonstrate this calculation, an example is taken by using femoral epicondyles holder of $1^{\text {st }}$ generation design (see Figure 8.23 and 8.24) and the equations are list as below:

$$
\begin{gathered}
\text { ReflectionPointInlocal }(x, y, z)=\text { OriginPt }+(\operatorname{Direct} V \times d) \\
\text { ReflectionPointInCamera }(x, y, z)=\operatorname{ReflectionPointInlocal}(x, y, z) *[\mathrm{R}, \mathrm{T}]
\end{gathered}
$$

\subsubsection{Algorithms for bone pose estimation}

This section describes the algorithms that are used to estimate the $3 \mathrm{D}$ position and orientation of bone segment. The ultrasound holders can produce a discrete point cloud from the bone surfaces. In our case, a point cloud covering the femur and tibia can be obtained after attaching all ultrasound holders onto a subject. The bone shape models of the femur and tibia should be known for our approach to work, in our case the bones were manually segmented from MRI scans.

\subsubsection{Pre-condition}

The bone shape model and the obtained point cloud are the known inputs. In order to estimate the actual bone position and orientation, we need to fit the point cloud with the known bone shape model. It is important to note that the fitting solution should be unique, as the purpose of the fitting is not to find the best shape match between the point cloud and the bone shape model, but to estimate the best pose estimation of the bone shape model that makes sense in the anatomical perspective.

\subsubsection{Pre-registration with weighting}

After clarifying the pre-condition, the pre-registration procedure thus becomes important, since it gives information on the initial guess of pose estimation (for example: femur epicondyles are close to tibia condyles, instead of the hip). However, the obtained 3D bony points from some anatomical areas are not always reliable, because of loss of skin contact and/or missing data of an optical marker. If the pre-registration was implemented based on those areas, then a suitable method is needed to either remove or compensate the instability of those points. In our method, we used a preset weight to compensate this instability. The preset weights are defined empirically based on the geometrical structures. For example, 
femoral epicondyles are difficult to be detected continuously, especially during rapid movements. On the other hand, the points from femoral epicondyles can provide effective geometrical constraints to avoid unrealistic situations. Hence, we set a higher weight for the femoral epicondyle than other areas during pre-registration processing in order to make sure that the $3 \mathrm{D}$ points detected from femoral epicondyle always match to the femoral epicondyle area instead of great trochanter for instance. Finally, the weights are set for all attached ultrasound holder based on the importance of those areas in geometrical constraints (femoral epicondyle both lateral and medial sides: 0.8 , greater trochanter: 1 , middle femur lateral and anterior: 0.2 , lateral tibia condyle: 0.2 , medial tibia condyle: 0.8 , middle tibia: 0.5 , ankle: 0.8 ). These weighting parameters are set empirically, which can be improved in the future.

\subsubsection{Weighted ICP based on each ultrasound signal amplitude}

After applying a weighted pre-registration, the known bone shape model has been transformed roughly to a $3 \mathrm{D}$ position that is close to the obtained point cloud. The next step is to estimate an accurate pose of the bone shape model. This type of task can be solved easily by standard ICP. However, the ICP cannot produce an accurate estimation when the point could has been contaminated by errors (see Chapter 2). To mitigate the influence of unpredictable error, we proposed a modified ICP method with dynamic weighting that is determined by the quality of detected bone peak in received ultrasound signal. It can be regarded as a 'confidence' parameter. As the loss of skin contact results in a low amplitude of the found peak and high amplitude accompanied with a sharp peak indicates the bone surface in a high possibility, the amplitude and the $2^{\text {nd }}$ derivation of the peak provide good parameters for weighting. Since these parameters are changing during measurement, changing weight factors, updated for reliability of the peak detection are applied.

\subsubsection{Data Logging}

Data is logged in National Instruments "TDMS" format, as this can be streamed to disk causing minimal latency in other sections of the program. All settings used for the measurement (the .ini file) are saved in the header of the file, also all ultrasound and 3D marker data is saved per sample. Data is not processed before logging, no bone fitting results or kinematics are saved.

\subsubsection{Optimization for real-time use}

Much effort has been put into generating an application that uses the ultrasound data and processes all the algorithms in soft real-time, rendering a 3D visualization of the STLs of the bones together with transducer locations and projections of the retrieved depth. This 


\section{Supplementary technical details}

visualization has helped in troubleshooting during development and measurements, and could be reused in post-processing.

In order to process all data in real-time at $45 \mathrm{~Hz}$, for-loops were parallelized where possible, and resource-hungry routines were run with debugging features disabled. Where applicable producer-consumer programming structures were used to minimize the effect of variable computation times on the timing of acquisition of ultrasound and optical tracker data.

\section{References}

1. Takashi, A., T. Akihiro, and U. Shin-ichiro, Observation of Ultrasonic Wavefronts by Synchronous Schlieren Imaging. Japanese Journal of Applied Physics, 2002. 41(5S): p. 3308 .

2. Health, C.f.D.a.R. Guidance for Industry and FDA Staff - Information for Manufacturers Seeking Marketing Clearance of Diagnostic Ultrasound Systems and Transducers. $2008 \quad 23 \quad 10 \quad$ 2017]; $\quad$ Available from: https://www.fda.gov/MedicalDevices/ucm070856.htm.

3. Chiao, R.Y. and H. Xiaohui, Coded excitation for diagnostic ultrasound: a system developer's perspective. IEEE Transactions on Ultrasonics, Ferroelectrics, and Frequency Control, 2005. 52(2): p. 160-170.

4. Nowicki, A., et al., Coded ultrasonography. Lecture Notes - ABIOMED, 2004.

5. Lines, D.I. Flexible, high performance ultrasonic array data acquisition and realtime imaging on a PXI platform. Available from: ftp://ftp.ni.com/pub/branches/germany/events/2011/vip/fup/do/structural-physicaltest/02 lines_diagnosticdonar.pdf.

6. Lines, D.I.A., J. Wharrie, and J. Hottenroth, Real-time full matrix capture + total focusing and other novel imaging options using general purpose PC-based array instrumentation. Insight - Non-Destructive Testing and Condition Monitoring, 2012. 54(2): p. 86-90.

7. Boeve, M., Developing a systemof holders for an ultrasound kinematic analysis system.

8. Johannink, T.A., Redesign of A-mode Ultrasound holders for lower body kinematics. 9. Instruments, N. LabVIEW Object-Oriented Programming: The Decisions Behind the Design. 318 2006. 2410 2017]; Available from: http://www.ni.com/whitepaper/3574/en/. 


\section{List of Publications}

\section{Journal contributions}

Kenan Niu, Jasper Homminga, Victor Sluiter, André Sprengers, Nico Verdonschot. Feasibility of A-mode ultrasound based intraoperative registration in computer-aided orthopedic surgery: a simulation and experimental study. Submitted to PLOS ONE.

Kenan Niu, Jasper Homminga, Victor Sluiter, André Sprengers, Nico Verdonschot. Measuring Tibiofemoral Kinematics Using One-channel 3D-Tracked A-Mode Ultrasound Tracking System: A Proof of Principle Study. Submitted to Medical Engineering \& Physics.

Kenan Niu, Victor Sluiter, Jasper Homminga, André Sprengers, Nico Verdonschot. A Novel Multi-channel 3D-Tracked A-Mode Ultrasound System to Measure Tibiofemoral Kinematics. Submitted to IEEE Transactions on Biomedical Engineering.

Kenan Niu, Thomas J Anijs, Victor I Sluiter, Jasper Homminga, André Sprengers, Marco A Marra, Nico Verdonschot. In situ comparison of A-mode ultrasound tracking system and skin-mounted markers for measuring kinematics of the lower extremity. Submitted to Journal of Biomechanics.

Kenan Niu, Victor Sluiter, Jasper Homminga, André Sprengers, Nico Verdonschot. A novel ultrasound tracking system to track in-vivo knee joint motions during walking and stair descent: a feasibility study. Submitted as a book chapter in Intelligent Orthopaedics, Advances in Experimental Medicine and Biology.

\section{Conference contributions}

Kenan Niu, Jasper Homminga, Victor Sluiter, André Sprengers, Nico Verdonschot. The relation between the number of a-mode ultrasound sampled points and the accuracy and robustness registration on computer aided orthopedic surgery. Dutch BME 2015, Egmond aan Zee, The Netherlands. 5th Dutch Bio-Medical Engineering Conference 2015.

Kenan Niu, Jasper Homminga, Victor Sluiter, André Sprengers, Nico Verdonschot. AMode Ultrasound-Based Intraoperative Registration Accuracy Analysis. ESB 2015, Prague, Czech Republic. 21st congress of the European society of biomechanics. European society of biomechanics. 


\section{List of Publications}

Kenan Niu, Jasper Homminga, Victor Sluiter, André Sprengers, Nico Verdonschot. Reconstruction of knee joint kinematics based on 3d-tracked a-mode ultrasound system. Dutch BME 2017, Egmond aan Zee, The Netherlands. 6th Dutch Bio-Medical Engineering Conference 2017.

Kenan Niu, Victor Sluiter, Jasper Homminga, André Sprengers, Nico Verdonschot. A Novel Tibiafemoral Kinematics Measurement System Based on Multi-Channel A-Mode Ultrasound System. CAOS 2017, Aachen, Germany. 17th Annual Meeting of the International Society for Computer Assisted Orthopaedic Surgery.

Kenan Niu, Victor Sluiter, Jasper Homminga, André Sprengers, Nico Verdonschot. A novel tibiofemoral kinematics tracking system based on a-mode ultrasound. ISB 2017, Brisbane, Australia. XXVI Congress of the International Society of Biomechanics. 


\section{Acknowledgement}

Time rewinds to 2013. I graduated after my master study in China and was looking for a $\mathrm{PhD}$ position worldwide, but especially focusing on the vacancies in Netherlands. After having several interviews and sending countless application letters/forms, I still didn't receive any offer at all. I felt very disappointed and depressed at that moment. Just before I decided to give up and look for a company position in China, suddenly, a professor called Nico Verdonschot posted six $\mathrm{PhD}$ positions online (academictransfer.com, thanks this website). He needed people for dynamic MRI imaging, image segmentation, finite element model simulation, musculoskeletal modelling, ultrasound strain measurement and ultrasound based ambulant system. Wow! He wanted to hire lots of people. And that project (BioMechTools) is funded by European Research Council (ERC advanced). Hmmm...never heard that before... whatever! Just applied! In the end, I almost applied all above positions. After two skype interviews, Prof. Nico Verdonschot told me through skype that he would like to offer me this position. At that moment, I almost cannot believe that it was true. After confirming several times, I realized that I got this position. No word can describe my feeling at that moment. Shocked? Surprised? Happy? Panic? Joyful? Or a mixture of above all.

Back to now, the four years' journey of my $\mathrm{PhD}$ study results in the work presented in this thesis, which is an absolutely valuable and memorable experience. It would not be possible to finish this thesis without the contributions from many people, to whom I would like to express my sincere gratitude here.

First of all, I would like to thank my supervisor Nico Verdonschot and co-supervisors Jasper Homminga and André Sprengers for giving me this opportunity to do my $\mathrm{PhD}$ research in BW group at the University of Twente. Nico, thanks for your guidance for my research and efforts in correcting my papers and thesis. I will always be impressed by all your critical questions from a clinical perspective which have strongly inspired me to improve the integrity, precision and clarity of the research. I appreciate your sense of humor during the meeting. Although most of time they turned to cold jokes, they alleviated atmosphere of tension from the meeting. Sometimes, the pressure of research became to the bets between you and me. In the end, I still don't know if Victor and I win a champagne or you get a good wine (as we have reconstructed the motion of jumping down). Jasper, thanks for your invaluable support, great coordination, and thoughtful guidance for my $\mathrm{PhD}$ study in all these years. I have learned a lot from you with respect to the attitude of scientific research, and expression and writing skills. Your rational suggestion always points out a clear way to me through the darkness. The story about the pencil and complex ball-pen for the astronaut during the cold war not only continuously reminded me of thinking outside the box during 


\section{Acknowledgement}

my $\mathrm{PhD}$ study, but also will guide me in the future. You acted as lubricants in our team and this smoothed the intensive discussions during the regular ERC meeting every Thursday. André, thanks for your encouragement, knowledges on the mathematics and physics (also on the movies, music, cultures and beers), optimistic attitude and huge support during my entire $\mathrm{PhD}$ study. You are always full of passion and creativeness and this special "aura" encouraged me to look to the front and keep confident. "Everything's Gonna Be Alright".

Secondly, I want to express my great gratitude to Victor, as my colleague, my mentor, my Jedi master, my friend and one of my paranymphs. I really appreciate your cooperation, your professional skills, your patience and efforts on our research. Without your significant contributions, this thesis would not have been completed and this technique would not have been realized so quickly. You also gave great helps in my "cooking skills" in LabVIEW. Whereas, I always made "spaghetti" and you seemingly didn't like it. It was my honor to work with you during my $\mathrm{PhD}$ study and I really appreciate your efforts and guidance in my research and your helps in my daily life in Enschede, such as taxes, car driving, car maintenance, moving and all translation and explanation about Dutch culture and rules. Thanks again to Linda and you for providing such a nice place for the Hot-pot night. It was wonderful and I will remember it always. Next time, I prefer to drink a bottle of Oude Jenever rather than a glass of Er-guo-tou.

I wish to express my gratitude to all the committee members Prof. dr. ir. P.H. Veltink, Prof. dr. S. Manohar, Prof. dr. ir. G. J. Strijkers, Prof. dr. B.M. ter Haar Romeny, Dr. M.M. van de Krogt, Dr. Ing. S. van de Groes for spending time to read my thesis, for allowing me to give defense, and for taking part in the defense ceremony.

I spent unforgettable four years at the BW group as a $\mathrm{PhD}$ student. First of all, I would like to thank Lianne, so kind and friendly, always available to help me since my first working day till the last days before my defense. As I remembered, someone told me that Lianne would be more important than your supervisor for your $\mathrm{PhD}$ when I just started in the BW group. That is totally true. Same gratitude to Jeanine, although you mixed Hao's name and mine sometime, you always provide effective help in many things from reimbursements to daily support. I felt lucky that we have you and Lianne as our secretaries in BW group. I would also like to thank Bart, as the big boss of our group. Our offices were opposite before the group moved to the ground floor. At that moment, I felt a bit of nervous that you were able to monitor my attendance, but you never criticized that I was late for the work. Nikolai, thanks for all your IT supports during my PhD. I may bother you with all kinds of simple, strange and weird IT problems, from software license to reinstall windows and upgrade hardware and laboratory usages. Although you were quite busy usually and I needed to 
remind you several times, the problems always can be solved completely if you were available. Wouter, thanks for your supports from the mechanical manufacture and sharing your experience on drone webcam. Hope you will have no problem in reading Chinese manual when you buy new stuffs from Alibaba.

Special thanks to Hao for being my paranymph. Hao, we have acquaint with each since our bachelor study. We also stayed at same university for master. Subsequently, also for the $\mathrm{PhD}$. We have shared many memorable moments. We have many interests in common, such as playing basketball, gym, and watching Dota Ti and playing wang-zhe-rong-yao (though Chi-ji is more popular now, winner winner! chicken dinner). All the adventures we had still remain in my mind: Diamond trip in Amsterdam, car sick trip for looking for Chinese supermarket in Nijmegen, and of course all the dinners and parties. I am grateful to have you as my colleague, my officemate, my classmate and one of my best friends. Wish you can finish you $\mathrm{PhD}$ as soon as possible.

I am fortunate to have so many lovely colleagues in such a wonderful group. Thanks for your helps in my PhD study. Here I express my gratitude to the BW members: Vincenzo, René, Letian, Steven, Iris, Riza, Hao, Corine, Han, Hielke, Vera, Mark, Heidi, Amber, Niek, Simone, Bob, Kostas, Stergios. Thank you all once again for giving me such a nice time in the group. I also would like to express my gratitude to Tomas and Mats. You all contributed a lot to my research, though you spent only short time in the group. Without your help, the work could not have been done so much. I wish you all have a bright future.

I would also like to express my gratitude to all BioMechTools members and colleagues in Nijmegen. Macro, thanks for your explanation with patience for all biomechanical modelling knowledge during my $\mathrm{PhD}$. Hamid, thanks for your helps and cooperation during my first cadaver experiment. Valentina, thanks for scanning MRI image for all participants for my research. Kaj, thanks for sharing your ultrasound knowledge with me and your advices in my experiments. Dennis, thanks for your coordination between the cadaver experiment and radiology department. Richard and Léon, thanks for your generous helps in all cadaver experiments and image segmentation. Thomas, thanks for your helps in the comparison experiments and the detailed discussion on the paper writing.

I am also grateful to have so many wonderful Chinese friends in Enschede, Many thanks to Lantian, Duying, Wangrong, Xiaolin, Weiye, Tao Yin, Zhan Luman, Fangzhou, Tianpei, Letian, Jingwei, Zhonghua, Leilei, Xiaobin, Xingwu, Junwen, Yeliang, Guo zhengchao, Liangjia, Mengdi, Youwen, Hairong, Aijie, Wang Yi, Huanghe, Wenlong, Jiahui, Liuyao, Yanghuan, Tian lulu, Yuxi, Duliang and all others. Also best wishes and many thanks for 


\section{Acknowledgement}

previous BMIE-Dutch friends, Huangfan, Xiaocai, Fenghua. I am glad to have you as my friends. It was great to have you together for all parties, trips, dinners and laughs and joys.

Finally, I deeply thank my family for their great support. Without you I could not reach this point of my life. I would like to thank my parents who give me the motivation to start and finish my $\mathrm{PhD}$. My special thanks to my grandparents who taught me a lot of life experience and life creeds that broaden the view of life. Xiaohua, I am grateful and happy to have you in my life. You made my life and myself better. Thanks for your great support and supervision during my $\mathrm{PhD}$. We have experienced so many things together so that I would like to spend my life with you for explore the unknown, amazing and beautiful world. Thank you for everything. I believe our life will be better and better.

Kenan Niu

January, 2018 


\section{About the author}

Kenan Niu was born on 18th December 1988, in Laiwu, Shangdong Province, China. He has obtained a Master degree (2013) and a Bachelor degree (2011) on biomedical engineering from Biomedical and Information Engineering School, Northeastern University, Shenyang, China. He worked on the topics "The Research Based On The Optimal Path Planning And Image Navigation Of Lung Biopsy Surgery" and "Research on Interactive Volume Exploration System By Means of Tablet PC and Real-Time 3D Position Tracking Device" for his master and bachelor theses respectively, under the supervision of Prof. Yan Kang. Afterwards, he carried out his PhD on an ERC advanced grant of "BioMechTools" under the supervision of Prof. Nico Verdonschot at Department of Biomechanical Engineering of the University of Twente, Enschede, The Netherlands. 
ISBN: $978-90-365-4482-5$

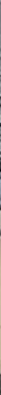

